\title{
Alternative Methods of Bioactive Compounds and Oils Extraction from Berry Fruit By-Products-A Review
}

\author{
Iga Piasecka $^{1, *(\mathbb{D}}$, Artur Wiktor ${ }^{2} \mathbb{D}$ and Agata Górska ${ }^{1} \mathbb{D}$ \\ 1 Department of Chemistry, Institute of Food Sciences, Warsaw University of Life Sciences, \\ 159c Nowourysynowska Street, 02-776 Warsaw, Poland; agata_gorska@sggw.edu.pl \\ 2 Department of Food Engineering and Process Management, Institute of Food Sciences, Warsaw University of \\ Life Sciences, 159c Nowoursynowska Street, 02-776 Warsaw, Poland; artur_wiktor@sggw.edu.pl \\ * Correspondence: iga_piasecka@sggw.edu.pl; Tel.: +48-22-5937607
}

check for updates

Citation: Piasecka, I.; Wiktor, A.; Górska, A. Alternative Methods of Bioactive Compounds and Oils Extraction from Berry Fruit By-Products-A Review. Appl. Sci. 2022, 12, 1734. https://doi.org/ 10.3390/app12031734

Academic Editor: Dino Musmarra

Received: 15 January 2022

Accepted: 6 February 2022

Published: 8 February 2022

Publisher's Note: MDPI stays neutral with regard to jurisdictional claims in published maps and institutional affiliations.

Copyright: (C) 2022 by the authors. Licensee MDPI, Basel, Switzerland. This article is an open access article distributed under the terms and conditions of the Creative Commons Attribution (CC BY) license (https:// creativecommons.org/licenses/by/ $4.0 /)$.
Abstract: Berry fruit by-products are a source of polyphenol compounds and highly nutritious oils and can be reused to fulfill the requirements of the circular economy model. One of the methods of obtaining polyphenol-rich extracts or oils is extraction. Applying conventional solvent extraction techniques may be insufficient to reach high polyphenol or lipid fraction yields and selectivity of specific compounds. Alternative extraction methods, mainly ultrasound-assisted extraction, pulsed electric field-assisted extraction, microwave-assisted extraction and supercritical fluid extraction, are ways to improve the efficiency of the isolation of bioactive compounds or oils from berry fruit by-products. Additionally, non-conventional techniques are considered as green extraction methods, as they consume less energy, solvent volume and time. The aim of this review is to summarize the studies on alternative extraction methods and their relationship to the composition of extracts or oils obtained from berry waste products.

Keywords: berry fruit by-products; alternative extraction methods; waste management; green extraction; PEF-assisted extraction; ultrasound-assisted extraction

\section{Introduction}

From a botanical point of view, berry fruit is an artificial fruit classification. However, it is the term that is commonly used to refer to the the group of fruits from Rubus (raspberry, blackberry), Ribes (currants, gooseberry), Aronia (chokeberry), Vaccinium (cranberry, blueberry) and Fragaria (strawberry) genera. According to FAO statistics, berry fruit production reaches an amount of over 12.2 million tons worldwide [1]. The latest data, including area and quantity of production, is presented in Figure 1.

Berries, as well as other fruits, may be consumed raw or can be processed to such products as, e.g., frozen, dried or canned fruits, juices. Those products may be further processed as well [2]. However, every step of processing and transport may generate losses reaching, according to the $\mathrm{FAO}$, even $45 \%$ of fruits and vegetables produced [3]. Such significant percentages of loss and waste not only have economic consequences, but also affect the natural environment, especially water use [4]. In order to obtain economic and environmental benefits it is widely recommended to apply circular economy model concepts in the food production chain. The model includes the further use of by-products as a way of managing and minimizing production of wastes when they are still a source of bioactive, highly nutritive compounds [5]. Berries are mainly processed to juices and concentrates. The technological scheme of juice production leads to pomace generation-a major fruit processing by-product, which contains stem cells, skins and seeds of fruits. The aim of this review is to present and systematize possible methods of bioactive compounds and oils extraction from chosen berry fruit pomaces and the impact of certain extraction methods on the quality and composition of those extracts. 


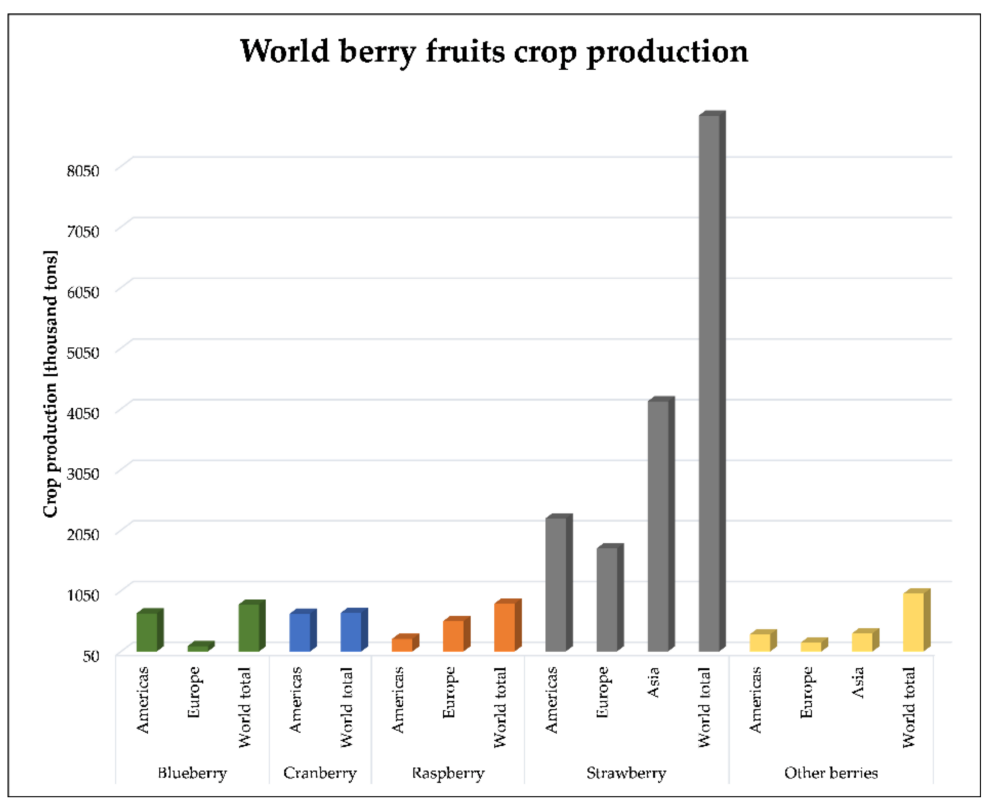

Figure 1. World berry fruits crop production (2019).

\section{Composition of Berry Pomaces}

\subsection{General Information}

Contents of pomaces depend on the berry species, but as they are rich in the seeds and skins of fruits, dietary fiber is a dominant component of pomaces. For instance, chokeberry pomace powder consists of ca. 3.61\% of fat, $5.97 \%$ of protein, $28.8 \%$ of carbohydrates and $57.8 \%$ [6] to 59.5\% [7] of fiber (mostly insoluble fraction). McDougall and Beames [8] studied the composition of raspberry pomace and the composition of the studied by-product was described as follows: $11.1 \%$ of fat, $10.0 \%$ of protein and $59.5 \%$ dietary fiber. In a different study conducted by Górnaś et al. [9], the concentrations of constituents in raspberry pomace were: $9.1 \%, 8.7 \%$ and $54.2 \%$ for fat, protein and dietary fiber, respectively. In the same study, the composition of strawberry and blackcurrant pomaces was determined. In strawberry pomace, concentrations of nutrients were at levels 3.4\%, 9.2\% and 33.9\% and in blackcurrant pomace at levels $0.7 \%, 6.9 \%$ and $38.5 \%$ for fat, protein and dietary fiber, respectively. Based on the studies conducted by Reißner et al. [7], it can be stated that the physicochemical properties of currant pomace depend on the color group of the berry. Blackcurrant (Ribes nigrum) pomace powder was found to contain about $61.0 \%$ of seeds. The percentage contents of the nutritional components of blackcurrant pomace were as follows: $20.21 \%$ fat, $15.71 \%$ protein, $2.20 \%$ carbohydrates and $59.13 \%$ fiber, with a predominance of insoluble fiber. Redcurrant (Ribes rubrum) pomace consisted of $40.4 \%$ seeds and, based on the obtained results, it can be treated as a source of $14.23 \%$ fat, $11.76 \%$ protein, $12.65 \%$ carbohydrates and $58.1 \%$ fiber, mostly insoluble fiber. Gooseberry pomace powder in turn consisted of $34.2 \%$ seeds, $10.93 \%$ fat, $12.40 \%$ protein and $56.6 \%$ dietary fiber, over $87 \%$ of which was an insoluble fraction.

\subsection{Polyphenols}

\subsubsection{Role of Polyphenols}

Polyphenols belong to a group of secondary metabolites present in plant-derived food. They are the most common antioxidants in the human diet and consist of different compounds; however, their classification is not strict. Generally, the term 'polyphenols' refers to flavonoids (with the subgroups: anthocyanins, flavanols, flavanones, flavones, flavonols and isoflavonoids), tannins, stilbenes and phenolic acids and their derivatives [10].

Although polyphenols are considered as non-nutritive compounds, they play a role in disease prevention and help to improve health due to their ability to neutralize free radicals [11]. There are a number of results of meta-analyses available concerning the influence 
of polyphenols on human functions. They may have potential in improving performance in groups of healthy humans [12], stimulating the growth of health-promoting species but inhibiting development of pathogenic organisms in gut microbiota [13]. Supplementation of polyphenolic compounds can be helpful in inflammatory bowel disease therapy [14], brain functions [15], lipid profile and inflammation status [16] improvement. In addition, an association between anthocyanins intake and lowered risk of hypertension [17] and cancer [18] was found.

\subsubsection{Bioavailability of Polyphenols}

However, there are limitations related to the bioavailability of some polyphenols and their stability during processing or storage. There are several factors that may affect the stability of phenolic compounds, for instance, $\mathrm{pH}$, temperature, interactions with other food components, access to light and oxygen and metal ions presence and abundance [19]. Polyphenols tend to be more stable in acidic than in alkaline conditions; also, storing or processing foods at high temperatures leads to decreases in polyphenol content [20-22]. There are some studies which indicate that increased temperature may result in the appearance of other polyphenols in heated material as compared to unheated samples. This might be an effect of polymerization or polyphenol release from certain components [23].

\subsubsection{Polyphenol Content in Berry Pomaces and Their Antioxidant Activity}

Industrial chokeberry pomace consists of a solid number of bioactive compounds. Total polyphenol content (TPC) of chokeberry pomace reaches $5.5 \mathrm{~g} / 100 \mathrm{~g} \mathrm{dm}$ (dry mass), expressed as catechin monohydrate, determined using the Folin-Ciocalteu method. The main groups of polyphenols were found to be anthocyanins at $1.80 \mathrm{~g} / 100 \mathrm{~g} \mathrm{dm}$, followed by phenolic acids at $0.31 \mathrm{~g} / 100 \mathrm{~g} \mathrm{dm}$ and flavonols at $0.184 \mathrm{~g} / 100 \mathrm{~g} \mathrm{dm}$. AA (antioxidant activity) was measured as $1111 \mu \mathrm{mol} \mathrm{FE} / \mathrm{g}$ in the ferric reducing antioxidant power (FRAP) assay [6]. Consistent results were obtained in a composition analysis of a storebought product which contained $100 \%$ chokeberry pomace. TPC measured using the Folin-Ciocalteu method reached $4233 \mathrm{mg}$ GAE/100 $\mathrm{g} \mathrm{dm}$ and anthocyanins content determined by the $\mathrm{pH}$ differential method was $1165 \mathrm{mg}$ CGE/100 $\mathrm{g} \mathrm{dm}$. AA, measured in a FRAP assay amounted to $47.38 \mathrm{mmol} \mathrm{FE} / 100 \mathrm{~g} \mathrm{dm}$ and was measured in a DPPH assay as $131.06 \mathrm{mmol} \mathrm{TE} / 100 \mathrm{~g} \mathrm{dm}$ [24]. The main phenolic compounds in chokeberry pomace, detected using HPLC, were polymeric procyanidins with a concentration of $9586 \mathrm{mg} / 100 \mathrm{~g} \mathrm{dm}$, which was two-fold higher than the result for fresh berries and almost seven-fold higher than that for juice [25].

The TPC of industrially obtained seedless blackcurrant pomace, measured using the Folin-Ciocalteu method, was in a range of 1855.5-2241.6 mg EE/100 g pomace. Specific polyphenol composition determined using HPLC indicated that anthocyanins are the dominant components, reaching values of 344.6-1046.1 mg/100 g pomace and depending on the year of fruit harvest. AA values determined in a DPPH assay ranged from 93.3$126.5 \mu \mathrm{mol} \mathrm{TE} / \mathrm{g}$ pomace [26]. A comprehensive study of redcurrant, raspberry and blackberry pomaces conducted by Jara-Palacios et al. [27] showed that redcurrant pomace was characterized by the highest values of TPC, as determined by the Folin-Ciocolteau method, which were equal to $3446.59 \mathrm{mg}$ GAE/100 $\mathrm{g} \mathrm{dm}$, followed by $2014.66 \mathrm{mg}$ GAE/100 $\mathrm{g} \mathrm{dm}$ for raspberry pomace and $1699.62 \mathrm{mg} \mathrm{GAE} / 100 \mathrm{~g} \mathrm{dm}$ for blackberry pomace. Anthocyanin concentrations, determined in a HPLC/MS analysis, were similar for all pomaces and ranged from $149.91 \mathrm{mg} / 100 \mathrm{~g} \mathrm{dm}$ for redcurrant pomace to $188.05 \mathrm{mg} / 100 \mathrm{~g} \mathrm{dm}$ for raspberry pomace. As AA was correlated with TPC, redcurrant was characterized by the highest AA (tested using ABTS), $60.83 \mathrm{mmol} \mathrm{TE} / 100 \mathrm{~g} \mathrm{dm}$, while lower AA values for raspberry and blackberry were observed, these being, respectively, 29.75 and $22.54 \mathrm{mmol} \mathrm{TE} / 100 \mathrm{~g} \mathrm{dm}$.

TPC, as determined by the Folin-Ciocalteu method, in blackberry pomace extract obtained from wild fruits ranged from $48.28-50.16 \mathrm{mg} \mathrm{GAE} / \mathrm{g} \mathrm{dm}$ and from cultivated fruits ranged from 26.30-35.40 mg GAE/g dm, which indicates that phenolic compound concentrations are higher among wild blackberries [28]. The phenolic composition of raspberry pomace was described. TPC, measured using HPLC, was determined as $238.36 \mathrm{mg} / 100 \mathrm{~g} \mathrm{dm}$. 
Anthocyanins were the dominant phenolic compounds, reaching nearly $83 \%$ of the TPC, followed by ellagic acid and flavanols [29].

\subsubsection{Applications of Polyphenolic Extracts}

As berry pomaces are rich in polyphenols, they may yield polyphenol-rich extracts. The use of extracts obtained from berry pomaces is gaining the interest of researchers. There are papers reporting applying chokeberry pomace extract to enrich the composition of apple juice. Fortified products represent increased acidity, levels of vitamin C, TPC, total flavonoids, total anthocyanins and higher AA, as determined by the ABTS method [30]. Raspberry pomace extract can be recognized as an antioxidative but also as an antibacterial ingredient [31]. Extracts from chokeberry pomace were considered for use as an ingredient of chitosan-based packaging films. Adding the extract in film formulation resulted in its decreased solubility and has a possible application as a $\mathrm{pH}$-indicating film due to the high stability in acidic conditions of anthocyanins [32]. Berry pomace extracts may also be applied as natural, antioxidant colorants [27].

\subsection{Lipid Fraction}

As pomaces contain seeds, they are a source of lipophilic components. Oils obtained from berry by-products using traditional methods (solid-liquid extraction, maceration, coldpressing) vary in composition and concentration of fatty acids, phospholipids, tocopherols, sterols and other bioactive compounds, e.g., carotenoids. They may also have different oxidative and thermal stabilities or shelf lives. This variation is caused by species differences in fruits [33], fruit growing conditions [34] and conditions of storage of material and oil [35].

\subsubsection{Fatty Acid Composition}

Fatty acid profiles may differ significantly even in the same genus of a berry plant. The results of the research conducted by Šavikin et al. [36] on Ribes sp. show variation of specific FAs depending on the color of fruit, with blackcurrant reaching the highest values of LA and GLA, but the lowest for ALA and OA. Red- and white currant presented similar concentrations of LA, GLA and ALA, whereas the white type was characterized by the highest values of OA. Results showing SFA content were not diverse. Table 1 presents the fatty acid profiles of berry oils. According to this summary, it can be concluded that the considered berry seed oils are rich in unsaturated, mostly polyunsaturated (PUFA), fatty acids. However, they differ in terms of specific fatty acid profiles. The dominant fatty acid in berry seed oils is linoleic acid (C18:2, n6); its content ranges from $33.86 \%$ for gooseberry [37] to $71.1 \%$ for chokeberry oil [38]. Additionally, the content of linolenic acids is high, except for chokeberry oil. The composition of fatty acids results in oil properties. High PUFA contents (especially linoleic and $\alpha$-linolenic acids) lower the stability of plant oils, so they are more susceptible to oxidation and are characterized by shorter shelf lives [39-41]. Moreover, a high amount of MUFAs results in reduced stability values, although to a lesser degree than with PUFAs [42]. However, the nutritional value of unsaturated fatty acids is significant. FAO/WHO recommends replacing intake of saturated fatty acids with unsaturated fatty acids, especially PUFAs [43]. Numerous meta-analyses and review papers describe the positive impact on human health of marine-derived PUFAs, EPA and DHA [44-47], although plant-derived PUFAs can be elongated and desaturated into AA, EPA or DHA in the human system [48]. In addition, some reports claim that all-sourcederived PUFA intake reduced all-cause mortality [49], while $n 3$ PUFA intake reduced the risk of metabolic syndrome [50]. Plant-derived PUFAs' ability to regulate serum insulin levels has been described [51]. The most common FAs in berry oils, linoleic acid and $\alpha$-linolenic acid, are classified as essential fatty acids and have to be delivered by food consumption due to the human disability for their endogenic production [52]. MUFAs can be produced in the human organism [53], but food-derived MUFAs were also found to have a role in disease prevention, especially in glucose-insulin management and reduced risk of co-existing diseases, in a group of diabetic patients [54-56]. 
Table 1. Fatty acid profiles (\%) of oils extracted from berry pomaces. (C16:0—palmitic acid, C16:1—palmitoleic acid, C18:0—stearic acid, C18:1—oleic acid, C18:2—linoleic acid, C18:3—linolenic acid, C18:4—stearidonic acid, C20:0—arachidic acid, C20:1—paullinic acid, C20:2—eicosadienoic acid, C22:0—behenic acid.)

\begin{tabular}{|c|c|c|c|c|c|c|c|c|c|c|c|c|}
\hline $\begin{array}{c}\text { Source of } \\
\text { Oil }\end{array}$ & C16:0 & C16:1 & C18:0 & C18:1 & C18:2 & C18:3 & C18:4 & C20:0 & C20:1 & C20:2 & C22:0 & Reference \\
\hline Chokeberry & $5.1-7.22$ & $0.15-0.53$ & $1.1-1.39$ & $\begin{array}{c}n 9 \text { cis: } 23.47 \\
n 9 \text { trans: } 0.93 \text { or total: } \\
17.48-21.4\end{array}$ & $64.67-71.1$ & $\begin{array}{l}n 3: 0.34-0.92 \text { or } \\
\quad \text { total: } 0.5\end{array}$ & - & $0.6-0.81$ & 0.25 & 5.26 & $0.38-0.8$ & {$[38,57,58]$} \\
\hline Raspberry & $2.43-2.92$ & $0.08-0.12$ & $0.87-1.45$ & 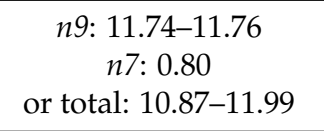 & $51.44-54.52$ & $\begin{array}{c}n 3: 6.68-31.68 \\
n 6: 0.07 \\
\text { or total: } 29.11\end{array}$ & - & $0.37-0.62$ & $0.13-0.14$ & $0.03-0.33$ & $0.10-0.34$ & {$[37,38,58-60]$} \\
\hline Blackberry & $3.47-4.52$ & $0.03-0.13$ & $2.10-2.87$ & $\begin{array}{c}n 9: 7.50-12.17 \\
n 7: 0.56 \\
\text { or total: } 14.72\end{array}$ & $61.22-67.96$ & 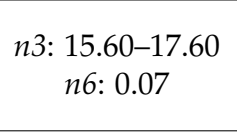 & - & $0.47-1.06$ & $0.31-0.38$ & 0.15 & $0.12-0.16$ & {$[37,58,59]$} \\
\hline Blackcurrant & $4.49-6.5$ & $0.03-0.1$ & $1.4-1.93$ & $\begin{array}{c}n 9: 10.2-13.79 \\
n 7: 0.35-0.7 \\
\text { or total: } 16.1\end{array}$ & $41.41-57.8$ & $\begin{array}{l}\text { n3: } 12.91-14.9 \\
n 6: 13.9-15.6 \text { or } \\
\text { total: } 13.2\end{array}$ & $2.7-3.89$ & $0.04-0.2$ & $0.16-1.0$ & $0.06-0.3$ & $0.1-4.7$ & {$[38,59,61,62]$} \\
\hline Redcurrant & $4.8-6.88$ & 0.09 & $1.29-3.0$ & $\begin{array}{c}n 9: 9.61-17.8 \\
n 7: 0.6-0.72\end{array}$ & $40.7-44.0$ & $\begin{array}{c}n 3: 23.34-24.5 \\
n 6: 5.6-9.16\end{array}$ & $3.0-4.48$ & 0.13 & 0.71 & 0.27 & 0.09 & {$[59,63]$} \\
\hline Blueberry & $4.98-7.64$ & 0.08 & $1.93-3.31$ & $\begin{array}{c}n 9 \text { cis: } 50.74 \\
n 9 \text { trans: } 0.38 \\
n 9 \text { total: } 18.00 \\
n 7: 0.56\end{array}$ & $30.0-35.84$ & $\begin{array}{c}n 3: 7.06-36.08 \\
n 6: 0.14\end{array}$ & - & $0.19-0.49$ & 0.14 & 0.05 & 0.11 & {$[57,59]$} \\
\hline Gooseberry & 8.12 & - & 1.83 & n9: 14.32 & 33.86 & $\begin{array}{c}n 3: 20.54 \\
n 6: 8.48\end{array}$ & 5.45 & - & - & - & - & [64] \\
\hline Strawberry & 4.32 & - & 1.68 & 14.55 & 42.22 & n3: 36.48 & - & 0.71 & - & - & - & [37] \\
\hline
\end{tabular}




\subsubsection{Tocopherols and Tocotrienols}

Tocopherols and tocotrienols (also commonly called tocols or, generally, vitamin E) are phenolic compounds, lipid-soluble antioxidants. Due to their oxidation-preventive ability, they protect PUFAs from oxidation and are widely present in edible, unsaturated fatty acid-rich oils [65]. However, the loss of their antioxidant efficacy and even an adverse oxidation-promoting effect of tocopherols have been observed at high temperatures [66] or in oils enriched with high levels of tocopherols, especially in highly unsaturated oils [67]. In studies describing the decomposition of tocols during storage, some differences were found: $\alpha$-tocopherol is described as the least stable, with a rapid decrease in stability index observed, and $\delta$-tocopherol as the most stable section of tocopherols [68]. In addition, there are findings confirming that higher levels of $\alpha$-tocopherol in oils lead to a decreased oxidative stability index of oils [69]. The composition of tocols fractions may differ depending on oil processing conditions and berry cultivar. [65]. Tocopherol composition in chosen berry oils is presented in Table 2 . The values vary in relation to the species of the berries. However, certain common characteristics exist. Tocopherols reach higher concentrations than tocotrienols in all of the described studies [37,38,59,60,70]. For blackberry, raspberry, redcurrant and blackcurrant oils, in almost any case, $\gamma$-tocopherol is their main tocopherol. For chokeberry oil, the major tocols fraction was $\alpha$-tocopherol [38]. From a nutritional point of view, vitamin E proper uptake is essential, however, $\alpha$-tocopherol is the most active antioxidant in the human system and the only tocol that is able to cover human vitamin E demand [71].

Table 2. Tocopherol composition of berry oils (mg/100 g oil). (TP- tocopherols; T3- tocotrienols.)

\begin{tabular}{lccccccccc}
\hline $\begin{array}{l}\text { Source of } \\
\text { Oil }\end{array}$ & $\alpha$-TP & $\boldsymbol{\beta}$-TP & $\boldsymbol{\gamma}$-TP & $\boldsymbol{\delta}$-TP & $\boldsymbol{\alpha}$-T3 & $\beta$-T3 & $\boldsymbol{\gamma}$-T3 & $\delta$-T3 & Reference \\
\hline Chokeberry & 70.6 & 28.2 & 0.2 & 0.2 & - & - & 0.8 & - & {$[38]$} \\
\hline Raspberry & $27.74-46.1$ & 0.65 & $58.19-164.0$ & $5.83-22.59$ & - & 2.71 & 7.2 & - & {$[37,59,60]$} \\
\hline Blackberry & $0.89-2.54$ & 0.18 & $42.41-131.1$ & $3.17-6.97$ & - & 0.44 & 2.0 & - & {$[37,59]$} \\
\hline Blackcurrant & $28.85-36.9$ & $0.2-0.55$ & $23.01-55.4$ & $4.09-6.9$ & $0.09-0.1$ & $0.3-0.65$ & $0.2-0.26$ & - & {$[38,59]$} \\
\hline Redcurrant & $3.04-5.75$ & $0.56-0.79$ & $33.64-156.39$ & $19.38-41.13$ & 0.10 & 0.31 & 0.13 & 0.03 & {$[59,70]$} \\
\hline Gooseberry & 5.26 & 0.20 & 60.35 & 3.32 & - & - & - & - & {$[70]$} \\
\hline Blueberry & 0.44 & - & 3.44 & - & - & - & 33.04 & 0.6 & {$[37]$} \\
\hline Strawberry & - & - & 26.03 & 2.0 & - & - & - & - & {$[37]$} \\
\hline
\end{tabular}

\subsubsection{Sterols}

Plant-derived sterols, also called phytosterols, are amphiphilic steroid alcohols. They play a role as a plant cell membrane compound. Most common are sitosterol, stigmasterol and campesterol [72]. Table 3 presents the sterol contents of analyzed berries oils. In all of them, sterols composition corresponds with common values and the main sterol occurring is $\beta$-sitosterol. Specific sterol contents may differ depending on variety, year, fruit maturity and processing conditions, e.g., temperature [73,74]. Additionally, sterols' thermal stability depends on their structure, mainly on the number and location of double bonds. In the structure of $\beta$-sitosterol there is one double bond that results in its thermosensitivity at a medium range [75]. Afinisha-Deepam et al. [76] and T. Wang [77] reported that sterols do not affect the stability of oils, so their concentration in products does not influence oxidative reactions or the length of the shelf life of oil. However, there are also findings describing the sterol fraction stigmasterol as prooxidative at temperatures around $60^{\circ} \mathrm{C}$ but antioxidative at frying or baking temperatures around $180^{\circ} \mathrm{C}$ [78]. Furthermore, sterol esters added to oils or to different fats, e.g., margarine, can decrease their oxidation stability $[79,80]$. In human nutrition, sterols are believed to be competitors of cholesterol and as a result of that they reduce cholesterol absorption from dietary sources, which leads to reductions 
in concentrations of plasma cholesterol. This property may be useful in lipids-correlated disorders prevention and treatment, e.g., hypercholesterolemia [81,82].

Table 3. Sterol composition of lipid fraction of berry pomaces (\%).

\begin{tabular}{ccccccccc}
\hline $\begin{array}{c}\text { Source of } \\
\text { Oil }\end{array}$ & Cholesterol & Campesterol & Stigmasterol & $\begin{array}{c}\beta- \\
\text { Sitosterol }\end{array}$ & $\begin{array}{c}\text { D5- } \\
\text { Avenasterol }\end{array}$ & $\begin{array}{c}\text { D7- } \\
\text { Stigmasterol }\end{array}$ & $\begin{array}{c}\text { D7.25- } \\
\text { Stigmasterol }\end{array}$ & $\begin{array}{c}\text { Reference } \\
\text { Chokeberry }\end{array}$ \\
\hline S.95 & 5.5 & 3.85 & 81.8 & 1.85 & 1.8 & 1.8 & {$[38]$} \\
\hline Blackberry & 0.33 & $5.3-7.03$ & $1.8-4.87$ & $77.77-84.7$ & $3.02-7.0$ & 1.41 & - & {$[37,59]$} \\
\hline Raspberry & 0.43 & $4.5-4.51$ & $0.84-1.2$ & $79.6-83.95$ & $5.35-7.2$ & 1.24 & - & {$[37,59]$} \\
\hline Blackcurrant & 2.5 & 1.25 & 4.9 & 86.6 & 1.3 & 0.85 & 1.4 & {$[38]$} \\
\hline Blackcurrant & 0.31 & 8.14 & 0.42 & 81.09 & 3.10 & 1.92 & - & {$[59]$} \\
\hline Redcurrant & 0.36 & 10.01 & 0.24 & 87.58 & 0.36 & - & - & {$[59]$} \\
\hline Blueberry & 0.24 & $3.4-4.63$ & $0.3-0.37$ & $66.5-82.85$ & $2.14-13.8$ & 3.97 & - & {$[37,59]$} \\
\hline Strawberry & - & 5.4 & 2.3 & 71.1 & 8.7 & - & - & {$[37]$} \\
\hline
\end{tabular}

\section{Processing and Extraction}

Bioactive compounds may be isolated from pomaces by physical techniques, such as cold pressing, or chemical techniques, i.e., extraction, and extracts as well as oils may be products of these processes. Conventional methods of extraction may, however, require extended energy intake and use of organic solvents in large quantities. To help to reduce the environmental and financial impact of extraction processes caused by both high energy and organic solvent consumption, non-conventional extraction methods have been proposed. Particular novel extraction methods may lead to the obtention of extracts or oils with improved properties. A scheme for the procedures of extraction of bioactive compounds and oils from berry fruit by-products is presented in Figure 2.

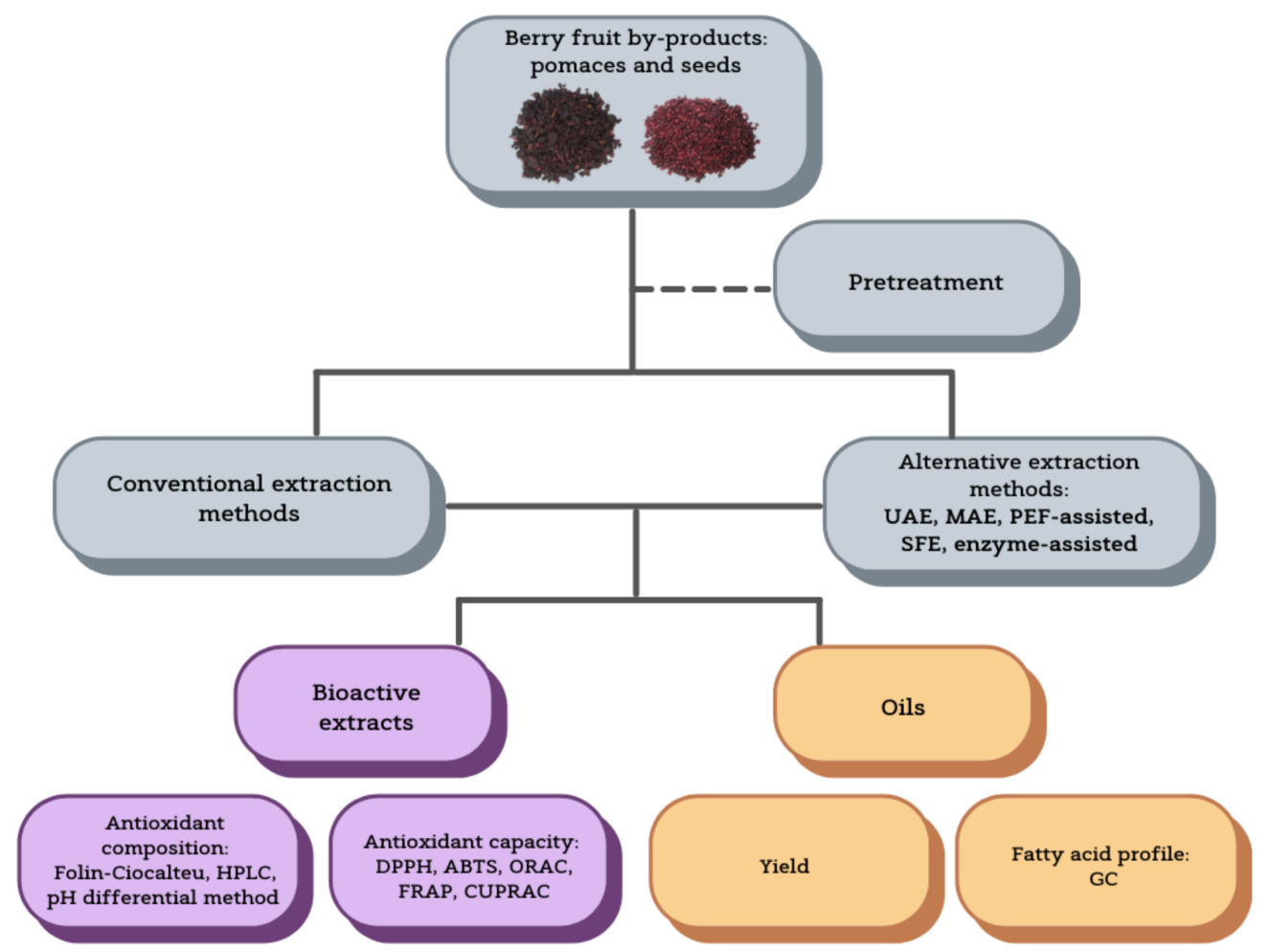

Figure 2. A scheme for the extraction procedures for bioactive compounds and oils from berry fruit by-products. 


\subsection{Conventional Extraction Methods}

Conventional, liquid-liquid and solid-liquid extraction methods are the most commonly used at a laboratory scale. In these processes, usually organic solvents, like methanol, ethanol, hexane and acetone, are used, but aqueous solvents might also be employed. The mechanism of the process involves removing a soluble fraction from an insoluble solid. The concentration of compounds released from plant tissues to the solvent reaches equilibrium with the concentrations of unreleased substances as described by the equilibrium distribution constant. Fick's second law of diffusion describes how fast the compounds are able to dissolve and reach equilibrium [83]. Factors that may improve extraction efficiency are increased solvent concentration and reduced particles of solids. Increasing solvent concentration enhances the gradient of concentrations in two phases and reducing the size of particles decreases the diffusion distance of a solution within the solid and, additionally, increases the concentration gradient, too. Temperature also helps to increase diffusivity, according to the Einstein equation. However, due to solvents' toxicity with respect to the environment or human health, conventional methods are perceived as inappropriate in the food processing industry. What is more, not all of the phenolic or lipid compounds are possible to extract using these methods. Increased temperature may also lead to damage of some thermolabile structures [83]. The efficiency of solvent extraction is determined mainly by the type of solvent and the material/solvent ratio [84]. Tables 4 and 5 compare the conditions and effectiveness (in the case of oils, yield and fatty acid profiles; in the case of phenolic extracts, antioxidant composition and antioxidant capacity of extracts) of conventional extractions of antioxidants and oils from berry by-products.

\subsubsection{Lipid Fraction}

The composition of fatty acids in extracted oils is mainly determined by the species of fruit as the source of seeds. However, the yield appears to be dependent on extraction conditions, i.e., solvent type, time of extraction and pomace pretreatment method. In the case of the solvent type, organic solvents used in conventional extraction methods are characterized by different polarities. They are defined by different dielectric constant values-a measure of solvent polarity which determines the solute-solvent correlation. The optimum dielectric constant values range from $6-8$ and result in higher oil yields. However, the higher polarity of solvents results in limitations in the solubility of extracted lipids and can lead to their hydrolysis. What is more, TAGs are amphipathic compounds and some solvents may cause hydrogen bonding of TAG ester groups. So, in conclusion, higher polarity may be a reason of lower oil yield, despite the fact that increasing the polarity of the solvent causes the opening of cell walls and improves compound release. What is more, in the case of plant oils, they also contain more polar constituents, e.g., phospholipids and tocopherols, which are the source of components that may present greater affinity to more polar solvents $[85,86]$. Hexane is a widely employed solvent in fat extraction processes due to its low polarity and it was used in most of the reviewed studies. However, it is a chemical substance with proven toxicity and water-polluting ability, so it should not be used in the food industry [87]. Considering berry seed oils extraction, ethanol is used as a solvent in the extraction of fat from blackberry seeds, resulting in higher yields compared to hexane $-11.8 \%$ and $14.2 \%$, respectively [88]. The higher oil yield value, when a more polar solvent was used, may be connected to the phenomena of partly polar compounds in the fat fraction. The time of the process is also a factor influencing efficiency. Comparing the extraction of oil from dried raspberry seeds using hexane, a higher yield was connected with a longer extraction time: the process lasting $8 \mathrm{~h}$ gave a 14.33\% yield [89] and a $2 \mathrm{~h}$ extraction resulted in a $10.7 \%$ yield [60]. The analyzed studies also showed the significant impact of seed pretreatment methods on extraction efficiency. Considering raspberry byproducts, dried pomaces [59] or seeds [60] were more susceptible to oil extraction processes than wet material [57]. In addition, replacing drying at room temperature with oven-drying resulted in higher fat yield values [89]. 


\subsubsection{Antioxidant Fraction}

Conventional extraction methods for polyphenolic compounds from berries are also conducted usually using organic solvents; however, the polarity of the solvent has to be adjusted, as polyphenols are more polar components. On a laboratory scale, alcohols are the most commonly used solvents, but these substances may still be harmful to organisms or the environment, e.g., methanol. The specific antioxidant component composition and the antioxidant capacity of obtained extracts are influenced, as in the case of oils, by the species of fruit, pretreatment methods and sample preparation. Other factors that have an influence on the properties of extracts are solvent type, solid-liquid ratio, extraction time and solvent $\mathrm{pH}$.

Table 4. Characteristics of lipid fractions extracted from berry by-products using conventional methods.

\begin{tabular}{|c|c|c|c|c|c|}
\hline Source of Waste & $\begin{array}{l}\text { Pretreatment } \\
\text { Method }\end{array}$ & $\begin{array}{c}\text { Extraction } \\
\text { Conditions } \\
\text { (S-Solvent, } \\
\text { S-L-Solid-Liquid } \\
\text { Ratio, M-Mass of } \\
\text { Solid, t-Time, } \\
\text { T-Temperature) }\end{array}$ & Oil Yield & $\begin{array}{c}\text { Fatty Acids Profile }(\%) \\
\text { (PUFA/MUFA/SFA, } \\
\text { Dominant FA) }\end{array}$ & Reference \\
\hline $\begin{array}{c}\text { Blackberry (Rubus fruticosus } \\
\text { cv. Tenac) } \\
\text { Raspberry (Rubus idaeus cv. } \\
\text { Meeker) } \\
\text { Blueberry (Vaccinium } \\
\text { myrtillus cv. Ivanhoe) } \\
\text { Blackcurrant (Ribes nigrum } \\
\text { cv. Junifer) } \\
\text { Redcurrant (Ribes rubrum cv. } \\
\text { Smoothstem) } \\
\text { pomaces }\end{array}$ & $\begin{array}{l}\text { Seeds separated from } \\
\text { pomace dried at } \\
\text { room temperature } \\
\text { using sieves, } \\
\text { then ground }\end{array}$ & $\begin{array}{c}\text { S: hexane } \\
\text { Soxhlet apparatus }\end{array}$ & $\begin{array}{c}\text { Blackberry pomace: } \\
15.68 \% \\
\text { Raspberry pomace: } \\
10.55 \% \\
\text { Blueberry pomace: } \\
13.27 \% \\
\text { Blackcurrant pomace: } \\
26.15 \% \\
\text { Redcurrant pomace: } \\
9.11 \%\end{array}$ & $\begin{array}{c}\text { Blackberry pomace } \\
\text { PUFA: } 83.78 \\
\text { MUFA: } 8.40 \\
\text { SFA: } 6.49 \\
\text { Dominant: C18:2 n6 } \\
\text { Raspberry pomace } \\
\text { PUFA: } 81.05 \\
\text { MUFA: } 12.81 \\
\text { SFA: } 4.13 \\
\text { Dominant: C18:2 n6 } \\
\text { Blueberry pomace } \\
\text { PUFA: } 72.11 \\
\text { MUFA: } 18.78 \\
\text { SFA: } 6.75 \\
\text { Dominant: C18:3 n3 } \\
\text { Blackcurrant pomace } \\
\text { PUFA: } 73.16 \\
\text { MUFA: } 14.33 \\
\text { SFA: } 6.46 \\
\text { Dominant: C18:2 n6 } \\
\text { Redcurrant pomace } \\
\text { PUFA: } 81.25 \\
\text { MUFA: } 11.13 \\
\text { SFA: } 8.39 \\
\text { Dominant } 18: 2 n 6\end{array}$ & [59] \\
\hline $\begin{array}{l}\text { Blackberry (Rubus fruticosus } \\
\text { cv. Ćačanska beztrna) } \\
\text { Raspberry (Rubus idaeus cv. } \\
\text { Willamette) seeds }\end{array}$ & $\begin{array}{l}\text { Seeds obtained from } \\
\text { the pomace dried at } \\
\text { room temperature or } \\
\text { in oven }\end{array}$ & $\begin{array}{c}\text { Standard laboratory } \\
\text { method } \\
\text { S: hexane } \\
\text { t: } 8 \mathrm{~h}\end{array}$ & $\begin{array}{c}\text { Blackberry seeds: } \\
\text { 13.97-14.34\% } \\
\text { Raspberry seeds: } \\
\text { 13.44-14.33\% } \\
\text { (Higher values for } \\
\text { pomaces dried in } \\
\text { oven) }\end{array}$ & - & [89] \\
\hline $\begin{array}{l}\text { Raspberry (Rubus idaeus) } \\
\text { different cv. seeds }\end{array}$ & $\begin{array}{l}\text { Seeds air-dried in } \\
\text { fluid bed dryer } \\
\text { for } 2 \mathrm{~h} / 25^{\circ} \mathrm{C} \text {, } \\
\text { then ground }\end{array}$ & $\begin{array}{c}\text { S: hexane } 1 \mathrm{~L} \\
\mathrm{M}: 100 \mathrm{~g} \\
\mathrm{t}: 2 \mathrm{~h} \\
\mathrm{~T}: 4^{\circ} \mathrm{C} \\
\text { Extraction performed } \\
3 \text { times }\end{array}$ & $10.7 \%$ & $\begin{array}{c}\text { Crude oil: } \\
\text { PUFA: } 83.63 \\
\text { MUFA: } 11.99 \\
\text { SFA: } 3.66 \\
\text { Dominant: } C 18: 2 n 6\end{array}$ & {$[60]$} \\
\hline
\end{tabular}


Table 4. Cont.

\begin{tabular}{|c|c|c|c|c|c|}
\hline Source of Waste & $\begin{array}{l}\text { Pretreatment } \\
\text { Method }\end{array}$ & $\begin{array}{c}\text { Extraction } \\
\text { Conditions } \\
\text { (S-Solvent, } \\
\text { S-L-Solid-Liquid } \\
\text { Ratio, M-Mass of } \\
\text { Solid, t-Time, } \\
\text { T-Temperature) }\end{array}$ & Oil Yield & $\begin{array}{c}\text { Fatty Acids Profile (\%) } \\
\text { (PUFA/MUFA/SFA, } \\
\text { Dominant FA) }\end{array}$ & Reference \\
\hline $\begin{array}{c}\text { Wild: } \\
\text { Blueberry (Vaccinium } \\
\text { myrtillus) } \\
\text { Cowberry (Vaccinium } \\
\text { vitis-idaea) } \\
\text { Raspberry (Rubus idaeus) } \\
\text { Cultivated: } \\
\text { Blueberry (Vaccinium } \\
\text { myrtillus) } \\
\text { Chokeberry (Aronia } \\
\text { melanocarpa) pomaces }\end{array}$ & $\begin{array}{l}\text { Wet pomaces } \\
\text { obtained after } \\
\text { juice pressing }\end{array}$ & $\begin{array}{l}\text { Methanol/chloroform } \\
\text { homogenization } \\
\text { procedure } \\
\text { S: methanol }(50 \mathrm{~mL}) \\
\text { and chloroform } \\
(100 \mathrm{~mL}) \\
\text { M: } 5 \mathrm{~g}\end{array}$ & $\begin{array}{c}\text { Wild } \\
\text { Blueberry pomace: } \\
3.93 \% \\
\text { Cowberry pomace: } \\
3.75 \% \\
\text { Raspberry pomace: } \\
7.00 \% \\
\text { Cultivated } \\
\text { Blueberry pomace: } \\
2.80 \% \\
\text { Chokeberry pomace: } \\
5.50 \%\end{array}$ & $\begin{array}{c}\text { Wild } \\
\text { Blueberry pomace } \\
\text { PUFA: } 41.78 \\
\text { MUFA: } 48.35 \\
\text { SFA: } 9.80 \\
\text { Dominant: C18:1 n9 } \\
\text { Cowberry pomace } \\
\text { PUFA: } 44.47 \\
\text { MUFA: } 50.87 \\
\text { SFA: } 4.65 \\
\text { Dominant: C18:1 n9 } \\
\text { Raspberry pomace } \\
\text { PUFA: } 69.00 \\
\text { MUFA: } 26.56 \\
\text { SFA: } 4.44 \\
\text { Dominant: C18:2 n6 } \\
\text { Cultivated } \\
\text { Blueberry pomace } \\
\text { PUFA: } 37.00 \\
\text { MUFA: } 51.20 \\
\text { SFA: } 11.70 \\
\text { Dominant C18:1 n9 } \\
\text { Chokeberry pomace } \\
\text { PUFA: } 65.01 \\
\text { MUFA: } 24.93 \text {, } \\
\text { SFA: } 10.06 \\
\text { Dominant: C18:2 n6 }\end{array}$ & [57] \\
\hline $\begin{array}{l}\text { Blackcurrant (Ribes nigrum } \\
\text { cv. Ben Lomond and cv. } \\
\text { Ben Tirran) pomaces }\end{array}$ & $\begin{array}{l}\text { Pomaces were } \\
\text { air-dried in oven or } \\
\text { freeze-dried and } \\
\text { (A) ground or } \\
\text { (B) seeds separated } \\
\text { from unground } \\
\text { pomace using } \\
500 \mu \mathrm{m} \text { sieve }\end{array}$ & $\begin{array}{l}\text { Soxhlet extraction } \\
\text { S: isohexane } \\
\text { M: } 10 \mathrm{~g} \\
\text { t: } 30 \mathrm{~min} \\
\text { Residue left after } \\
5 \text { cycles was } \\
\text { reground and } \\
\text { extracted in } \\
\text { another } 5 \text { cycles }\end{array}$ & $\begin{array}{l}\text { From seeds: } 14.5 \% \\
\text { from ground pomace: } \\
7.8 \%(\mathrm{w} / \text { dry })\end{array}$ & $\begin{array}{c}\text { Seeds } \\
\text { PUFA: } 79.4 \\
\text { MUFA: } 12.0 \\
\text { SFA: } 8.7 \\
\text { Dominant: C18:2 } n 6 \\
\text { Pomace } \\
\text { PUFA: } 72.0 \\
\text { MUFA: } 11.7 \\
\text { SFA: } 16.3 \\
\text { Dominant: } 18: 2 n 6\end{array}$ & [62] \\
\hline $\begin{array}{l}\text { Raspberry (Rubus idaeus) } \\
\text { dust as a by-product from } \\
\text { fruit lyophilization }\end{array}$ & $\begin{array}{l}\text { Seeds separated from } \\
\text { the dust by sieving } \\
\text { and then ground }\end{array}$ & $\begin{array}{c}\text { Soxhlet apparatus } \\
\text { S: petroleum ether } \\
\text { M: } 100 \mathrm{~g} \\
\text { t: } 6 \mathrm{~h}\end{array}$ & $14.5 \%$ & $\begin{array}{c}\text { PUFA: } 78.9 \\
\text { MUFA: } 16.9 \\
\text { SFA: } 4.2 \\
\text { Dominant: } C 18: 2 n 6\end{array}$ & [90] \\
\hline $\begin{array}{l}\text { Blackberry (Rubus fruticosus } \\
\text { cv. Cacanska beztrna) and } \\
\text { Raspberry (Rubus idaeus cv. } \\
\text { Wllamette) pomaces } \\
\text { obtained from pressing } \\
\text { long-term frozen fruits }\end{array}$ & $\begin{array}{l}\text { Pomaces dried at } \\
\text { room temperature or } \\
\text { in oven }\end{array}$ & $\begin{array}{l}\text { Standard laboratory } \\
\text { method } \\
\text { S: hexane } \\
\text { t: } 8 \mathrm{~h}\end{array}$ & - & $\begin{array}{c}\text { Blackberry pomace (room } \\
\text { temperature-dried): } \\
\text { PUFA: } 74.94 \\
\text { MUFA: } 17.87 \\
\text { SFA: } 7.13 \\
\text { Blackberry pomace } \\
\text { (oven-dried): } \\
\text { PUFA: } 75.66 \\
\text { MUFA: } 19.03 \\
\text { SFA: } 7.48 \\
\text { Dominant: C18:2 n6 } \\
\text { Raspberry pomace (room } \\
\text { temperature-dried) } \\
\text { PUFA: } 82.52 \\
\text { MUFA: } 13.21 \\
\text { SFA: } 4.23 \\
\text { Raspberry pomace } \\
\text { (oven-dried): } \\
\text { PUFA: } 87.30 \\
\text { MUFA: } 12.57 \\
\text { SFA: } 4.26 \\
\text { Dominant: } \text { C18:2 } n 6\end{array}$ & [91] \\
\hline
\end{tabular}


Table 4. Cont.

\begin{tabular}{|c|c|c|c|c|c|}
\hline Source of Waste & $\begin{array}{l}\text { Pretreatment } \\
\text { Method }\end{array}$ & $\begin{array}{c}\text { Extraction } \\
\text { Conditions } \\
\text { (S-Solvent, } \\
\text { S-L-Solid-Liquid } \\
\text { Ratio, M-Mass of } \\
\text { Solid, t-Time, } \\
\text { T-Temperature) }\end{array}$ & Oil Yield & $\begin{array}{c}\text { Fatty Acids Profile (\%) } \\
\text { (PUFA/MUFA/SFA, } \\
\text { Dominant FA) }\end{array}$ & Reference \\
\hline $\begin{array}{l}\text { Blackberry (Rubus } \\
\text { fruticosus) pomace }\end{array}$ & $\begin{array}{l}\text { Pomace dried in the } \\
\text { sun and milled }\end{array}$ & $\begin{array}{l}\text { Soxhlet apparatus } \\
\text { S: hexane } \\
250 \mathrm{~mL} / \text { ethanol } \\
250 \mathrm{~mL} \\
\text { t: } 8 \mathrm{~h}\end{array}$ & $\begin{array}{l}\text { Hexane } 11.8 \% \\
\text { Ethanol } 14.2 \%\end{array}$ & $\begin{array}{l}\text { Hexane extracted } \\
\text { PUFA: } 71.4 \\
\text { MUFA: } 17.5 \\
\text { SFA: } 11.1 \\
\text { Dominant: } 18: 2 \text { n6 } \\
\text { Ethanol extracted } \\
\text { PUFA: } 69.4 \\
\text { MUFA: } 17.4 \\
\text { SFA: } 13.2 \\
\text { Dominant: } 18: 2 n 6\end{array}$ & [88] \\
\hline $\begin{array}{c}\text { Chokeberry } \\
\text { (Aronia melanocarpa), } \\
\text { strawberry (Fragaria vesca), } \\
\text { blackcurrant (Ribes } \\
\text { nigrum) pomaces }\end{array}$ & - & $\begin{array}{c}\text { Sample homogenized } \\
\text { S: chloroform and } \\
\text { methanol }(v / v, 2: 1) \\
\text { M: } 5.0 \mathrm{~g}\end{array}$ & - & $\begin{array}{l}\text { Chokeberry pomace } \\
\text { PUFA: } 73.58 \\
\text { MUFA: } 16.91 \\
\text { SFA: } 9.51 \\
\text { Dominant: C18:2 n6 } \\
\text { Strawberry pomace } \\
\text { PUFA: } 55.77 \\
\text { MUFA: } 18.16 \\
\text { SFA: } 26.07 \\
\text { Dominant: C18:2 n6 } \\
\text { Blackcurrant pomace } \\
\text { PUFA: } 69.11 \\
\text { MUFA: } 11.56 \\
\text { SFA: } 19.33 \\
\text { Dominant: C18:2 n6 }\end{array}$ & [92] \\
\hline
\end{tabular}

Table 5. Antioxidant composition and capacity of berry by-product extracts obtained with the use of conventional methods.

\begin{tabular}{|c|c|c|c|c|c|}
\hline Source of Waste & $\begin{array}{l}\text { Pretreatment } \\
\text { Method }\end{array}$ & $\begin{array}{c}\text { Extraction } \\
\text { Conditions } \\
\text { (S-Solvent, } \\
\text { S-L-Solid-Liquid } \\
\text { Ratio, M-Mass of } \\
\text { solid, t-Time, } \\
\text { T-Temperature) }\end{array}$ & Antioxidant Composition * & Antioxidant Capacity * & Reference \\
\hline $\begin{array}{c}\text { Blackcurrant (Ribes } \\
\text { nigrum cv. Mortti), } \\
\text { Green currant (Ribes } \\
\text { nigrum cv. Verti), } \\
\text { Redcurrant (Ribes } \\
\text { rubrum cv. Red } \\
\text { Dutch), White } \\
\text { currant (Ribes rubrum } \\
\text { cv. White Dutch) } \\
\text { pomaces }\end{array}$ & $\begin{array}{l}\text { None (thawed } \\
\text { pomace) }\end{array}$ & $\begin{array}{l}\text { S: } 92 \% \text { ethanol } \\
\text { S-L: 1:2 }(w / v)\end{array}$ & $\begin{array}{c}\text { Blackcurrant pomace } \\
\text { TPC: } 55.3 \mu \text { mol GAE/g (fw) } \\
\text { White currant pomace } \\
\text { TPC: } 24.7 \mu \text { mol GAE/g } \\
\text { Redcurrant pomace } \\
\text { TPC: } 20.5 \mu \mathrm{mol} \mathrm{GAE/g} \\
\text { Green currant pomace } \\
\text { TPC: } 17.1 \mu \mu \mathrm{mol} \mathrm{GAE/g}\end{array}$ & $\begin{array}{c}\text { Blackcurrant pomace } \\
\text { TRAP: } 25,7 \mu \mathrm{mol} \mathrm{TE} / \mathrm{g}(\mathrm{fw}) \\
\text { ORAC: } 88.8 \mu \mathrm{mol} \mathrm{TE} / \mathrm{g} \\
\text { Redcurrant pomace } \\
\text { TRAP: } 11.6 \mu \mathrm{mol} \mathrm{TE} / \mathrm{g} \\
\text { ORAC: } 23.0 \mu \mathrm{mol} \mathrm{TE} / \mathrm{g} \\
\text { Green currant pomace } \\
\text { TRAP: } 8.7 \mu \mathrm{mol} \mathrm{TE} / \mathrm{g} \\
\text { ORAC: } 32.9 \mu \mathrm{mol} \mathrm{TE} / \mathrm{g} \\
\text { White currant pomace } \\
\text { TRAP: } 8.4 \mu \mathrm{mol} \mathrm{TE} / \mathrm{g} \\
\text { ORAC: } 16.8 \mu \mathrm{mol} \mathrm{TE} / \mathrm{g}\end{array}$ & [93] \\
\hline
\end{tabular}


Table 5. Cont.

\begin{tabular}{|c|c|c|c|c|c|}
\hline Source of Waste & $\begin{array}{l}\text { Pretreatment } \\
\text { Method }\end{array}$ & $\begin{array}{c}\text { Extraction } \\
\text { Conditions } \\
\text { (S-Solvent, } \\
\text { S-L-Solid-Liquid } \\
\text { Ratio, M-Mass of } \\
\text { solid, t-Time, } \\
\text { T-Temperature) }\end{array}$ & Antioxidant Composition * & Antioxidant Capacity * & Reference \\
\hline $\begin{array}{l}\text { Cranberry (Vaccinium } \\
\text { macrocarpon), } \\
\text { Blueberry (Vaccinium } \\
\text { angustifolium) } \\
\text { pomace }\end{array}$ & $\begin{array}{l}\text { Pomace } \\
\text { freeze-dried, } \\
\text { then ground }\end{array}$ & $\begin{array}{l}\text { S: } 80 \% \text { ethanol } \\
\text { S-L: } 1: 5(w / v) \\
\text { t: } 1 \mathrm{~h} \\
\text { Mixed, obtained } \\
\text { extracts were } \\
\text { freeze-dried }\end{array}$ & $\begin{array}{c}\text { Cranberry pomace } \\
\text { TPC (Folin-Ciocalteu): } 54.35 \\
\text { mg GAE/g (dm) } \\
\text { TPC (Glories): } 36.25 \mathrm{mg} \\
\text { GAE/g } \\
\text { Tartaric esters: } 10.29 \mathrm{mg} \\
\text { CAE/g } \\
\text { Flavanols: } 11.74 \mathrm{mg} \mathrm{QE} / \mathrm{g} \\
\text { TAC: } 11.14 \mathrm{mg} \mathrm{C} \mathrm{GE} / \mathrm{g} \\
\text { Tannins: } 48.09 \mathrm{mg} \text { GAE/g } \\
\text { Blueberry pomace } \\
\text { TPC (Folin-Ciocalteu): } 72.01 \\
\text { mg GAE/g } \\
\text { TPC (Glories): } 55.67 \mathrm{mg} \\
\text { GAE/g } \\
\text { Tartaric esters: } 15.03 \mathrm{mg} \\
\text { CAE/g } \\
\text { Flavanols: } 18.34 \mathrm{mg} \mathrm{QE} / \mathrm{g} \\
\text { TAC: } 38.53 \mathrm{mg} \text { C3GE/g } \\
\text { Tannins: } 58.87 \mathrm{mg} \mathrm{GAE} / \mathrm{g}\end{array}$ & $\begin{array}{c}\text { Cranberry pomace } \\
\text { ABTS: } 306.77 \mu \mathrm{mol} \mathrm{TE} / \mathrm{g}(\mathrm{dm}) \\
\text { FRAP: } 243.61 \mu \mathrm{mol} \mathrm{TE} / \mathrm{g} \\
\text { Blueberry pomace } \\
\text { ABTS: } 468.79 \mu \mathrm{mol} \mathrm{TE} / \mathrm{g} \\
\text { FRAP: } 372.22 \mu \mathrm{mol} \mathrm{TE} / \mathrm{g}\end{array}$ & [94] \\
\hline $\begin{array}{l}\text { Bilberry (Vaccinium } \\
\text { myrtillus), Blackberry } \\
\text { (Rubus fruticosus), } \\
\text { Raspberry (Rubus } \\
\text { idaeus), Strawberry } \\
\text { (Fragaria vesca) } \\
\text { pomaces }\end{array}$ & None & $\begin{array}{c}\text { S: } 80 \% \text { methanol with } \\
0.05 \% \text { acetic acid } \\
\text { M: } 20 \mathrm{~g} \\
3 \text { times extracted: } \\
60 \mathrm{~min}(160 \mathrm{~mL} \text { of } \mathrm{S}), \\
30 \mathrm{~min}(80 \mathrm{~mL} \text { of } \mathrm{S}) \\
\text { and } 30 \mathrm{~min}(80 \mathrm{~mL} \text { of } \\
\text { S) }\end{array}$ & $\begin{array}{c}\text { Bilberry pomace } \\
\text { TPC: } 11.16 \mathrm{mg} \text { GAE } / \mathrm{g}(\mathrm{fw}) \\
\text { TFC: } 10.47 \mathrm{mg} \mathrm{RE} / \mathrm{g} \\
\text { TAC: } 12.79 \mathrm{mg} \mathrm{C3GE} / \mathrm{g} \\
\text { Blackberry pomace } \\
\text { TPC: } 8.05 \mathrm{mg} \mathrm{GAE} / \mathrm{g} \\
\text { TFC: } 2.45 \mathrm{mg} \mathrm{RE} / \mathrm{g} \\
\text { TAC: } 1.49 \mathrm{mg} \mathrm{C3GE} / \mathrm{g} \\
\text { Raspberry pomace } \\
\text { TPC: } 6.38 \mathrm{mg} \mathrm{GAE} / \mathrm{g} \\
\text { TFC: } 5.92 \mathrm{mg} \mathrm{RE} / \mathrm{g} \\
\text { TAC: } 0.65 \mathrm{mg} \mathrm{C3GE} / \mathrm{g} \\
\text { Strawberry pomace } \\
\text { TPC: } 4.88 \mathrm{mg} \mathrm{GAE} / \mathrm{g} \\
\text { TFC: } 2.96 \mathrm{mg} \mathrm{RE} / \mathrm{g} \\
\text { TAC: } 0.19 \mathrm{mg} \mathrm{C} \mathrm{GE} / \mathrm{g}\end{array}$ & $\begin{array}{c}\text { Bilberry pomace } \\
\text { DPPH IC } 50: 0.040 \mathrm{mg} / \mathrm{mL} \\
\text { (pomace extract) } \\
\text { Blackberry pomace } \\
\text { DPPH IC } \\
\text { Raspberry pomace } 0.017 \mathrm{mg} / \mathrm{mL} \\
\text { DPPH IC } 50: 0.040 \mathrm{mg} / \mathrm{mL} \\
\text { Strawberry pomace } \\
\text { DPPH IC } 50: 0.038 \mathrm{mg} / \mathrm{ml}\end{array}$ & [95] \\
\hline $\begin{array}{l}\text { Blackberry (Rubus } \\
\text { fruticosus) wild and } \\
\text { cultivated (cv. } \\
\text { Cacanska Bestrna } \\
\text { and cv. Chester } \\
\text { Thornless) pomaces }\end{array}$ & - & $\begin{array}{c}\text { Soxhlet apparatus } \\
\text { S: } 80 \% \text { ethanol } \\
\text { t: } 6 \mathrm{~h} \\
\text { extracts dried in a } \\
\text { vacuum desiccator }\end{array}$ & $\begin{array}{c}\text { Blackberry cv. Chester } \\
\text { Thornless pomace } \\
\text { TPC: } 35.40 \mathrm{mg} \text { GAE/g } \\
\text { fresh pomace }(\mathrm{dm}) \\
\text { TFC: } 5.66 \mathrm{mg} \text { QE } / \mathrm{g} \\
\text { Flavanols: } 6.63 \mathrm{mg} Q \mathrm{Q} / \mathrm{g} \\
\text { Monomeric anthocyanins: } \\
\text { 17.31 mg C3GE/g } \\
\text { Blackberry cv. Cacanska } \\
\text { Bestrna pomace } \\
\text { TPC: } 26.30 \mathrm{mg} \mathrm{GAE} / \mathrm{g} \\
\text { TFC: } 3.32 \mathrm{mg} \mathrm{QE} / \mathrm{g} \\
\text { Flavanols: } 2.55 \mathrm{mg} \text { QE } / \mathrm{g} \\
\text { Monomeric anthocyanins: } \\
\text { 8.43 mg C3GE/g } \\
\text { Blackberry wild pomace } \\
\text { TPC: } 48.28-50.16 \mathrm{mg} \mathrm{GAE} / \mathrm{g} \\
\text { TFC: } 7.45-7.73 \mathrm{mg} \mathrm{QE} / \mathrm{g} \\
\text { Flavanols: } \\
\text { 6.13-6.39 mg QE/g } \\
\text { Monomeric anthocyanins: } \\
\text { 13.05-13.40 mg C3GE/g }\end{array}$ & $\begin{array}{c}\text { Blackberry cv. Chester } \\
\text { Thornless pomace } \\
\text { DPPH IC } 50.178 \mathrm{mg} / \mathrm{mL} \\
\text { (pomace extract) } \\
\text { ABTS IC } 50 \text { : } 0.035 \mathrm{mg} / \mathrm{mL} \\
\text { Blackberry cv. Cacanska } \\
\text { Bestrna pomace } \\
\text { DPPH IC } 50 \text { : } 0.206 \mathrm{mg} / \mathrm{mL} \\
\text { ABTS IC } 50.047 \mathrm{mg} / \mathrm{mL} \\
\text { Blackberry wild pomace } \\
\text { DPPH IC } \text { IC }_{50}: \\
0.106-0.127 \mathrm{mg} / \mathrm{mL} \\
\text { ABTS IC } 50 \\
0.024-0.027 \mathrm{mg} / \mathrm{mL}\end{array}$ & [28] \\
\hline $\begin{array}{c}\text { Raspberry (Rubus } \\
\text { idaeus cv. Meeker and } \\
\text { cv. Willamette) } \\
\text { pomace }\end{array}$ & None & $\begin{array}{c}\text { S: } 80 \% \text { methanol with } \\
0.05 \% \text { acetic acid } \\
\text { M: } 20 \mathrm{~g} \\
\text { T: room temperature } \\
\text { Two extractions in: } \\
160 \mathrm{~mL}, 60 \mathrm{~min} \text { and } \\
80 \mathrm{~mL}, 30 \mathrm{~min}\end{array}$ & $\begin{array}{c}\text { cv. Meeker pomace } \\
\text { TPC (HPLC): } \\
338.80 \mathrm{mg} / 100 \mathrm{~g} \text { (pomace) } \\
\text { cv. Willamette pomace } \\
\text { TPC: } 410.66 \mathrm{mg} / 100 \mathrm{~g}\end{array}$ & $\begin{array}{c}\text { cv. Meeker pomace } \\
\text { DPPH IC } \text { IC }_{50}: 0.67 \mathrm{mg} / \mathrm{mL} \\
\text { (pomace extract) } \\
\text { cv. Willamette pomace } \\
\text { DPPH IC } \text { IC }_{50}: 0.54 \mathrm{mg} / \mathrm{mL}\end{array}$ & [96] \\
\hline
\end{tabular}


Table 5. Cont.

\begin{tabular}{|c|c|c|c|c|c|}
\hline Source of Waste & $\begin{array}{l}\text { Pretreatment } \\
\text { Method }\end{array}$ & $\begin{array}{c}\text { Extraction } \\
\text { Conditions } \\
\text { (S-Solvent, } \\
\text { S-L-Solid-Liquid } \\
\text { Ratio, M-Mass of } \\
\text { solid, t-Time, } \\
\text { T-Temperature) }\end{array}$ & Antioxidant Composition * & Antioxidant Capacity * & Reference \\
\hline $\begin{array}{l}\text { Cranberry (Vaccinium } \\
\text { macrocarpon), } \\
\text { blueberry (Vaccinium } \\
\text { myrtillus) and } \\
\text { raspberry (Rubus } \\
\text { idaeus) pomace }\end{array}$ & $\begin{array}{l}\text { Dehydrated, } \\
\text { ground, } \\
\text { separated into } \\
2 \text { groups } \\
\text { depending on } \\
\text { particle size } \\
\text { (smaller } \\
\text { particle size, } \\
0.15 \mathrm{~mm}, \text { and } \\
\text { larger, } 1 \mathrm{~mm} \text { ) }\end{array}$ & $\begin{array}{c}\text { S: methanol } \\
\text { S-L: } 1: 20 \\
\text { t: } 1-24 \mathrm{~h} \\
\text { T: } 40^{\circ} \mathrm{C} \\
\text { using orbital shaker }\end{array}$ & $\begin{array}{c}\text { Obtained after the most } \\
\text { effective extraction } \\
\text { parameters } \\
\text { Cranberry pomace ( } 1 \mathrm{~h} \text {, larger } \\
\text { particles) } \\
\text { TPC: } 138 \text { mg GAE /g (fresh } \\
\text { extract) } \\
\text { Blueberry pomace }(6 \mathrm{~h} \text {, smaller } \\
\text { particles) } \\
\text { TPC: } 172 \mathrm{mg} \text { GAE } / \mathrm{g} \\
\text { Raspberry pomace }(18 \mathrm{~h}, \\
\text { Smaller particles) } \\
\text { TPC: } 270 \text { mg GAE/g }\end{array}$ & $\begin{array}{c}\text { Obtained after the most } \\
\text { effective extraction parameters } \\
\text { Cranberry pomace }(1 \mathrm{~h} \text {, larger } \\
\text { particles) } \\
\text { DPPH EC } 50: 3.73 \mathrm{mg} / \mathrm{mL} \\
\text { (pomace extract) } \\
\text { Blueberry pomace }(6 \mathrm{~h} \text {, smaller } \\
\text { particles) } \\
\text { DPPH EC } \text { ED }_{50} \text { - } \\
\text { Raspberry pomace }(18 \mathrm{~h} \text {, smaller } \\
\text { particles) } \\
\text { DPPH EC } \\
\text { 50: } 0.30 \mathrm{mg} / \mathrm{mL}\end{array}$ & [97] \\
\hline $\begin{array}{l}\text { Strawberry (Fragaria } \\
\text { vesca) pomace }\end{array}$ & $\begin{array}{c}\text { Pomace dried } \\
\text { in convection } \\
\text { dryer (temp. } \\
65-70 \mathrm{C}, 8 \mathrm{~h} \text { ), } \\
\text { sieved, } \\
\text { particles over } 5 \\
\text { mm were } \\
\text { ground to } \\
\text { obtain final } \\
\text { material, with } \\
\text { particle size } \\
\text { between } 2 \text { and } \\
5 \mathrm{~mm}\end{array}$ & $\begin{array}{c}\text { Water extraction } \\
\text { S: water } \\
\text { S-L: } 4: 1 \\
\text { M: } 1500 \mathrm{~g} \\
\text { t: } 1 \mathrm{~h} \\
\text { T: } 65-70^{\circ} \mathrm{C} \\
3 \text { times extracted } \\
\text { Ethanol extraction } \\
\text { S: } 60 \% \text { ethanol (4) } \\
\text { S-L: } 4: 1 \\
\text { M: } 3.5 \mathrm{~kg} \text { of aqueous } \\
\text { extract } \\
\text { t: } 24 \mathrm{~h} \\
\text { T: } 20^{\circ} \mathrm{C} \\
\text { Repeated once using } \\
3.5 \mathrm{~L} \text { of ethanol }\end{array}$ & $\begin{array}{c}\text { Water extract } \\
\text { TPC (HPLC): } 5.8 \mathrm{~g} / 100 \mathrm{~g} \\
\text { (dm) } \\
\text { Ethanolic extract } \\
\text { TPC: } 29.71 \mathrm{~g} / 100 \mathrm{~g}\end{array}$ & - & [98] \\
\hline $\begin{array}{c}\text { Blueberry (Vaccinium } \\
\text { myrtillus) } \\
\text { Raspberry (Rubus } \\
\text { idaeus) } \\
\text { Redcurrant (Ribes } \\
\text { rubrum) and } \\
\text { Blackberry (Rubus } \\
\text { fruticosus) pomaces }\end{array}$ & $\begin{array}{l}\text { Lyophilized } \\
\text { and ground } \\
\text { pomaces }\end{array}$ & $\begin{array}{c}\text { S: } 75 \% \text { methanol with } \\
1 \% \mathrm{HCl}(5 \mathrm{~mL}) \\
\mathrm{M}: 1 \mathrm{~g} \\
\text { t: } 12 \mathrm{~h} \\
\text { T: } 25^{\circ} \mathrm{C}\end{array}$ & $\begin{array}{c}\text { Blueberry pomace } \\
\text { TPC: } 19.55 \mathrm{mg} \text { GAE } / \mathrm{g}(\mathrm{dm}) \\
\text { TAC (HPLC): } 11.88 \mathrm{mg} / \mathrm{g} \\
\text { Raspberry pomace } \\
\text { TPC: } 20.15 \mathrm{mg} \text { GAE } / \mathrm{g} \\
\text { TAC: } 1.88 \mathrm{mg} / \mathrm{g} \\
\text { Redcurrant pomace } \\
\text { TPC: } 34.47 \mathrm{mg} \mathrm{GAE} / \mathrm{g} \\
\text { TAC: } 1.50 \mathrm{mg} / \mathrm{g} \\
\text { Blackberry pomace } \\
\text { TPC: } 17.00 \mathrm{mg} \text { GAE } / \mathrm{g} \\
\text { TAC: } 1.92 \mathrm{mg} / \mathrm{g}\end{array}$ & $\begin{array}{c}\text { Blueberry pomace } \\
\text { ABTS: } 269.8 \mu \mathrm{mol} \mathrm{TE} / \mathrm{g}(\mathrm{dm}) \\
\text { Raspberry pomace } \\
\text { ABTS: } 297.5 \mu \mathrm{mol} \mathrm{TE} / \mathrm{g} \\
\text { Redcurrant pomace } \\
\text { ABTS: } 608.3 \mu \mathrm{mol} \mathrm{TE} / \mathrm{g} \\
\text { Blackberry pomace } \\
\text { ABTS: } 225.4 \mu \mathrm{mol} \mathrm{TE} / \mathrm{g}\end{array}$ & [27] \\
\hline $\begin{array}{l}\text { Raspberry (Rubus } \\
\text { idaeus cv. Meeker and } \\
\text { cv. Willamette) }\end{array}$ & None & $\begin{array}{c}\text { S: } 80 \% \text { methanol with } \\
0.05 \% \text { acetic acid } \\
\text { M: } 70 \mathrm{~g} \\
\text { T: room temperature } \\
2 \text { times extracted } \\
\text { (A) t: } 60 \text { min, } 560 \mathrm{~mL} \\
\text { of solvent } \\
\text { (B) t: } 30 \text { min, } 280 \mathrm{~mL} \\
\text { of solvent }\end{array}$ & $\begin{array}{c}\text { cv. Meeker pomace } \\
\text { TPC: } 26.3 \mathrm{mg} \text { GAE } / \mathrm{g}(\mathrm{dm}) \\
\text { TFC: } 25.2 \mathrm{mg} \mathrm{RE} / \mathrm{g} \\
\text { TAC: } 4.28 \mathrm{mg} \mathrm{C} 3 \mathrm{GE} / \mathrm{g} \\
\text { cv. Willamette pomace } \\
\text { TPC: } 43.7 \mathrm{mg} \mathrm{GAE} / \mathrm{g} \\
\text { TFC: } 22.0 \mathrm{mg} \mathrm{RE} / \mathrm{g} \\
\text { TAC: } 2.32 \mathrm{mg} \mathrm{C} 3 \mathrm{GE} / \mathrm{g}\end{array}$ & $\begin{array}{c}\text { cv. Meeker pomace } \\
\text { DPPH EC } 50: 0.072 \mathrm{mg} / \mathrm{mL} \\
\text { (pomace extract) } \\
\text { cv. Willamette pomace } \\
\text { DPPH EC } \text { EC }_{50}: 0.042 \mathrm{mg} / \mathrm{mL}\end{array}$ & [31] \\
\hline $\begin{array}{l}\text { Blackberry (Rubus } \\
\text { fruticosus) residues } \\
\text { after pulp processing } \\
\text { and blueberry } \\
\text { (Vaccinium myrtillus) } \\
\text { residues after juice } \\
\text { processing }\end{array}$ & None & $\begin{array}{c}\text { S: ethanol } 200 \mathrm{~mL} \\
\text { M: } 5 \mathrm{~g} \\
\text { t: } 5 \mathrm{~h} \\
\text { T: } 80^{\circ} \mathrm{C} \\
\text { Soxhlet apparatus }\end{array}$ & $\begin{array}{c}\text { Blackberry pomace } \\
\text { TPC: } 7.84 \mathrm{mg} \text { GAE } / \mathrm{g}(\mathrm{dm}) \\
\text { Monomeric anthocyanins: } \\
2.82 \mathrm{mg} \text { C } 3 \mathrm{GE} / \mathrm{g} \\
\text { Blueberry pomace } \\
\text { TPC: } 6.83 \mathrm{mg} \text { GAE } / \mathrm{g} \\
\text { Monomeric anthocyanins: } \\
2.58 \mathrm{mg} \text { C } 3 \mathrm{GE} / \mathrm{g}\end{array}$ & $\begin{array}{c}\text { Blackberry pomace } \\
\text { DPPH: } 66.92 \mu \mathrm{mol} \mathrm{TE} / \mathrm{g}(\mathrm{dm}) \\
\text { ABTS: } 124.14 \mu \mathrm{mol} \mathrm{TE} / \mathrm{g} \\
\text { FRAP: } 120.90 \mu \mathrm{mol} \mathrm{TE} / \mathrm{g} \\
\text { Blueberry pomace } \\
\text { DPPH: } 40.38 \mu \mathrm{mol} \mathrm{TE} / \mathrm{g} \\
\text { ABTS: } 100.66 \mu \mathrm{mol} \mathrm{TE} / \mathrm{g} \\
\text { FRAP: } 63.90 \mu \mathrm{mol} \mathrm{TE} / \mathrm{g}\end{array}$ & [99] \\
\hline
\end{tabular}

${ }^{*}$ Results are expressed as written in the bracket after first given result. 


\subsection{Ultrasound Assisted Extraction}

Ultrasound-assisted extraction (UAE) involves using ultrasound generating devices with the proper solvent to extract bioactive compounds. In a review by Medina-Torres et al. [100], the mechanism and effects on plant tissue of UAE is summed up. UAE utilizes the phenomenon of acoustic cavitation which results in damage to the cell walls of plant material, as shown in Figure 3. This leads to increased release of bioactive compounds. The operating principles of ultrasound are mechanical waves characterized by length, amplitude, frequency, speed, power and intensity. Ultrasonic wave frequency ranges from $20 \mathrm{kHz}$ to $10 \mathrm{MHz}$. The sustainability of UAE is due to decreased solvent and energy consumption according to lower time and temperature requirements as compared to conventional extraction methods.

Table 6 presents a summary of pretreatment and extraction parameters used in ultrasound assisted extraction. UAE was successfully used as a method for the isolation of bioactive compounds from fresh berry fruits. It led to the improved yield of polyphenols (and therefore antioxidant activity) of extract obtained from chokeberry fruits. In addition, temperature increase and addition of ethanol to the solvent enhanced the efficiency of the process [101]. UAE prior to the separation and analysis of polyphenol compounds in strawberry fruits results in decreased extraction time [102]. It was also found that UAE allowed the use of a lower temperature and lower solvent concentrations in anthocyanin extraction from blueberry fruits and that it results in monomeric anthocyanin-rich extracts [103]. UAE has been applied to extract the lipid fractions from seeds other than berry fruit seeds. The crucial parameter for oil extraction yield from apricot kernels was temperature [104]. However, in UAE from papaya seeds, the most significant factors for oil extraction yield, AA and oil stability were time and temperature [105]. Fatty acid composition and the TAG profile of oil from papaya seed extracted by UAE does not vary significantly from oil extracted conventionally [106].

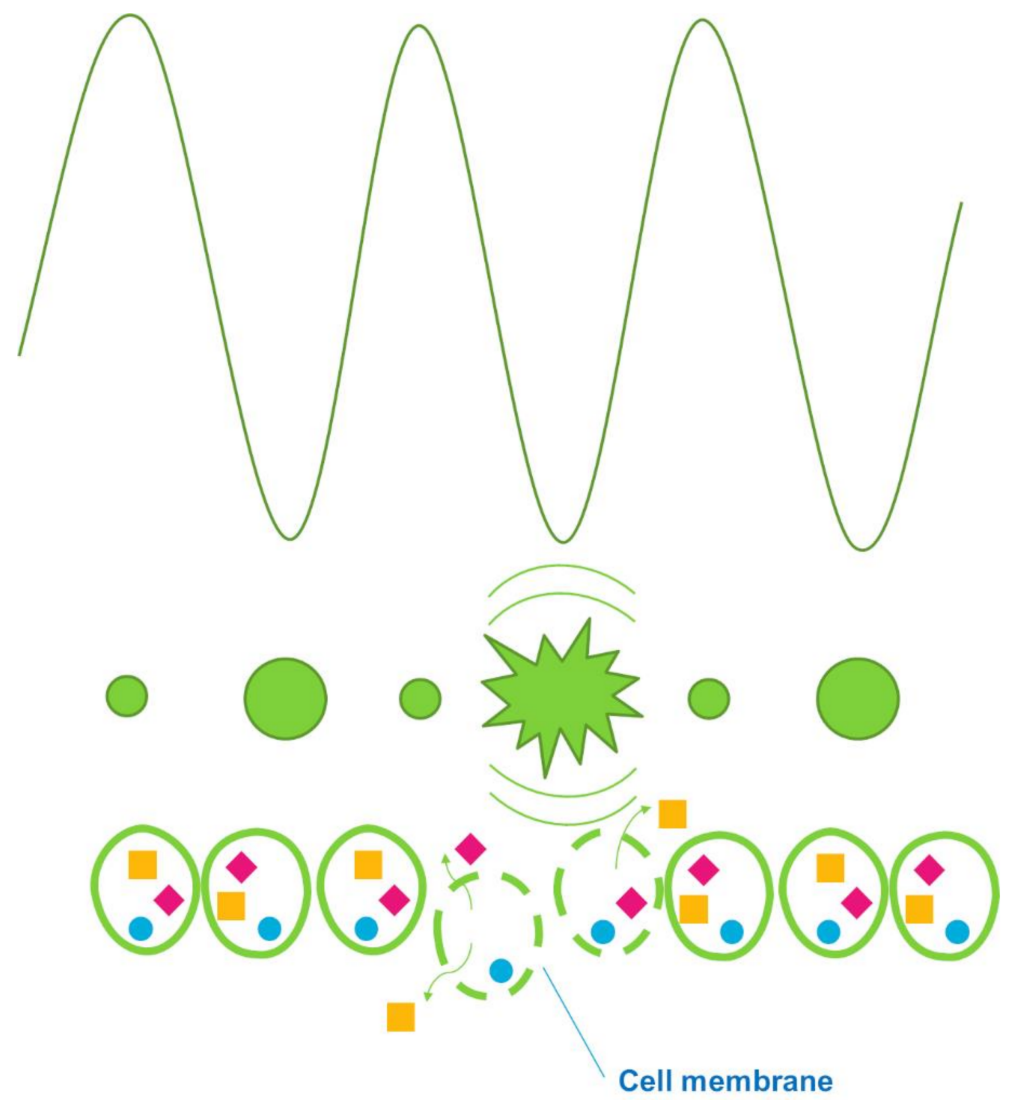

Figure 3. A scheme of ultrasound impact on the plant material. 


\subsubsection{Polyphenolic Compounds}

Krivokapić et al. [107] examined raspberry pomace extraction using the UAE method and compared it to conventional maceration. UAE was conducted in an ultrasonic cleaner for $20 \mathrm{~min}$ at $50{ }^{\circ} \mathrm{C}$ and $50 \mathrm{kHz}$ with acidulated methanol as a solvent. The obtained results showed that measured TPC, TFC and TAC were all significantly increased when UAE was used. It led to levels of $27.79 \mathrm{mg} \mathrm{GAE} / \mathrm{L}$ of extract, $8.02 \mathrm{mg} \mathrm{QE} / \mathrm{g}$ of pomace and $7.13 \mathrm{mg}$ CGE/L of extract for TPC, flavonoid content and anthocyanin content, respectively. In addition, AA measured in FRAP and DPPH assays was higher in extracts treated with ultrasound. The values obtained for FRAP and DPPH were $1002.72 \mu \mathrm{mol} \mathrm{FE} / \mathrm{L}$ of extract and $567.00 \mu \mathrm{mol} \mathrm{TE} / 100 \mathrm{~g}$ of pomace, respectively. Ellagic acid was detected by HPLC as the most abundant polyphenol. Bamba et al. [108] conducted UAE of polyphenols from blueberry wine pomace. Before extraction, the pomace was stored as freeze-dried material. In this paper, it was summarized how individual parameters of UAE—sonication time, solid-liquid ratio, solvent type, temperature and $\mathrm{pH}$-affected the quality of extracts. Qualitative parameters consisted of TPC, TFC, TAC and AA. The time of extraction significantly affected only TAC, which values were significantly higher in the case of a $90 \mathrm{~min}$ run. Solid-liquid ratio was a factor determining changes in all measured parameters. With water used as a solvent, TPC, TFC and TAC were highest for the lowest value of the solid-liquid ratio-1/40. A solid-liquid ratio of $50 \%$ ethanol at the lowest level of $1 / 20$ resulted in the highest AA, TPC and TAC in extracts. However, TFC did not follow that trend and the highest values were reached with a 1/15 ratio. In addition, concentrations for all of the compounds, as well as the AA value, were significantly higher when $50 \%$ ethanol was used in UAE. Ethanol concentration was also a parameter affecting bioactive compound yield and AA of extracts. The most effective concentration was $50 \%(v / v)$ ethanol in water. The lowest TPC, TFC, TAE and AA values were obtained using the highest concentration of ethanol-90\% $(v / v)$. pH determined significantly TPC, TAC and AA, and for TPC and AA, $\mathrm{pH} 8.3$ was the most effective; however, it resulted in decreased TAC, which was highest with $\mathrm{pH}$ 3.3. The temperature set at $60^{\circ} \mathrm{C}$ resulted in significantly increased TFC and AA but decreased TPC, for which a temperature set at $20^{\circ} \mathrm{C}$ was the most preferable. TAC was not significantly affected by temperature. The anthocyanidin profile was specified by HPLC. In both water and ethanolic extracts, the most abundant compound was malvidin and subsequently delphinidin, petunidin and cyanidin. Zafra-Rojas et al. [109] described UAE optimization using RSM. The experiment was held under constant conditions of frequency $20 \mathrm{kHz}$, S-L ratio $1: 24$ and a temperature of $4{ }^{\circ} \mathrm{C}$ at the beginning of the process and measured as $25^{\circ} \mathrm{C}$ at the end. Variable parameters were the amplitude of ultrasounds, in a range of $80-90 \%$, and the time of extraction, in a range of 10-15 min. Mathematical analysis revealed an amplitude and time of $91 \%$ and $15 \mathrm{~min}$, respectively, as the most beneficial parameters for TPC, TAC and AA extracts at $\mathrm{dm}$ basis. Predicted values were $1200 \mathrm{mg}$ GAE/100 g TPC, $380 \mathrm{mg} / 100 \mathrm{~g}$ TAC and $6300 \mu \mathrm{mol} \mathrm{TE} / 100 \mathrm{~g}$ AA in an ABTS assay and $9600 \mu \mathrm{mol} \mathrm{TE} / 100 \mathrm{~g}$ in a DPPH assay. The experimental values obtained at optimum UAE conditions were: $1201.23 \mathrm{mg} \mathrm{GAE} / 100 \mathrm{~g}, 379 \mathrm{mg} / 100 \mathrm{~g}, 6318 \mu \mathrm{mol} \mathrm{TE} / 100 \mathrm{~g}$ and $9617.22 \mu \mathrm{mol} \mathrm{TE} / 100 \mathrm{~g}$ for TPC, TAC, AA (determined by ABTS) and AA (determined by $\mathrm{DPPH})$, respectively. The UAE method was compared to conventional SLE with use of both water and ethanol. UAE yielded the highest values for TPC and AA in both assays. However, TAC in the extract obtained by UAE was not significantly different from the TAC of the extract obtained by SLE with water and was significantly lower compared to the result for SLE with ethanol. The impact of ultrasound treatment on other unconventional methods of extraction was also investigated. Xue et al. [110] combined UAE with enzymatic treatment of pectinase. The mechanism of enzyme impact on the extraction process was provided in the following text. Raspberry pomace obtained from the wine industry was freeze-dried until the moisture content was $<5 \%$. After $12 \mathrm{~h}$ at $-18{ }^{\circ} \mathrm{C}$, it was milled to $0.45 \mathrm{~mm}$ as the maximum size of particles. Conditions of UAE were determined using RSM. The most efficient parameters of anthocyanin extraction were chosen as follows: temperature $43.94{ }^{\circ} \mathrm{C}$, ultrasound power $290.9 \mathrm{~W}$, time $30 \mathrm{~min}$ and pectinase dosage $0.16 \%$. 
S-L ratio and type of solvent were constant and ranged from $1 / 30$ and $60 \%(v / v)$ acidulated ethanol, respectively. To evaluate the mathematical method the following conditions were set: temperature $44^{\circ} \mathrm{C}$, power of ultrasound $290 \mathrm{~W}$, time $30 \mathrm{~min}$ and pectinase dosage $0.16 \%$. The obtained value of anthocyanin yield was $0.853 \mathrm{mg} / \mathrm{g}$ of pomace $(\mathrm{dm}) \mathrm{vs}$. the predicted $0.888 \mathrm{mg} / 100 \mathrm{~g}$. Enzymatic extraction with US treatment was compared with conventional extraction methods: SLE with hot water, SLE with acidulated ethanol and EAE (without US). US resulted in the highest extraction efficiency and TAC values in obtained extracts. AA measured in DPPH and RP (reducing power) assays reached the highest values when US was used. The AA value of the product extracted using US, as determined in an ABTS assay, was not significantly different from conventional EAE but was significantly increased compared to the AA values of extracts obtained in both SLEs. Ramić et al. [111] used UAE to extract polyphenolic compounds from chokeberry by-products from a filter-tea factory, produced from pomace remaining after juice pressing. The most efficient parameters were chosen using RSM for every quantitative factor individually (TPC, TFC, monomeric anthocyanin content and proanthocyanins content). The common optimal parameter for all the measured properties of extracts was the temperature set at $70^{\circ} \mathrm{C}$. Other values of conditions predicted as the most efficient were as follows: for TPC $-206.64 \mathrm{~W}$ and $80.1 \mathrm{~min}$; for TFC $-210.24 \mathrm{~W}$ and $75 \mathrm{~min}$; for monomeric anthocyanins content-216 $\mathrm{W}$ and $70 \mathrm{~min}$; and for proanthocyanins content- $199.44 \mathrm{~W}$ and $70 \mathrm{~min}$ for power and time, respectively. The observed values of yields for individual compounds were $15.058 \mathrm{mg} \mathrm{GAE} / \mathrm{mL}$ extract (vs. predicted $15.41 \mathrm{mg} \mathrm{GAE} / \mathrm{mL}$ ), $10.436 \mathrm{mg} \mathrm{CE} / \mathrm{mL}$ (vs. predicted $9.86 \mathrm{mg} \mathrm{CE} / \mathrm{mL}$ ), $2.09 \mathrm{mg} \mathrm{C} 3 \mathrm{GE} / \mathrm{mL}$ (vs. predicted $2.26 \mathrm{mg} \mathrm{C} 3 \mathrm{GE} / \mathrm{mL}$ ) and $19.82 \mathrm{mg} \mathrm{CE} / \mathrm{mL}$ (vs. predicted $20.67 \mathrm{mg} \mathrm{CE} / \mathrm{mL}$ ) for TPC, TFC, monomeric anthocyanins content and proanthocyanins content, respectively. He et al. [112] used UAE to extract polyphenolic compounds from blueberry wine pomace. The experiment was designed using RSM and optimal parameters were predicted as follows: temperature $61{ }^{\circ} \mathrm{C}$, S-L 1:22, time $24 \mathrm{~min}$. It resulted in extraction yields of $16.03 \mathrm{mg}$ GAE/g pomace and $4.19 \mathrm{mg}$ C3GE/g pomace for TPC and TAC, respectively. (Predicted values were $15.81 \mathrm{mg} \mathrm{GAE} / \mathrm{g}$ pomace for TPC and $4.12 \mathrm{mg}$ C3GE/g pomace for TAC.) Compared to the conventional method, the SLE method with acidulated ethanol (70\%) used as a solvent running for $35 \mathrm{~min}$ and with applied optimal conditions for UAE (temperature- $61{ }^{\circ} \mathrm{C}$; S-L ratio-1/22), UAE appeared to be significantly more efficient, despite a shorter extraction time. There were seven anthocyanins also identified, which were present in both extracts: delphinidin-3-O-glucoside, delphindin-3-O-arabinoside, petunidin-3-O-glucoside, cyanidin-3-O-arabinoside, cyanidin3-O-glucoside, malvidin-3-O-glucoside and malvidin-3-O-arabinoside. Loncarić et al. [113] designed an experiment with different extraction methods (PEF-assisted-mentioned in the following text and high voltage electrical discharge-assisted). UAE with variable conditions of time, temperature and solvent type was conducted. The results showed that TPC, AA, TAC, flavanol and flavonol contents reached the highest levels when UAE was performed at $80^{\circ} \mathrm{C}$ and lasted $15 \mathrm{~min}$. The type of used solvent did not affect the obtained values. Only phenolic acid yield was associated with methanol used as a solvent, an applied temperature of $40^{\circ} \mathrm{C}$ and an extraction time of $15 \mathrm{~min}$. Compared to PEF-assisted and HVED-assisted extraction methods, UAE resulted in the lowest yields of polyphenolic compounds and AA.

The UAE method was also used to obtain blackberry seed extracts from three cultivars: Dircksen, Thornfree and Black Satin. Extract yields were dependent on the cultivar and, in the case of Black Satin, the highest yield of 7\% was reached. Polyphenolic compounds of obtained extracts were also determined. LC/UV/MS analysis enabled the identification of 64 polyphenols in extracts: 47 ellagitannins, 10 ellagic acid derivatives, 4 gallic acid derivatives and traces of protocatechuic, chlorogenic and salicylic acid. The most abundant compound for both Thornfree and Dircksen seed extracts was free ellagic acid and the main constituent of Black Satin seed extract was lambertianin C. Additionally, three polyphenolic compounds were successfully isolated from extracts using a semipreparative HPLC method [114]. 


\subsubsection{Lipid Fraction}

Teng et al. [115] extracted oil from raspberry seeds using UAE. In the experimental design, optimal values of time and temperature were predicted in order to obtain the highest yield, vitamin E content and AA. The parameters for time and temperature were reported as $37 \mathrm{~min}$ and $54{ }^{\circ} \mathrm{C}$, respectively. These conditions resulted in a $22.78 \%$ extraction yield (23\% predicted), $15.21 \mathrm{mg} / \mathrm{g} \mathrm{dm}$ vitamin E content $(15 \mathrm{mg} / \mathrm{g} \mathrm{dm}$ predicted) and $80.94 \mu \mathrm{mol} \mathrm{TE} / \mathrm{g} \mathrm{dm}$ AA $(81.65 \mu \mathrm{mol} \mathrm{TE} / \mathrm{g} \mathrm{dm}$ predicted). Compared to conventional SLE using the Soxhlet apparatus method, all the examined determinants were increased when US was applicated. The extract obtained using US presented an improved FA profile, containing significantly less SFA than conventionally obtained extract. However, in both extracts, the dominant FA was linoleic acid, followed by $\gamma$-linolenic acid.

\subsubsection{Impact of Processing Conditions}

The efficiency of the UAE process expressed as quantity of antioxidants and antioxidative capacity depends mostly on the species of fruit. There are also conditions of extraction that modulate the effectiveness of UAE, listed below.

Time: Researchers claim that time of sonication influenced polyphenol or lipid fraction yields. Only in the case of one analyzed study, sonication time did not influence the polyphenol content in extracts (besides TAC). With increasing sonication time, yields were higher; however, after reaching specific cutoff values, which may vary among the studies and among the examined compounds, yields decreased [109-113,115].

Temperature: The impact of temperature on extract yield and composition is reported. Generally, the relationship between increasing temperature and polyphenol yield is similar to the impact of time on the yield of extraction. With increasing temperature, polyphenol content increases but beyond a specified point starts to decrease [109,112]. Optimal temperature conditions may vary when its impact on specific fractions of polyphenols is being measured. For example, extracts with high TAC and phenolic acids are obtained at lower temperatures $[110,113]$. By contrast, higher temperatures result in increased quality and yield of oil obtained by UAE [115].

Solvent type: When different solvent types were compared, the use of ethanol resulted in the highest polyphenol yields. Reports, however, differ with respect to the data provided for the specific concentrations that are most effective in UAE. Machado et al. [99] found $70 \%$ ethanol to be optimal for polyphenol extraction yields from blackberry and blueberry pomaces, in contrast to Bamba et al.'s [108] research, which found 50\% ethanol to be optimal. However, Loncarić et al. [113] reports that solvent type did not affect specific polyphenol extraction yields except for phenolic acids, whose content was highest when methanol was used.

Table 6. Conditions and results for ultrasound-assisted extraction of oils and bioactive compounds from berry by-products.

\begin{tabular}{|c|c|c|c|c|c|}
\hline Source of Waste & $\begin{array}{c}\text { Pretreatment } \\
\text { Method }\end{array}$ & $\begin{array}{c}\text { Extraction Conditions } \\
\text { (E-Equipment, P-Power, } \\
\text { T-Temperature, t-Time, } \\
\text { f-Frequency, S-Solvent, } \\
\text { S-L-Solid-Liquid Ratio, } \\
\text { A-Amplitude) }\end{array}$ & $\begin{array}{c}\text { Antioxidant } \\
\text { Composition * }\end{array}$ & $\begin{array}{c}\text { Antioxidant } \\
\text { Capacity * }\end{array}$ & Reference \\
\hline $\begin{array}{c}\text { Raspberry } \\
\text { (Rubus idaeus) } \\
\text { pomace }\end{array}$ & None & $\begin{array}{c}\text { E: ultrasonic cleaner } \\
\text { t: } 120 \mathrm{~min} \\
\text { T: } 50^{\circ} \mathrm{C} \\
\text { f: } 50 \mathrm{kHz} \\
\text { S: acidulated methanol } \\
(80 \%)\end{array}$ & $\begin{aligned} \text { TPC: } & 27.79 \mathrm{mg} \text { GAE } / \mathrm{L} \\
& \text { (extract) } \\
\text { TFC: } & 8.02 \mathrm{mg} \mathrm{QE} / \mathrm{g} \\
& \text { (pomace) } \\
\text { TAC: } & 7.13 \mathrm{mg} \mathrm{C} 3 \mathrm{GE} / \mathrm{L} \\
& \text { (extract) }\end{aligned}$ & $\begin{array}{c}\text { FRAP: } \\
\text { 1007.72 } \mu \mathrm{mol} / \mathrm{L} \text { FRAP } \\
\text { DPPH: } \\
567.00 \mu \mathrm{mol} / 100 \mathrm{~g} \text { TE } \\
\text { DPPH IC } 50 \\
20.00 \mu \mathrm{L} / \mathrm{mL}\end{array}$ & [107] \\
\hline
\end{tabular}


Table 6. Cont.

\begin{tabular}{|c|c|c|c|c|c|}
\hline Source of Waste & $\begin{array}{l}\text { Pretreatment } \\
\text { Method }\end{array}$ & $\begin{array}{c}\text { Extraction Conditions } \\
\text { (E-Equipment, P-Power, } \\
\text { T-Temperature, t-Time, } \\
\text { f-Frequency, S-Solvent, } \\
\text { S-L-Solid-Liquid Ratio, } \\
\text { A-Amplitude) }\end{array}$ & $\begin{array}{l}\text { Antioxidant } \\
\text { Composition * }\end{array}$ & $\begin{array}{l}\text { Antioxidant } \\
\text { Capacity * }\end{array}$ & Referenc \\
\hline $\begin{array}{l}\text { Blueberry } \\
\text { (Vaccinium } \\
\text { angustifolium) } \\
\text { pomace }\end{array}$ & $\begin{array}{l}\text { Freeze-dried } \\
\text { pomace }\end{array}$ & $\begin{array}{c}\text { E: ultrasonic cleaner bath } \\
\text { S-L: } 1 / 10 ; 1 / 15 ; 1 / 20 ; 1 / 40 \\
\text { S: water or ethanol }(10 / 50 \text { or } \\
90 \% v / v \text { in water }) \\
\text { t: } 30 ; 40 ; 60 ; 90 \mathrm{~min} \\
\text { T: } 20 / 40 / 60{ }^{\circ} \mathrm{C} \\
\text { pH: } 3.3 / 5.0 / 6.3 / 8.3 \\
\text { f: } 35 \mathrm{kHz} \\
\text { Study was divided into } \\
\text { parts where one of the } \\
\text { factors was modulated } \\
\text { while the others were } \\
\text { constant }\end{array}$ & \multicolumn{2}{|c|}{ 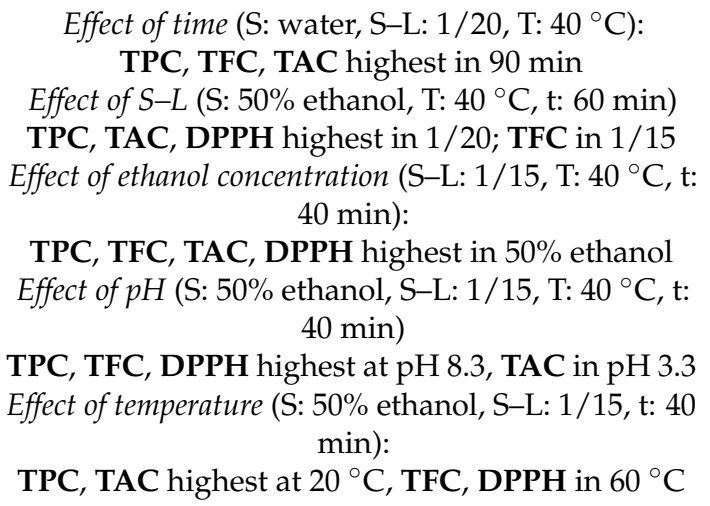 } & [108] \\
\hline $\begin{array}{c}\text { Blackberry } \\
\text { (Rubus fruticosus) } \\
\text { pomace }\end{array}$ & $\begin{array}{l}\text { Lyophilized, } \\
\text { milled and } \\
\text { sieved (500 } \\
\text { um particle } \\
\text { size) }\end{array}$ & $\begin{array}{c}\text { E: ultrasound processor } \\
\text { f: } 20 \mathrm{kHz} \\
\text { S: water } \\
\text { S-L: } 1 / 24 \mathrm{~T}: 4^{\circ} \mathrm{C} \text { (at the } \\
\text { beginning), } 25^{\circ} \mathrm{C} \text { (at the } \\
\text { end of extraction) } \\
\text { A: } 80-90 \% \\
\text { t: } 10-15 \mathrm{~min}\end{array}$ & $\begin{array}{l}\text { Optimum conditions } \\
\text { A: } 91 \% \text { and } \mathrm{t}: 15 \mathrm{~min} \\
\text { TPC: } 1201.23 \mathrm{mg} \\
\text { GAE } / 100 \mathrm{~g}(\mathrm{dm}) \\
\text { TAC: } 379.12 \mathrm{mg} / 100 \mathrm{~g}\end{array}$ & $\begin{array}{c}\text { ABTS: } 6318 \mu \mathrm{mol} \\
\text { TE/100 g }(\mathrm{dm}) \\
\text { DPPH: } 9617.22 \mu \mathrm{mol} \\
\text { TE } / 100 \mathrm{~g}\end{array}$ & [109] \\
\hline $\begin{array}{c}\text { Raspberry } \\
\text { (Rubus idaeus) } \\
\text { pomace }\end{array}$ & $\begin{array}{l}\text { Freeze-dried, } \\
\text { milled } \\
(0.45 \mathrm{~mm})\end{array}$ & $\begin{array}{c}\text { UAE combined with } \\
\text { enzymatic extraction } \\
\text { E: not specified } \\
\text { S-L: } 1 / 30 \\
\text { S: acidulated } 60 \% \\
\text { ethanol }(v / v) \\
\text { t: } 20 / 30 / 40 \mathrm{~min} \\
\text { T: } 40 / 45 / 50{ }^{\circ} \mathrm{C} \\
\text { +enzyme (pectinase dosage): } \\
0.10 / 0.15 / 0.20 \%\end{array}$ & $\begin{array}{c}\text { Optimum conditions } \\
\text { T: } 44^{\circ} \mathrm{C}, \mathrm{P}: 290 \mathrm{~W}, \\
\text { t: } 30 \mathrm{~min}, \\
\text { pectinase dosage: } \\
0.16 \% \\
\text { Anthocyanin yield: } \\
0.853 \mathrm{mg} / \mathrm{g}(\mathrm{fw})\end{array}$ & $\begin{array}{c}\text { DPPH: } 417.15 \mathrm{TE} / \mathrm{g} \\
\text { (extract) } \\
\text { ABTS: } 520.07 \mathrm{TE} / \mathrm{g}\end{array}$ & [110] \\
\hline $\begin{array}{l}\text { Chokeberry } \\
\text { (Aronia } \\
\text { melanocarpa) } \\
\text { by-products } \\
\text { from filter-tea } \\
\text { production (tea } \\
\text { produced } \\
\text { from pomace) }\end{array}$ & None & $\begin{array}{c}\text { M: } 10.0 \mathrm{~g} \\
\text { S: } 50 \% \text { ethanol } \\
\text { E: sonication water bath } \\
\text { S-L: } 1 / 5 \\
\text { f: } 40 \mathrm{kHz} \\
\text { P: } 72 / 144 / 216 \mathrm{~W} \\
\text { T: } 30 / 50 / 70^{\circ} \mathrm{C} \\
\text { t: } 30 / 60 / 90 \mathrm{~min}\end{array}$ & $\begin{array}{c}\text { Optimum conditions } \\
\text { for each property in } \\
\text { brackets } \\
\text { TPC: } \\
\text { 15.058 mg GAE } / \mathrm{mL} \\
\text { (extract) (P: } 206.64 \mathrm{~W} \text {, } \\
\left.\mathrm{T}: 70{ }^{\circ} \mathrm{C}, \mathrm{t}: 80.1 \mathrm{~min}\right) \\
\text { TFC: } \\
10.436 \mathrm{mg} \mathrm{CE} / \mathrm{mL} \\
\left(\mathrm{P}: 210.24 \mathrm{~W}, \mathrm{~T}: 70{ }^{\circ} \mathrm{C},\right. \\
\text { t: } 75 \mathrm{~min}) \\
\text { Monomeric } \\
\text { anthocyanins: } \\
2.09 \mathrm{mg} \mathrm{C} 3 \mathrm{GE} / \mathrm{mL} \\
\text { (P: } 216 \mathrm{~W}, \mathrm{~T}: 70{ }^{\circ} \mathrm{C}, \\
\text { t: } 45.6 \mathrm{~min}) \\
\text { Proanthocyanins: } \\
19.82 \mathrm{mg} \mathrm{CE} / \mathrm{mL} \\
\left(\mathrm{P}: 199.44 \mathrm{~W}, \mathrm{~T}: 70{ }^{\circ} \mathrm{C},\right. \\
\text { t: } 89.7 \mathrm{~min})\end{array}$ & - & [111] \\
\hline
\end{tabular}


Table 6. Cont.

\begin{tabular}{|c|c|c|c|c|c|}
\hline Source of Waste & $\begin{array}{l}\text { Pretreatment } \\
\text { Method }\end{array}$ & $\begin{array}{c}\text { Extraction Conditions } \\
\text { (E-Equipment, P-Power, } \\
\text { T-Temperature, t-Time, } \\
\text { f-Frequency, S-Solvent, } \\
\text { S-L-Solid-Liquid Ratio, } \\
\text { A-Amplitude) }\end{array}$ & $\begin{array}{l}\text { Antioxidant } \\
\text { Composition * }\end{array}$ & $\begin{array}{l}\text { Antioxidant } \\
\text { Capacity }{ }^{*}\end{array}$ & Reference \\
\hline $\begin{array}{c}\text { Blueberry } \\
\text { (Vaccinium ashei) } \\
\text { pomace }\end{array}$ & $\begin{array}{l}\text { Pomace dried } \\
\text { in air- } \\
\text { circulating } \\
\text { oven at } 30^{\circ} \mathrm{C} \\
\text { for } 48 \mathrm{~h} \text {, then } \\
\text { milled }\end{array}$ & $\begin{array}{c}\text { E: not specified } \\
\text { S: acidulated ethanol }(70 \% \\
v / v) \\
\text { P: } 400 \mathrm{~W} \\
\text { T: } 50 / 60 / 70{ }^{\circ} \mathrm{C} \\
\text { t: } 15 / 25 / 35 \mathrm{~min} \\
\text { S-L: } 1 / 15,1 / 20,1 / 25\end{array}$ & $\begin{array}{c}\text { Optimal conditions T: } \\
61^{\circ} \mathrm{C}, \mathrm{S}-\mathrm{L}: 1 / 22 \\
\text { TPC: } 16.03 \mathrm{mg} \text { GAE } / \mathrm{g} \\
\text { (pomace) } \\
\text { TAC: } 4.19 \mathrm{mg} \mathrm{C} 3 \mathrm{GE} / \mathrm{g}\end{array}$ & - & [112] \\
\hline $\begin{array}{l}\text { Blueberry } \\
\text { pomace }\end{array}$ & $\begin{array}{l}\text { Freeze-dried, } \\
\text { milled }\end{array}$ & $\begin{array}{c}\text { E: ultrasonic bath } \\
\text { F: } 35 \mathrm{kHz} \\
\text { S: acidulated ethanol } \\
(50 \%) / \text { acidulated methanol } \\
(50 \%) \\
\text { t: } 5 / 10 / 15 \mathrm{~min} \\
\text { T: } 20 / 40 / 80^{\circ} \mathrm{C}\end{array}$ & $\begin{array}{c}\text { Highest values for: } \\
\text { TPC: } 5.46 \mathrm{mg} \text { GAE/g } \\
(\mathrm{dm})(\text { Ethanol, } 15 \mathrm{~min} \text {, } \\
\left.80{ }^{\circ} \mathrm{C}\right) \\
\text { TAC: } 953.91 \mu \mathrm{g} / \mathrm{g} \\
\text { (Methanol, } 15 \mathrm{~min}, 80 \\
\left.{ }^{\circ} \mathrm{C}\right) \\
\text { Phenolic acids: } 561.26 \\
\mu \mathrm{g} / \mathrm{g} \text { (Methanol, } 15 \\
\left.\text { min, } 80^{\circ} \mathrm{C}\right) \\
\text { Flavanols: } 156.04 \\
\mu \mathrm{g} / \mathrm{g}(\text { Methanol, } 15 \\
\left.\text { min, } 80^{\circ} \mathrm{C}\right) \\
\text { Flavonols: } 98.63 \mu \mathrm{g} / \mathrm{g} \\
\text { (Methanol, } 15 \mathrm{~min}, 80 \\
\left.{ }^{\circ} \mathrm{C}\right)\end{array}$ & $\begin{array}{l}\text { Highest values for: } \\
\text { DPPH: } 0.25 \text { mmol TE/g } \\
\text { (dm) (Methanol, } 15 \mathrm{~min} \text {, } \\
\left.80^{\circ} \mathrm{C}\right)\end{array}$ & [113] \\
\hline $\begin{array}{c}\text { Blueberry } \\
\text { (Vaccinium } \\
\text { myrtillus) } \\
\text { pomace and } \\
\text { blackberry } \\
\text { (Rubus fruticosus) } \\
\text { residues after } \\
\text { pulp processing }\end{array}$ & None & $\begin{array}{c}\text { E: ultrasonic bath } \\
\text { F: } 37 \mathrm{kHz} \\
\text { P: } 580 \mathrm{~W} \\
\text { S-L: } 1 / 22,5 \\
\text { S: ethanol } 50 \% / \text { ethanol } \\
\text { 70\%/acidified water } \\
\text { t: } 90 \mathrm{~min} \\
\text { T: } 80^{\circ} \mathrm{C}\end{array}$ & $\begin{array}{c}\text { Blackberry residues } \\
\text { Ethanol 50\% } \\
\text { TPC: } \\
5.28 \text { mg GAE/g (dm) } \\
\text { Monomeric } \\
\text { anthocyanins: } \\
\text { 2.37 mg C3GE/g } \\
\text { Ethanol 70\% } \\
\text { TPC: } 5.86 \text { mg GAE/g } \\
\text { Monomeric } \\
\text { anthocyanins: } \\
\text { 2.38 mg C3GE/g } \\
\text { Acidified water } \\
\text { TPC: } 2.08 \text { mg GAE/g } \\
\text { Monomeric } \\
\text { anthocyanins: } \\
1.26 \text { mg C3GE/g } \\
\text { Blueberry residues } \\
\text { Ethanol } 50 \% \\
\text { TPC: } 4.40 \text { mg GAE/g } \\
\text { Monomeric } \\
\text { anthocyanins: } \\
\text { 2.07 mg C3GE/g } \\
\text { Ethanol } 70 \% \\
\text { TPC: } 5.75 \text { mg GAE/g } \\
\text { Monomeric } \\
\text { anthocyanins: } \\
2.33 \text { mg C3GE/g } \\
\text { Acidified water } \\
\text { TPC: } 2.47 \text { mg GAE/g } \\
\text { Monomeric } \\
\text { anthocyanins: } \\
1.36 \text { mg C3GE/g }\end{array}$ & 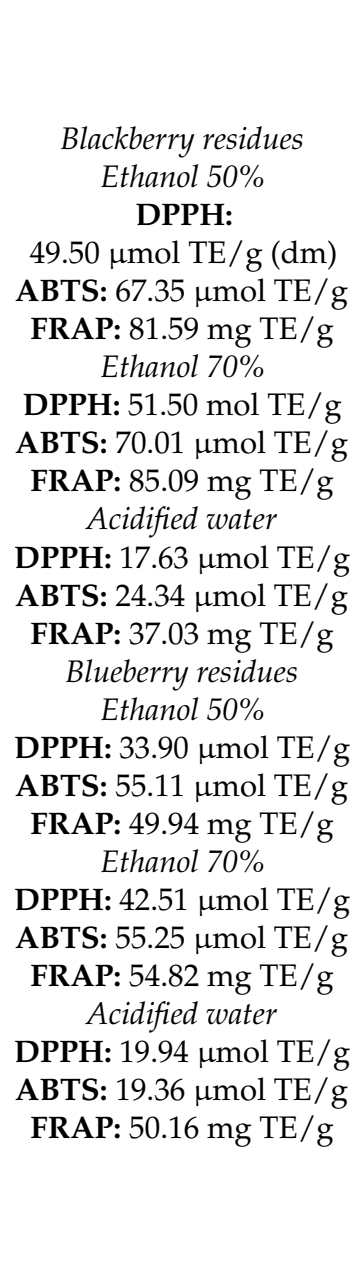 & [99] \\
\hline
\end{tabular}


Table 6. Cont.

\begin{tabular}{|c|c|c|c|c|c|}
\hline Source of Waste & $\begin{array}{l}\text { Pretreatment } \\
\text { Method }\end{array}$ & $\begin{array}{c}\text { Extraction Conditions } \\
\text { (E-Equipment, P-Power, } \\
\text { T-Temperature, t-Time, } \\
\text { f-Frequency, S-Solvent, } \\
\text { S-L-Solid-Liquid Ratio, } \\
\text { A-Amplitude) }\end{array}$ & $\begin{array}{l}\text { Antioxidant } \\
\text { Composition * }\end{array}$ & $\begin{array}{l}\text { Antioxidant } \\
\text { Capacity * }\end{array}$ & Referenc \\
\hline $\begin{array}{l}\text { Chokeberry } \\
\quad \text { (Aronia } \\
\text { melanocarpa cv. } \\
\text { Nero) pomace }\end{array}$ & $\begin{array}{l}\text { Freeze-dried } \\
\text { and ground } \\
\text { pomace }\end{array}$ & $\begin{array}{c}\text { E: ultrasonic processor } \\
\text { t: } 10 / 13 / 20 / 27 / 30 \mathrm{~min} \\
\text { S-L: } 1 / 10 \\
\text { S: } 60 / 65 / 78 / 90 / 96 \% \\
\text { ethanol } \\
\text { T: } 25^{\circ} \mathrm{C} \\
\text { A: } 50 \mu \mathrm{m}\end{array}$ & $\begin{array}{l}\text { Highest values for: } \\
\text { TPC: } 188 \mathrm{mg} \text { GAE/g } \\
(\mathrm{dm})(60 \% \text { ethanol, } 20 \\
\text { min) } \\
\text { TAC: } 89.3 \mathrm{mg} \mathrm{C3GE/g} \\
\text { (65\% ethanol, } 13 \mathrm{~min})\end{array}$ & $\begin{array}{l}\text { Highest values for } \\
\text { DPPH: } 49.2 \mathrm{mmol} \\
\text { TE/100 } \mathrm{g}(\mathrm{dm}) \\
\text { (60\% ethanol, } 20 \mathrm{~min})\end{array}$ & [116] \\
\hline $\begin{array}{l}\text { Chokeberry } \\
\text { (Aronia } \\
\text { melanocarpa) } \\
\text { pomace }\end{array}$ & - & $\begin{array}{l}\text { E: horn-type transducer } \\
\text { with cooling bath } \\
\text { S: ethanol-water (1:1)S-L: } \\
1 / 10 \\
\text { f: } 20 \mathrm{kHz} \\
\text { A: } 14 \mu \mathrm{m} \\
\text { t: } 600 \mathrm{~s}\end{array}$ & $\begin{array}{c}\text { TPC: } 1046 \mathrm{mg} / \mathrm{L} \text { GAE } \\
\text { Monomeric } \\
\text { anthocyanins: } 631 \\
\text { mg/L C3GE }\end{array}$ & - & [32] \\
\hline $\begin{array}{l}\text { Chokeberry } \\
\text { (Aronia } \\
\text { melanocarpa) } \\
\text { stems }\end{array}$ & Dried stems & $\begin{array}{l}\text { E: ultrasonic water bath } \\
\text { S: water } \\
\text { t: } 30 \mathrm{~min} \\
\text { S-L: } 1 / 25\end{array}$ & $\begin{array}{c}\text { TPC: } \\
5.22 \mathrm{mg} \mathrm{GAE} / \mathrm{g}(\mathrm{dm}) \\
\text { TFC: } 3.94 \mathrm{mg} \mathrm{RE} / \mathrm{g}\end{array}$ & $\begin{array}{c}\text { ABTS IC } \text { I0 }: \\
10.09 \mu \mathrm{g} / \mathrm{mL} \text { (extract) }\end{array}$ & [117] \\
\hline $\begin{array}{l}\text { Chokeberry } \\
\text { (Aronia } \\
\text { melanocarpa cv. } \\
\text { Galicjanka) } \\
\text { pomaces } \\
\text { obtained from } \\
\text { juice pressing } \\
\text { from crushed } \\
\text { and } \\
\text { uncrushed fruits }\end{array}$ & $\begin{array}{l}\text { Freeze-dried, } \\
\text { ground } \\
\text { pomaces }\end{array}$ & $\begin{array}{l}\text { E: ultrasonic bath } \\
\text { S: methanol with } 2 \% \\
\text { formic acid } \\
\text { S-L: } 1 / 25 \\
\text { t: } 25 \mathrm{~min}\end{array}$ & $\begin{array}{c}\text { Pomace from crushed } \\
\text { fruits } \\
\text { TPC (UPLC): } \\
15.61 \mathrm{~g} / 100 \mathrm{~g}(\mathrm{dm}) \\
\text { Pomace from uncrushed } \\
\text { fruits } \\
\text { TPC: } 24.45 \mathrm{~g} / 100 \mathrm{~g}\end{array}$ & $\begin{array}{l}\text { Pomace from crushed fruits } \\
\text { ABTS: } \\
59.94 \text { mmol TE/100 } \mathrm{g}(\mathrm{dm}) \\
\text { FRAP: } \\
32.61 \mathrm{mmol} \text { TE } / 100 \mathrm{~g} \\
\text { Pomace from uncrushed } \\
\text { fruits } \\
\text { ABTS: } \\
\text { 81.63 mmol TE/100 g } \\
\text { FRAP: } \\
52.22 \text { mmol TE/100 g }\end{array}$ & [118] \\
\hline \multicolumn{6}{|c|}{ Lipid fractions } \\
\hline Source of waste & $\begin{array}{l}\text { Pretreatment } \\
\text { method }\end{array}$ & Procedure & Oil yield & $\begin{array}{c}\text { Fatty acid composition } \\
(\%)\end{array}$ & \\
\hline $\begin{array}{c}\text { Raspberry } \\
\text { (Rubus coreanus) } \\
\text { seeds }\end{array}$ & $\begin{array}{l}\text { Seeds dried } \\
\text { in a } \\
\text { convection } \\
\text { oven at } 60^{\circ} \mathrm{C} \\
\text { for } 24 \mathrm{~h}, \\
\text { then milled }\end{array}$ & $\begin{array}{c}\text { E: sonication cleaning bath } \\
\text { f: } 40 \mathrm{kHz} \\
\text { P: } 250 \mathrm{~W} \\
\text { S-L: } 1 / 40 \\
\text { S: ethanol } \\
\text { t: } 10 / 20 / 30 / 40 / 50 \mathrm{~min} \\
\text { T: } 30 / 40 / 50 / 60 / 70^{\circ} \mathrm{C}\end{array}$ & $\begin{array}{l}\text { Optimal conditions: } \\
54{ }^{\circ} \mathrm{C}, 37 \mathrm{~min} \\
22.78 \%\end{array}$ & $\begin{array}{c}\text { SFA: } 2.45 \\
\text { MUFA: } 0.55 \\
\text { PUFA: } 92.25\end{array}$ & [115] \\
\hline
\end{tabular}

* Results are expressed as written in the bracket after first given result.

\subsection{Pulsed Electric Field-Assisted Extraction}

A review by Kumari et al. [119] described a pulsed electric field (PEF) applied in short duration pulses of moderate voltage to a material placed between two electrodes. The effect of PEF includes electroporation caused by damage to cell membranes. The formation of pores leads to mechanical breakdown of cell membranes and the material is defined as disintegrated, as presented at Figure 4. Factors involved in PEF-assisted extraction are the intensity of the electric field, the duration of treatment, the waveform of the pulse, conductivity, the porosity of the material, $\mathrm{pH}$ and the ionic strength of the solvent. PEF 
technology results in improved extraction of intracellular compounds due to increased diffusivity of intracellular substances and increased mass transfer rates. The conditions and effects of PEF applied in the pretreatment of berry fruits before juice pressing on antioxidant content and antioxidant capacity in pomace extracts are summarized in Table 7.

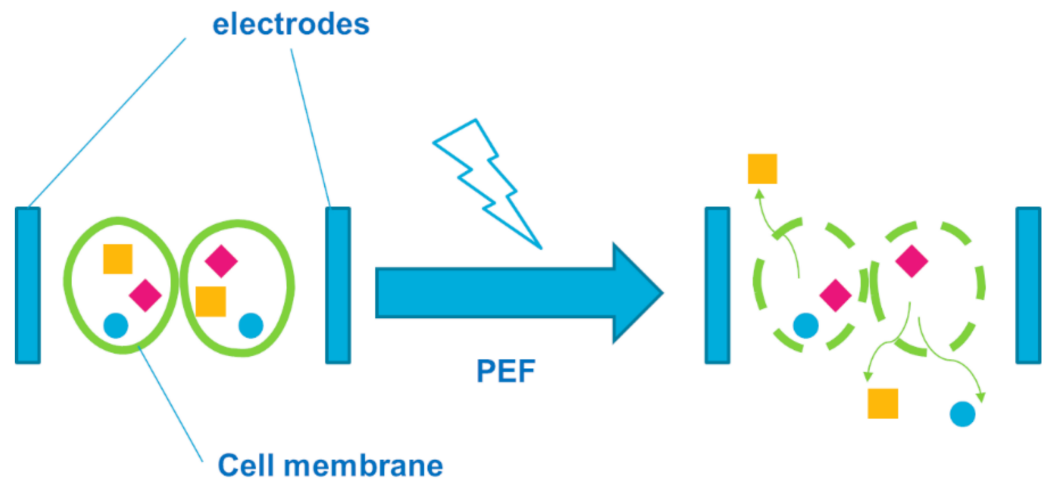

Figure 4. A scheme of PEF impact on plant material.

PEF was applied to improve the extraction of juice from blueberries. Anthocyanins from blueberry pomace were investigated by Pataro et al. [120]. Blueberries, cut in half, were pretreated by PEF with different input energy values - $1 \mathrm{~kJ} / \mathrm{kg}, 5 \mathrm{~kJ} / \mathrm{kg}$ or $10 \mathrm{~kJ} / \mathrm{kg}$ - before juice pressing. In all the samples of pomace obtained after pressing blueberries pretreated with PEF, TAC and AA were increased compared to the control (a sample of pomace remaining after pressing the juice from blueberries untreated with PEF). A correlation between increased energy input and increased values of both TAC and AA was noticed. PEF was also applied to crushed blueberries before juice-pressing in a study conducted by Bobinaitè et al. [121]. An influence of field strength value on the SLE of pomace efficiency was studied and it was found that the highest used field strength $-5 \mathrm{kV} / \mathrm{cm}$-resulted in the highest antioxidant content and capacity of blueberry pomace extracts. The values of antioxidant content and antioxidant activity were increased compared to extracts obtained from blueberry pomace untreated with PEF before juice pressing. Loncarić et al. [113] applied PEF to freeze-dried and milled pomace, which was then extracted (SLE) with acidulated ethanol or with acidulated methanol used as a solvent. The correlation between specific phenolic yields and variable numbers of pulses, energy inputs and solvent types was studied. For TPC, phenolic acids and flavonols, the most suitable conditions were: 100 pulses, a field strength of $20 \mathrm{kV} / \mathrm{cm}$ and acidulated ethanol used as a solvent. For TAC and flavanols, the number of pulses and the field strength defined as most efficient did not change, but acidulated methanol was preferable as a solvent. The DPPH of an extract was highest $(830 \mu \mathrm{mol} \mathrm{TE} / \mathrm{g} \mathrm{dm})$ when the number of pulses was 100 , the field strength was $20 \mathrm{kV} / \mathrm{cm}$ and acidulated ethanol was used as a solvent. Another example of PEF application in antioxidant extraction from blueberry pomace was described by Zhou et al. [122]. The researchers treated with PEF pomace thawed to a liquid state and ground in a colloid mill. The influence of variable conditions of treatment on anthocyanin yield was studied: the number of pulses, field strength and liquid-liquid ratio. The highest extraction yields were obtained after 10 pulses of PEF with a field strength of $20 \mathrm{kV} / \mathrm{cm}$ and using acidic ethanol in a ratio of one-to-six. Compared with UAE, PEF extraction resulted in higher anthocyanin extract yields despite a shorter time and lower temperature for the process.

As the above results for PEF-assisted extraction from by-products only concern blueberry pomace treatment, an example of extraction of polyphenolic compounds from blackcurrant juice preceded by PEF application could be mentioned (not included in the following table). The PEF procedure was conducted before juice production under optimized conditions, as determined by RSM modelling. The chosen conditions for the electric field- 
$1318 \mathrm{~V} / \mathrm{cm}$ and 315 pulses (pulse width $100 \mathrm{~ms}$ )—followed by methanol (for TPC and AA determination) or ethanol (for monomeric anthocyanin content determination) liquid extraction of blackcurrant juice resulted in extracts with significantly lowered $\mathrm{pH}$ but increased TPC, AA and monomeric anthocyanin content compared to the control (blackcurrant juice untreated with PEF) [123]. Conditions of extraction also influence the effectiveness of PEF, as listed below.

\section{Impact of Processing Conditions}

Field strength: Most studies show that a higher field strength results in increased antioxidant compound yields. When field strength is greater, the potential difference outside and inside the cell membrane is higher than the critical membrane potential and this improves the dissolution rate of the cell membrane. However, applying too strong a field may promote antioxidant degradation [119].

Number of pulses: Study results are inconclusive regarding the influence of the number of PEF pulses on the antioxidant content and capacity of extracts. Loncarić et al. [113] claim that the highest number of pulses used (100) resulted in the highest antioxidant content and capacity, while Zhou et al. [122] claim that 12 pulses was less efficient than 10, this being the optimal number of PEF pulses for the anthocyanin extraction process.

Solvent type: The properties of solvents which may determine extraction efficiency are described in the paragraph focused on SLE.

Table 7. Conditions and results of pulsed electric field-assisted extraction of bioactive compounds from berry by-products.

\begin{tabular}{|c|c|c|c|c|c|}
\hline Material & $\begin{array}{c}\text { PEF Conditions } \\
\text { (E-Equipment, P-Power, } \\
\text { FS-Field Strength, } \\
\text { f-Frequency, W-Pulse } \\
\text { Width, I-Energy Input, } \\
\text { S-Solvent, } \\
\text { L-L-Liquid-Liquid Ratio) }\end{array}$ & Procedure & $\underset{*}{\text { Antioxidant Composition }}$ & Antioxidant Activity * & Reference \\
\hline $\begin{array}{c}\text { Blueberry } \\
\text { (Vaccinium } \\
\text { myrtillus) }\end{array}$ & $\begin{array}{c}\text { E: generator of monopolar } \\
\text { square wave pulses } \\
\text { FS: } 3 \mathrm{kV} / \mathrm{cm} \\
\text { f: } 10 \mathrm{~Hz} \\
\text { W: } 20 \mu \mathrm{s} \\
\text { I: } 1 / 5 / 10 \mathrm{~kJ} / \mathrm{kg} \\
\end{array}$ & $\begin{array}{l}\text { PEF was applied to } \\
\text { blueberry fruits cut } \\
\text { in half, before } \\
\text { pressing, obtained } \\
\text { pomace examined }\end{array}$ & $\begin{array}{c}\text { Optimum conditions I: } \\
10 \mathrm{~kJ} / \mathrm{kg} \\
\text { TAC (HPLC): } \\
1574.1 \mathrm{mg} / 100 \mathrm{~g} \text { (pomace) }\end{array}$ & $\begin{array}{c}\text { Optimum conditions I: } \\
10 \mathrm{~kJ} / \mathrm{kg} \\
\text { DPPH: } 34.2 \mu \mathrm{mol} \mathrm{TE} / \mathrm{g} \\
\quad \text { (pomace) } \\
\text { FRAP: } 68.0 \mu \mathrm{mol} \mathrm{TE} / \mathrm{g}\end{array}$ & [120] \\
\hline $\begin{array}{c}\text { Blueberry } \\
\text { (Vaccinium } \\
\text { myrtillus) }\end{array}$ & $\begin{array}{c}\text { E: cylindrical PEF treatment } \\
\text { chamber (monopolar square } \\
\text { pulses) } \\
\text { FS: } 1 / 3 / 5 \mathrm{kV} / \mathrm{cm} \\
\text { f: } 10 \mathrm{~Hz} \\
\mathrm{~W}: 20 \mu \mathrm{s} \\
\text { P: } 20 \mathrm{~kW} \text { (average) } \\
\text { I: } 10 \mathrm{~kJ} / \mathrm{kg}\end{array}$ & $\begin{array}{l}\text { PEF applied to } \\
\text { crushed fresh berries } \\
\text { before juice pressing. } \\
\text { By-product was } \\
\text { extracted in SLE and } \\
\text { examined. }\end{array}$ & $\begin{array}{c}\text { Optimum conditions FS: } \\
5 \mathrm{kV} / \mathrm{cm} \\
\text { TPC: } \\
1782.64 \mathrm{mg} \mathrm{GAE} / 100 \mathrm{~g}(\mathrm{fw}) \\
\text { TAC: } 1698.55 \mathrm{mg} / 100 \mathrm{~g}\end{array}$ & $\begin{array}{c}\text { Optimum conditions FS: } \\
5 \mathrm{kV} / \mathrm{cm} \\
\text { FRAP: } \\
\text { ca. } 72 \mu \mathrm{mol} \mathrm{TE} / \mathrm{g}(\mathrm{fw})\end{array}$ & [121] \\
\hline $\begin{array}{l}\text { Blueberry } \\
\text { pomace }\end{array}$ & $\begin{array}{c}\text { E: laboratory PEF treatment } \\
\text { chamber } \\
\text { Pulse duration: } 2 \mu \mathrm{s} \\
\text { No. of pulses: } 10 / 50 / 100 \\
\text { FS: } 10 / 15 / 20 \mathrm{kV} / \mathrm{cm}\end{array}$ & $\begin{array}{l}\text { PEF applied to } \\
\text { lyophilized and } \\
\text { milled pomace, } \\
\text { followed by SLE } \\
\text { extraction with } \\
\text { acidulated ethanol or } \\
\text { acidulated methanol }\end{array}$ & $\begin{array}{c}\text { Optimum conditions in } \\
\text { brackets: } \\
\text { TPC: } 10.52 \mathrm{mg} \mathrm{GAE} / \mathrm{g}(\mathrm{dm}) \\
\text { (Ethanol, } 20 \mathrm{kV} / \mathrm{cm}, \\
100 \text { pulses) } \\
\text { TAC: } 1757.32 \mathrm{\mu g} / \mathrm{g} \\
\text { (Methanol, } 20 \mathrm{kV} / \mathrm{cm}, \\
100 \text { pulses) } \\
\text { Phenolic acids: } \\
625.47 \mu \mathrm{g} / \mathrm{g} \text { (Ethanol, } \\
20 \mathrm{kV} / \mathrm{cm}, 100 \mathrm{pulses}) \\
\text { Flavanols: } 297.86 \mu \mathrm{g} / \mathrm{g} \\
\text { (Methanol, } 20 \mathrm{kV} / \mathrm{cm}, \\
100 \text { pulses) } \\
\text { Flavonols: } 157.54 \mu \mathrm{gg} / \mathrm{g} \\
\text { (Ethanol, } 20 \mathrm{kV} / \mathrm{cm}, \\
100 \text { pulses) }\end{array}$ & $\begin{array}{l}\text { Optimum conditions in } \\
\text { brackets: } \\
\text { DPPH: } \\
830 \mu \mathrm{mol} \mathrm{TE} / \mathrm{g}(\mathrm{dm}) \\
\text { (Ethanol, } 20 \mathrm{kV} / \mathrm{cm}, \\
100 \text { pulses) }\end{array}$ & [113] \\
\hline
\end{tabular}


Table 7. Cont.

\begin{tabular}{|c|c|c|c|c|c|}
\hline Material & $\begin{array}{c}\text { PEF Conditions } \\
\text { (E-Equipment, P-Power, } \\
\text { FS-Field Strength, } \\
\text { f-Frequency, W-Pulse } \\
\text { Width, I-Energy Input, } \\
\text { S-Solvent, } \\
\text { L-L-Liquid-Liquid Ratio) }\end{array}$ & Procedure & $\begin{array}{c}\text { Antioxidant Composition } \\
*\end{array}$ & Antioxidant Activity * & Reference \\
\hline $\begin{array}{l}\text { Blueberry } \\
\text { pomace }\end{array}$ & $\begin{array}{c}\text { E: PEF system } \\
\text { Pulse duration: } 2 \mu \mathrm{s} \\
\text { S: acidic ethanol } \\
\text { L/L: } 1: 5 / 1: 6 / 1: 7 \\
\text { FS: } 15 / 20 / 25 \mathrm{kV} / \mathrm{cm} \\
\text { No of pulses: } 8 / 10 / 12 \\
\text { Flow rate: } 7 \mathrm{~mL} / \mathrm{min}\end{array}$ & $\begin{array}{l}\text { Thawed to liquid, } \\
\text { grinded in colloid } \\
\text { mill pomace was } \\
\text { treated with PEF in } \\
\text { liquid material } \\
\text { chamber }\end{array}$ & $\begin{array}{c}\text { Optimum conditions No of } \\
\text { pulses: } 10, \mathrm{FS}: 20 \mathrm{kV} / \mathrm{cm} \text {, } \\
\text { L/L: } 1: 6 \\
\text { TAC: } 223.13 \mathrm{mg} \text { C3GE } / \mathrm{L} \\
\text { (extract) }\end{array}$ & - & [122] \\
\hline
\end{tabular}

* Results are expressed as written in the bracket after first given result.

\subsection{Microwave-Assisted Extraction (MAE)}

Microwave-assisted extraction employs microwaves-non-ionizing electromagnetic waves-which cause changes in plant cell structure. The phenomena of heat and mass transfer which proceed in one direction occur in this type of extraction. The microwave energy is applied directly to material due to molecular interactions with the electromagnetic field through the conversion of electromagnetic energy to thermal energy. The heat must be then dissipated volumetrically inside the sample. These phenomena improve cell penetration and the internal and external diffusion of compounds is what finally leads to improved extraction yields [124]. The examples of microwaves applied in bioactive compound extraction processes for berry fruit wastes are presented in Table 8.

Pap et al. [125] studied the optimum conditions for MAE of anthocyanins from blackcurrant pomace. Variable values for power, time, solid-liquid ratio and the $\mathrm{pH}$ of the solvent were applied. Results have shown that the highest power $(700 \mathrm{~W})$ and solid-liquid ratio (1:20) but the shortest time $(10 \mathrm{~min})$ and the lowest $\mathrm{pH}(2)$ values were the most efficient conditions for anthocyanin extraction. In a HPLC study, delphinidin-3-rutoside turned out to be the most abundant anthocyanin. Davis et al. [126] applied various types of solvents and levels of power in MAE of pectin and polyphenolic rich extracts from cranberry pomace. The highest yields of polyphenols were obtained with an alkaline extraction process with a power value of $36 \mathrm{~W} / \mathrm{g}$. When SLE and MAE used with different solvents of cranberry press residues were compared, MAE resulted in a higher yield of extraction in every variant of the experiment. Values of quercetin equivalents of powdered cranberry residues were highest for MAE with 100\% acetone used as a solvent and were significantly increased compared to water and ethanol extraction processes [127]. Klavins et al. [128] compared different methods of extraction of phenolic compounds from cranberry pomace: SLE, UAE and MAE, using ethanol and trifluoroacetic acid as a solvent mixture. The extract obtained in MAE featured the lowest anthocyanin and polyphenol contents across all the studied samples.

Impact of Processing Conditions

Power: Inconclusive results of applied microwave power impact on efficiency were observed. A higher power value applied in MAE improved the extraction of anthocyanins from blackcurrant pomace [125] and also led to lower phenolic content in extracts from cranberry pomace [126]. These results are associated with the temperature increase when extended power is applied. The efficiency of MAE increases with increasing microwave power till the optimum temperature point is reached, after which it starts to decrease while the power (and temperature) is still rising [124].

Time: The shorter the time of microwave input, the better MAE efficiency was observed [125]. This may be related to the destructive impact of microwaves and the increased 
temperature on the structure of bioactive compounds. A prolonged duration of microwave power applied to a sample can promote the degradation of antioxidants [124].

Solvent type: The solvent influence on the MAE process is similar to that described in SLE. However, the capacity of the solvent to absorb microwave energy should be taken into consideration while analysing MAE experiments. Considering the analysed studies, alkaline solvents result in higher TPCs of extracts [126], and replacing ethanol with acetone was found to improve the TPCs of extracts [127]. Acetone is a solvent polar enough to be heated by microwave energy, which results in better cell heating and improved diffusion of extracted compounds [124].

Table 8. Conditions and results of microwave-assisted extraction of bioactive compounds from berry by-products.

\begin{tabular}{|c|c|c|c|c|}
\hline Source of Waste & Pretreatment & $\begin{array}{c}\text { Extraction Procedure } \\
\text { (E-Equipment, f-Frequency, } \\
\text { T-Temperature, P-Power, } \\
\text { M-Sample Weight, } \\
\text { S-L-Solid-Liquid Ratio, t-Time, } \\
\text { S-Solvent) }\end{array}$ & Antioxidant Content * & Reference \\
\hline $\begin{array}{l}\text { Blackcurrant (Ribes } \\
\text { nigrum) pomace }\end{array}$ & $\begin{array}{l}\text { Pomace was } \\
\text { obtained from } \\
\text { enzymatically } \\
\text { treated fruits }\end{array}$ & $\begin{array}{c}\text { E: single-mode cavity resonator } \\
\text { f: } 2.45 \mathrm{GHz} \\
\text { T: } 69.7^{\circ} \mathrm{C} \\
\text { P: } 140 / 420 / 700 \mathrm{~W} \\
\text { M: } 28 \mathrm{~g} \\
\text { S-L: } 1: 10,1: 13.3,1: 20 \\
\text { t: } 10 / 20 / 30 \mathrm{~min} \\
\text { solvent } \mathrm{pH}: 2 / 4.5 / 7\end{array}$ & $\begin{array}{l}\text { In optimum conditions P: } 700 \mathrm{~W} \text {, } \\
\text { t: } 10 \mathrm{~min}, \mathrm{~S}-\mathrm{L}: 1: 20, \mathrm{pH} 2 \\
\text { TAC (HPLC): } 20.4 \mathrm{mg} / \mathrm{g}(\mathrm{fw})\end{array}$ & [125] \\
\hline $\begin{array}{l}\text { Cranberry (Vaccinium } \\
\text { macrocarpon) pomace }\end{array}$ & $\begin{array}{l}\text { Freeze-dried and } \\
\text { ground pomace }\end{array}$ & $\begin{array}{c}\text { E: microwave reactor } \\
\text { S-L: } 1: 30 \\
\text { M: } 1 \mathrm{~g} \\
\text { P: } 36 / 72 \mathrm{~W} / \mathrm{g} \text { pomace } \\
\text { t: } 4 \text { min } \\
\text { Acidic extraction } \\
\text { S: } 0.1 \mathrm{M} \mathrm{HCl} \\
\text { Alkaline extraction } \\
\text { S: } 0.15 \mathrm{M} \mathrm{NaOH} \\
\text { Sequential acidic and alkaline extraction } \\
\text { S: } 0.1 \mathrm{M} \mathrm{HCl}+0.15 \mathrm{M} \mathrm{NaOH}\end{array}$ & $\begin{array}{c}\text { Acidic extraction } \\
\text { P: } 36 \text { W/g TPC: } 3.01 \mathrm{mg} \text { GAE/g (fw) } \\
\text { P: } 72 \text { W/g TPC: } 0.92 \mathrm{mg} \text { GAE/g } \\
\text { Alkaline extraction } \\
\text { P: } 36 \text { W/g TPC: } 22.78 \mathrm{mg} \mathrm{GAE} / \mathrm{g} \\
\text { P: } 72 \mathrm{~W} / \mathrm{g} \text { TPC: } 11.79 \mathrm{mg} \mathrm{GAE/g} \\
\text { Sequential } \\
\text { P: } 36 \mathrm{~W} / \mathrm{g} \text { TPC: } 11.90 \mathrm{mg} \mathrm{GAE} / \mathrm{g} \\
\text { P: } 72 \mathrm{~W} / \mathrm{g} \text { TPC: } 11.63 \mathrm{mg} \mathrm{GAE} / \mathrm{g}\end{array}$ & [126] \\
\hline $\begin{array}{l}\text { Cranberry (Vaccinium } \\
\text { macrocarpon) pomace }\end{array}$ & $\begin{array}{l}\text { Oven-dried } \\
\left(1 \mathrm{~h} / 100^{\circ} \mathrm{C}\right) \text { press } \\
\text { cake, then ground }\end{array}$ & $\begin{array}{c}\text { E: microwave press } \\
\text { S: water/ethanol/acetone } \\
\text { M: } 3.5 \mathrm{~g} \\
\text { T: } 125^{\circ} \mathrm{C} \\
\text { t: } 10 \mathrm{~min}\end{array}$ & $\begin{array}{c}\text { Water } \\
\text { TPC: } 0.02 \mathrm{mmol} \mathrm{QE} / \mathrm{g} \text { (extract) } \\
50 \% \text { ethanol } \\
\text { TPC: } 0.12 \mathrm{mmol} \mathrm{QE} / \mathrm{g} \\
100 \% \text { ethanol } \\
\text { TPC: } 0.30 \mathrm{mmol} \mathrm{QE} / \mathrm{g} \\
50 \% \text { acetone } \\
\text { TPC: } 0.27 \mathrm{mmol} \mathrm{QE} / \mathrm{g} \\
100 \% \text { acetone } \\
\text { TPC: } 0.53 \mathrm{mmol} \mathrm{QE} / \mathrm{g}\end{array}$ & [127] \\
\hline $\begin{array}{l}\text { Cranberry (Vaccinium } \\
\text { macrocarpon) pomace }\end{array}$ & $\begin{array}{l}\text { Freeze-dried and } \\
\text { homogenized } \\
\text { pomace }\end{array}$ & $\begin{array}{c}\text { E: microwave extraction unit } \\
\text { S: } 96 \% \text { ethanol and } 0.5 \% \\
\text { trifluoroacetic acid } 50 \mathrm{~mL} \\
\text { M: } 0.5 \mathrm{~g} \\
\text { P: } 600 \mathrm{~W} \\
\text { T: } 80^{\circ} \mathrm{C} \\
\text { t: } 20 \mathrm{~min}\end{array}$ & $\begin{array}{c}\text { TAC ( } \mathrm{pH} \text { differential method): } \\
0.054 \mathrm{~g} / 100 \mathrm{~g} \text { (berry powder) } \\
\text { TPC (Folin-Ciocalteu): } 1.09 \mathrm{~g} / 100 \mathrm{~g}\end{array}$ & [128] \\
\hline
\end{tabular}

* Results are expressed as written in the bracket after first given result.

\subsection{Supercritical Fluid Extraction}

Supercritical fluid extraction (SFE) includes the use of solvents at temperatures and pressures above the critical values for temperature and pressure. These conditions exhibit both the gaseous and liquid properties of solvents. SFE is commonly performed using carbon dioxide $\left(\mathrm{CO}_{2}\right)$, as it has a low critical pressure and temperature and is considered non-toxic, non-flammable and not expensive. It is also a non-polar and hydrophobic solvent. 
This is the reason why SFE is mostly used to extract lipid fractions from plant material. SFE also provides high selectivity of extraction which may be modulated by changing the conditions of extraction [129].

Examples of supercritical fluid extraction application are given in Table 9. Campalani et al. [130] compared SFE with the SLE of lipid fractions from raspberry, blueberry, blackcurrant, blackberry and strawberry pomaces. Oil yield obtained with SFE was increased compared to SLE only in the case of raspberry and blackberry oils. The study also showed particular fatty acid yields, and despite the general value of the oil yield, applying SFE resulted in higher percentage of FAs for each extract. This proves the better selectivity of SFE towards FAs. Marić et al. [131] studied differences between cold pressing and SFE of raspberry seeds. A higher oil yield was reached when cold pressing was applied, and a lower tocopherol content was observed in oil obtained with SFE. Applying SFE, Milala et al. [132] extracted oils from raspberry, chokeberry and strawberry by-products. Oil yields ranged from $12 \%$ for raspberry to $18 \%$ for strawberry pomaces. The lipid fraction was collected at particular times of the extraction process. The properties of oil-tocopherol content and fatty acid and pigment composition-were dependent on the oil collection time. Correa et al. [133] studied different conditions of SFE of oil from blackberry seeds and a comparison between SFE and SLE methods. The difference between oil yields under optimum conditions for SFE and SLE was significant. Using propane as a solvent at a temperature of $70{ }^{\circ} \mathrm{C}$ and a pressure of $20 \mathrm{MPa}$ during SFE resulted in a $2.32 \%$ oil yield. By comparison, SLE conducted using hexane resulted in a $10.51 \%$ oil yield. Antioxidant content was also measured and extracts obtained in SFE with $\mathrm{CO}_{2}$ as a solvent under optimum conditions were characterized by higher TPC values than oils extracted traditionally. Wajs-Bonikowska et al. [88] also studied properties of blackberry seed oil extracted using the SFE method. Lipid fraction yield in the case of SFE was similar to the yield when SLE with hexane was applied. SFE resulted in oil with a lower tocopherol content compared to SLE. Despite the results summarized in the Table 9, Pavlić et al. [134], on the basis of their studies applying SFE in the process of oil isolation from raspberry seeds, concluded that higher oil yields were determined by higher pressure and $\mathrm{CO}_{2}$ flow rate.

Basegmez et al. [135] obtained polyphenol-rich extracts using SFE in their research. The optimum conditions of the process were $45 \mathrm{MPa}, 60^{\circ} \mathrm{C}$ and $120 \mathrm{~min}$, and resulted in a TPC of $24.34 \mathrm{mg} \mathrm{GAE} / \mathrm{g}$ extract.

\section{Impact of Processing Conditions}

Pressure of solvent: In studies which aim was to optimize the SFE method it may be noticed that higher oil and polyphenol rich extract yields were noted when higher (but not the highest possible) pressures were applied $[133,135]$. This is connected to the increasing solubility of compounds when pressure is increased [136].

Temperature: The higher the temperature, the greater the oil and bioactive extract yields obtained $[133,135]$. Increased temperature improves the diffusion and solubility of substances [136].

Flow rate: In the reviewed studies, oils were extracted more effectively when the flow rate was high enough to be diffusion-limited [88,130-132,135]. However, this is connected with the increased amounts of solvents used in the process [136]. 
Table 9. Conditions and results of supercritical fluid extraction of oils and bioactive compounds from berry by-products.

\begin{tabular}{|c|c|c|c|c|c|}
\hline $\begin{array}{l}\text { Source of } \\
\text { Waste }\end{array}$ & $\begin{array}{l}\text { Pretreatment } \\
\text { Method }\end{array}$ & $\begin{array}{c}\text { Extraction Conditions } \\
\text { (E-Equipment, } \\
\text { M-Sample Weight, } \\
\text { S-Solvent, p-Pressure, } \\
\text { V-Volume, } \\
\text { T-Temperature, t-Time, } \\
\text { FR-Flow Rate) }\end{array}$ & Oil Yield & $\begin{array}{c}\text { Fatty Acids Profile } \\
\text { (PUFA/MUFA/SFA, } \\
\text { Dominant FA) }\end{array}$ & Reference \\
\hline $\begin{array}{l}\text { Raspberry, } \\
\text { blueberry, } \\
\text { blackcurrant, } \\
\text { blackberry, } \\
\text { strawberry } \\
\text { frozen } \\
\text { pomaces }\end{array}$ & $\begin{array}{l}\text { Air-dried with } \\
\text { water rinsing } \\
\text { (15 min water } \\
\text { rinsing, } 24 \mathrm{~h} \\
\text { drying) pomace }\end{array}$ & $\begin{array}{c}\mathrm{S}: \mathrm{CO}_{2} \\
\mathrm{M}: 3 \mathrm{~g} \\
\text { FR: } 5.0 / 2.5 \mathrm{~cm}^{3} / \mathrm{min} \\
\text { p: } 300 \mathrm{bar} \\
\mathrm{T}: 70{ }^{\circ} \mathrm{C} \\
\text { t: } 5 \mathrm{~h} \\
\text { Collected by venting into } \\
\text { hexane }\end{array}$ & $\begin{array}{c}\text { Raspberry pomace } \\
7.5 \% \\
\text { Blueberry pomace } \\
9.7 \% \\
\text { Blackcurrant pomace } \\
4.6 \% \\
\text { Blackberry pomace } \\
6.6 \% \\
\text { Strawberry pomace } \\
13.5 \%\end{array}$ & $\begin{array}{c}\text { Fatty acid yields in } \mathrm{mg} / \mathrm{g} \\
\text { Raspberry pomace } \\
\text { PUFA: } 191.0 \\
\text { MUFA: } 61.2 \\
\text { SFA: } 50.7 \\
\text { Dominant: C18:2 } \\
\text { Blueberry pomace } \\
\text { PUFA: } 134.9 \\
\text { MUFA: } 74.2 \\
\text { SFA: } 25.6 \\
\text { Dominant: C18:2 } \\
\text { Blackcurrant pomace } \\
\text { PUFA: } 60.3 \\
\text { MUFA: } 0.0 \\
\text { SFA: } 104.3 \\
\text { Dominant: C18:2 } \\
\text { Blackberry pomace } \\
\text { PUFA: } 197.0 \\
\text { MUFA: } 66.3 \\
\text { SFA: } 31.4 \\
\text { Dominant: C18:2 } \\
\text { Wild strawberry pomace } \\
\text { PUFA: } 145.8 \\
\text { MUFA: } 64.0 \\
\text { SFA: } 46.8 \\
\text { Dominant: C18:2 }\end{array}$ & [130] \\
\hline $\begin{array}{c}\text { Raspberry } \\
\text { (Rubus idaeus) } \\
\text { cv. Willamette } \\
\text { seeds }\end{array}$ & Milled seeds & $\begin{array}{c}\text { E: high pressure extraction } \\
\text { plant } \\
\mathrm{S}: \mathrm{CO}_{2} \\
\mathrm{M}: 70 \mathrm{~g} \\
\mathrm{P}: 300 \mathrm{bar} \\
\mathrm{T}: 40^{\circ} \mathrm{C} \\
\text { FR: } 0.194 \mathrm{~kg} / \mathrm{h} \\
\text { t: } 3 \mathrm{~h}\end{array}$ & $8.82 \%$ & $\begin{array}{c}\text { PUFA: } 77.90 \\
\text { MUFA: } 14.47 \\
\text { SFA: } 6.20 \\
\text { Dominant: } C 18: 2 n 6\end{array}$ & [131] \\
\hline $\begin{array}{c}\text { Raspberry } \\
\text { (Rubus idaeus } \\
\text { cv. Polka and } \\
\text { cv. Polana) } \\
\text { Chokeberry } \\
\text { (Aronia } \\
\text { melanocarpa cv. } \\
\text { Nero), } \\
\text { Strawberry } \\
\text { (Fragaria vesca } \\
\text { cv. Honeoye, } \\
\text { cv. Senga } \\
\text { Sengana and cv. } \\
\text { Polka) } \\
\text { pomaces }\end{array}$ & $\begin{array}{c}\text { Pomace dried } \\
\text { convectively in } \\
\text { industrial vacuum } \\
\text { dryers at } 70{ }^{\circ} \mathrm{C} \text { for } \\
8 \mathrm{~h} \text {. Seeds } \\
\text { separated in } \\
\text { industrial sieving } \\
\text { machines, then } \\
\text { crushed in mill } \\
\text { crusher and sieved } \\
\text { again under } \mathrm{CO}_{2} \\
\text { or nitrogen } \\
\text { atmosphere }\end{array}$ & $\begin{array}{c}\text { E: plant for extraction } \\
\text { S: } \mathrm{CO}_{2} \\
\text { M: } 14.2 \mathrm{~kg} \text { strawberry, } \\
14.5 \mathrm{~kg} \text { chokeberry, } \\
13.3 \mathrm{~kg} \text { raspberry } \\
\text { p: } 250 \text { bar, one step } \\
\text { separation at } 53 \mathrm{bar} \\
\mathrm{T}: 40^{\circ} \mathrm{C} \\
\text { FR: } 200 \mathrm{~kg} / \mathrm{h}, \\
\text { t: } 180-225 \mathrm{~min} \\
\text { with fractionation } \\
\text { (particular collection times) }\end{array}$ & $\begin{array}{c}\text { Strawberry pomace } \\
18 \% \\
\text { Chokeberry pomace } \\
15 \% \\
\text { Raspberry pomace } \\
12 \%\end{array}$ & $\begin{array}{l}\text { Values for first } \\
\text { collection } \\
\text { (after } 15 \text { min) } \\
\text { Strawberry pomace } \\
\text { PUFA: } 78.9 \\
\text { MUFA: } 15.1 \\
\text { SFA: } 5.5 \\
\text { Dominant: C18:2 } \\
\text { Chokeberry pomace } \\
\text { PUFA: } 76.6 \\
\text { MUFA: } 16.4 \\
\text { SFA: } 5.4 \\
\text { Dominant: C18:2 } \\
\text { Raspberry pomace } \\
\text { PUFA: } 84.1 \\
\text { MUFA: } 10.8 \\
\text { SFA: } 4.8 \\
\text { Dominant: C18:2 }\end{array}$ & [132] \\
\hline
\end{tabular}


Table 9. Cont.

\begin{tabular}{|c|c|c|c|c|c|}
\hline $\begin{array}{l}\text { Source of } \\
\text { Waste }\end{array}$ & $\begin{array}{l}\text { Pretreatment } \\
\text { Method }\end{array}$ & $\begin{array}{c}\text { Extraction Conditions } \\
\text { (E-Equipment, } \\
\text { M-Sample Weight, } \\
\text { S-Solvent, p-Pressure, } \\
\text { V-Volume, } \\
\text { T-Temperature, t-Time, } \\
\text { FR-Flow Rate) }\end{array}$ & Oil Yield & $\begin{array}{c}\text { Fatty Acids Profile } \\
\text { (PUFA/MUFA/SFA, } \\
\text { Dominant FA) }\end{array}$ & Reference \\
\hline $\begin{array}{c}\text { Blackberry } \\
\text { (Rubus ssp. cv. } \\
\text { Xavante) seeds }\end{array}$ & $\begin{array}{l}\text { Seeds dried in air } \\
\text { circulation oven } \\
\left(40^{\circ} \mathrm{C} / 48 \mathrm{~h}\right) \\
\text { milled, classified } \\
\text { according to } \\
\text { particle size using } \\
\text { vibratory sieve } \\
\text { shaker }\end{array}$ & $\begin{array}{c}\text { E: special apparatus } \\
\mathrm{M}: 30 \mathrm{~g} \\
\mathrm{~S}: \mathrm{CO}_{2} \text { and propane } \\
\text { p: ranging } 15.0-25.0 \mathrm{MPa} \\
\text { when using } \mathrm{CO}_{2} \text { and } \\
10.0-20.0 \mathrm{MPa} \text { when } \\
\text { using propane } \\
\mathrm{T}: 40.0-70.0^{\circ} \mathrm{C} \text { for } \mathrm{CO}_{2} \text { and } \\
30.0-70.0^{\circ} \mathrm{C} \text { for propane } \\
\text { t: for } \mathrm{CO}_{2}: 150 \mathrm{~min}, \\
\text { propane: } 60 \mathrm{~min}\end{array}$ & $\begin{array}{c}\text { Optimum } \\
\text { conditions in } \\
\text { brackets } \\
\text { S: } \mathrm{CO}_{2}\left(70{ }^{\circ} \mathrm{C},\right. \\
25 \mathrm{MPa}): 1.89 \% \\
\mathrm{~S}: \text { propane }\left(70{ }^{\circ} \mathrm{C},\right. \\
20 \mathrm{MPa}): 2.32 \%\end{array}$ & - & [133] \\
\hline $\begin{array}{l}\text { Blackberry } \\
\text { (Rubus } \\
\text { fruticosus) } \\
\text { pomace }\end{array}$ & $\begin{array}{l}\text { Pomace dried in } \\
\text { the sun and } \\
\text { crushed four times } \\
\text { in a cylinder mill }\end{array}$ & $\begin{array}{c}\text { E: plant scale fluid extractor } \\
\text { S: } \mathrm{CO}_{2} \\
\text { M: } 3500 \mathrm{~g} \\
\text { P: } 300 \mathrm{bar} \\
\text { T: } 50{ }^{\circ} \mathrm{C} \\
\text { FR: } 80 \mathrm{~kg} / \mathrm{h} \\
\text { t: } 150 \mathrm{~min}\end{array}$ & $11.4 \%$ & $\begin{array}{c}\text { FA expressed as } \\
\text { g/ } 100 \mathrm{~g} \\
\text { PUFA: } 58.2 \\
\text { MUFA: } 12.6 \\
\text { SFA: } 7.2\end{array}$ & [88] \\
\hline \multicolumn{6}{|c|}{ Polyphenol-rich extracts } \\
\hline $\begin{array}{l}\text { Source of } \\
\text { waste }\end{array}$ & Pretreatment & Extraction procedure & $\begin{array}{l}\text { Antioxidant } \\
\text { composition }\end{array}$ & Antioxidant activity * & Reference \\
\hline $\begin{array}{l}\text { Blackcurrant } \\
\text { (Ribes nigrum) } \\
\text { pomace }\end{array}$ & $\begin{array}{l}\text { Lyophilized, } \\
\text { ground pomace }\end{array}$ & $\begin{array}{l}\text { E: SFE system } \\
\text { M: } 15 \mathrm{~g} \\
\text { p: } 30-55 \mathrm{MPa} \\
\text { T: } 30-60{ }^{\circ} \mathrm{C} \\
\text { t: } 60-150 \mathrm{~min} \\
\text { FR: } 3.6 \mathrm{~g} / \mathrm{min}\end{array}$ & $\begin{array}{c}\text { Optimum } \\
\text { conditions: } \\
45 \mathrm{MPa}, 60^{\circ} \mathrm{C}, \\
120 \mathrm{~min} \\
\text { TPC: } \\
24.34 \mathrm{mg} \mathrm{GAE} / \mathrm{g} \\
\text { extract }\end{array}$ & $\begin{array}{c}\text { DPPH: } 1.59 \mathrm{mg} \text { TE } / \mathrm{g} \\
\text { (extract) } \\
\text { ORAC: } 11.35 \mathrm{mg} \mathrm{TE} / \mathrm{g} \\
\text { FRAP: } 25.00 \mathrm{mg} \mathrm{TE} / \mathrm{g}\end{array}$ & [135] \\
\hline
\end{tabular}

* Results are expressed as written in the bracket after first given result.

\subsection{Other Alternative Methods of Extraction}

\subsubsection{Pressurized Liquid Extraction}

Pressurized liquid extraction (PLE) is one of the pressurized approaches, next to SFE. PLE may, however, be used to extract high- and medium-polarity substances, whereas SFE results in extracts rich in non-polar compounds, as supercritical $\mathrm{CO}_{2}$ is used as a solvent. Combining those two methods has the benefit of obtaining extracts containing compounds characterized by different polarities [137]. There are studies reporting the extraction of compounds from residues after SFE- $\mathrm{CO}_{2}$. Brazdauskas et al. [138] studied PLE of compounds from black chokeberry pomace remaining after $\mathrm{SFE}-\mathrm{CO}_{2}$. Water, ethanol and formic acid mixture was used as a solvent, a constant pressure was set at $10.3 \mathrm{MPa}$ and variable conditions of temperature, ethanol and formic acid concentrations were examined. The optimum values were $165{ }^{\circ} \mathrm{C}, 46 \%$ ethanol and $1.8 \%$ formic acid, and resulted in response variables of $72.53 \%$ yield, $236.64 \mathrm{mg}$ GAE/g extract TPC (FolinCiocalteu method), $4.346 \mathrm{mmol} \mathrm{TE} / \mathrm{g}$ extract TEAC and $5.92 \mu \mathrm{g} / \mathrm{mL}$ as the EC50 value in a DPPH assay. Grunovaite et al. [139] also described PLE of chokeberry pomace residues after SFE- $\mathrm{CO}_{2}$. The procedure was performed at $70{ }^{\circ} \mathrm{C}, 10.3 \mathrm{MPa}$, using $96 \%$ ethanol or $100 \%$ acetone as a solvent and resulted in a $22.70 \%$ yield when ethanol was used and a $17.90 \%$ yield when acetone was used. Ethanol extract was characterized by a TPC of 
$89.41 \mathrm{mg} \mathrm{GAE} / \mathrm{g}$ extract in a Folin-Ciocalteu assay (all values expressed per dm basis) and antioxidant activities: $2.52 \mathrm{mmol} \mathrm{TE} / \mathrm{g}$ extract, $1.99 \mathrm{mmol} \mathrm{TE} / \mathrm{g}$ extract and $3.03 \mathrm{mmol} \mathrm{TE} / \mathrm{g}$ extract in ABTS, DPPH and ORAC assays, respectively. Pressurized acetone extraction resulted in a TPC value of $35.35 \mathrm{mg}$ GAE/g extract and antioxidant activities for ABTS, DPPH and ORAC assays amounting to $0.91 \mathrm{mmol} \mathrm{TE} / \mathrm{g}$ extract, $0.24 \mathrm{mmol} \mathrm{TE} / \mathrm{g}$ extract and $3.84 \mathrm{mmol} \mathrm{TE} / \mathrm{g}$ extract, respectively. In this study, also PLE alone, conducted with different conditions of temperature and using different solvents-hexane, methanol, water, acetone with water mixture and a methanol with water mixture-was examined. The optimum parameters of the process were: $130{ }^{\circ} \mathrm{C}$ and methanol used as a solvent. The conditions mentioned above allowed the obtention of the highest extraction yields (48.13\%) and values for TPC (410.20 mg GAE/g extract) and antioxidant activity of the extract, as measured in an ABTS (2.17 mmol TE/g extract) assay. Antioxidant activity, as determined by ORAC and DPPH, was highest when an acetone and water mixture under $130^{\circ} \mathrm{C}$ was used in extraction. It can be concluded that PLE extraction without a previous $\mathrm{SFE}-\mathrm{CO}_{2}$ procedure results in higher extraction yields. However, antioxidant activity determined in ABTS and DPPH assays assumes higher values when the residue after $\mathrm{SFE}-\mathrm{CO}_{2}$ is extracted again using PLE with ethanol as a solvent.

Kryževičūte et al. [140] used pressurized liquid extraction with 50\% ethanol of raspberry pomace remaining after SFE- $\mathrm{CO}_{2}$ extraction to obtain antioxidative compounds which were used to prevent beef burger spoilage during prolonged storage. The extraction yield was $19.3 \%$ and the obtained extracts were characterized by the following antioxidant capacities: $936 \mu \mathrm{mol} \mathrm{TE} / \mathrm{g}$ (extract) and $123 \mu \mathrm{mol} \mathrm{TE} / \mathrm{g}$ in ORAC and ABTS assays, respectively. The TPC of the extract was $208.3 \mathrm{mg}$ GAE/g. It was concluded that $1 \%$ PLE extract application helped to prevent oxidation in the burgers.

\subsubsection{Enzyme-Assisted Extraction}

Enzymes, such as cellulases, hemicellulases and pectinases, are used in the pretreatment of plant material prior to extraction processes to disintegrate the cell walls of material, resulting in enhanced penetration of solvents and increased extraction yields. Other benefits include shorter extraction times, reduced quantities of solvents and improved quality. However, the cost of enzymes and time and the limited ability of enzymes to complete cell wall disintegration along with the strict conditions for enzymes application are significant disadvantages of this extraction method [141].

For instance, enzymatic-assisted extraction (EAE) was used to obtain bioactive compounds from bilberry pomace, which was firstly defatted in an SFE- $\mathrm{CO}_{2}$ procedure. After removing the lipophilic fraction, enzymes were applied in particular conditions $(\mathrm{pH}$, volume of solution, temperature) and water-soluble fractions obtained under optimal chosen conditions ( $\mathrm{pH} 4.5,46^{\circ} \mathrm{C}, 1 \mathrm{~h}$, enzyme concentration 2 active units/g of pomace) were then examined. A water-soluble fraction obtained in SLE was used as a control sample. Values for yield, TPC, ABTS, ORAC and CUPRAC assays were higher when EAE was applied [142]. Kitryte et al. [143] optimized the EAE of bioactive compounds from chokeberry pomace using cellulolytic and xylanolytic enzymes. Variable values of E-S (enzyme-solid) ratio, temperature, $\mathrm{pH}$ and extraction time were applied. The optimal parameters were defined as: $\mathrm{E} / \mathrm{S} 6 \%$, temperature $40^{\circ} \mathrm{C}, \mathrm{pH} 3.5$ and $7 \mathrm{~h}$ of extraction time. The influence of the type of enzyme on polyphenol and oil extraction yields from raspberry pomace was studied by Saad et al. [144]. The enzyme that improved extraction efficiency was alkaline protease. Using that enzyme, the optimization of EAE was conducted by the authors and optimal conditions were chosen: particle size of material 50-750 $\mu \mathrm{m}, \mathrm{pH} 9$, enzyme concentration of 1.2 units $/ 100 \mathrm{~g}$ pomace, temperature of $60^{\circ} \mathrm{C}, \mathrm{S}-\mathrm{L}$ of $9 \%$ and a hydrolysis time of $2 \mathrm{~h}$, which resulted in a $5.87 \mathrm{~g} / 100 \mathrm{~g}$ pomace $(\mathrm{fw})$ extraction yield.

As the studies show, the conditions of EAE should be optimized to obtain the best possible extraction yield results. Enzymes are substances sensitive to different environmental factors and their efficiency is strictly connected with them. EAE may be considered a green 
extraction method which may be helpful in reducing solvent quantities or power levels used in the process; however, it is also a costly and demanding technique.

\section{Conclusions}

The study has shown a range of possible extraction methods which are applied to extract bioactive compounds or oils from berry fruit by-products: ultrasound-assisted, pulsed electric field-assisted, microwave-assisted, supercritical fluid, pressurized liquid and enzyme-assisted methods. There are some major differences between the presented methods with respect to their usefulness. The basic issue concerns the equipment needed for the extraction procedures. Some of the devices used in, e.g., SFE or PEF-assisted methods are advanced and costly units compared to the simple-to-operate SLE apparatus. Additionally, there are some preferable solvents and S-L ratios used in extraction, depending on the chosen method. Traditional SLE involves the use of high amounts of organic solvents, although in UAE, MAE or enzyme-assisted extraction these could be replaced with water or water-organic solvent mixtures, whereas SFE requires specific supercritical-state solvents. Time consumption is another factor that differs among the considered methods. SLE takes time, up to $24 \mathrm{~h}$, and using alternative extraction methods can reduce the duration of extraction processes to a few hours or even minutes in the case of UAE or MAE. There are, also, specific, unique parameters of conditions for each extraction method which also influence the final results but which it is not possible to compare.

The conventional (solid-liquid) processing methods involve high energy and solvent consumption, which may be harmful to the environment and financially unfavorable. However, SLE is often the most effective extraction technique, considering extraction yield. Novel, alternative extraction methods are beneficial due to their high selectivity. They are also classified as 'green' extraction methods, which means they are environment-saving. The alternative extractions conditions are still being modified to obtain the most efficient model of extraction technique and further research in this area should be carried out.

Author Contributions: Conceptualization, I.P., A.W. and A.G.; methodology, I.P., A.W. and A.G.; investigation, I.P.; resources, I.P.; data curation, I.P.; writing — original draft preparation, I.P.; writingreview and editing, A.W. and A.G.; visualization, I.P. and A.W.; supervision, A.W. and A.G.; project administration, I.P., A.W. and A.G.; funding acquisition, A.G. All authors have read and agreed to the published version of the manuscript.

Funding: This research received no external funding.

Institutional Review Board Statement: Not applicable.

Informed Consent Statement: Not applicable.

Data Availability Statement: Not applicable.

Conflicts of Interest: The authors declare no conflict of interest.

\section{Abbreviations}

$\begin{array}{ll}\text { AA } & \text { Antioxidant activity } \\ \text { C3GE } & \text { Cyanidin-3-glucoside equivalent } \\ \text { CAE } & \text { Caffeic acid equivalent } \\ \text { CUPRAC } & \text { Cupric reducing antioxidant capacity } \\ \text { DF } & \text { Dietary fiber } \\ \text { dm } & \text { Dry mass } \\ \text { EAE } & \text { Enzyme-assisted extraction } \\ \text { EE } & \text { Epicatechin equivalent } \\ \text { FA } & \text { Fatty acid } \\ \text { FAO } & \text { Food and Agriculture Organization of the United Nations } \\ \text { FE } & \text { Iron }\left(\text { Fe }{ }^{2+} \text { ) equivalent }\right. \\ \text { FRAP } & \text { Ferric reducing antioxidant power } \\ \text { fw } & \text { Fresh weight }\end{array}$




$\begin{array}{ll}\text { GAE } & \text { Gallic acid equivalent } \\ \text { MAE } & \text { Microwave-assisted extraction } \\ \text { ME } & \text { Malvidin equivalent } \\ \text { MUFA } & \text { Monounsaturated fatty acid } \\ \text { ORAC } & \text { Oxygen radical absorbance capacity } \\ \text { PEF } & \text { Pulsed electric field } \\ \text { PLE } & \text { Pressurized liquid extraction } \\ \text { PUFA } & \text { Polyunsaturated fatty acid } \\ \text { QE } & \text { Quercetin equivalent } \\ \text { RE } & \text { Rutin equivalent } \\ \text { RSM } & \text { Response-surface methodology } \\ \text { SFA } & \text { Saturated fatty acid } \\ \text { SFE } & \text { Supercritical fluid extraction } \\ \text { SLE } & \text { Solid-liquid extraction } \\ \text { T3 } & \text { Tocotrienol } \\ \text { TAC } & \text { Total anthocyanin content } \\ \text { TE } & \text { Trolox equivalent } \\ \text { TEAC } & \text { Trolox equivalent antioxidant capacity } \\ \text { TFC } & \text { Total flavonoid content } \\ \text { TP } & \text { Tocopherol } \\ \text { TPC } & \text { Total polyphenolic content } \\ \text { UAE } & \text { Ultrasound-assisted extraction } \\ \text { WHO } & \text { World Health Organization }\end{array}$

\section{References}

1. FAO FAOSTAT Crop Statistics. Available online: http://www.fao.org/faostat/en/\#data/QC (accessed on 1 March 2021).

2. Fruits and Derived Products. Available online: http://www.fao.org/es/faodef/fdef08e.htm (accessed on 6 May 2021).

3. Food Loss and Waste Facts. Available online: http://www.fao.org/resources/infographics/infographics-details/en/c/317265 (accessed on 6 May 2021).

4. FAO. Moving Forward on Food Loss and Waste Reduction; Food and Agriculture Organization of The United Nations: Rome, Italy, 2019.

5. Campos, D.A.; Gómez-García, R.; Vilas-Boas, A.A.; Madureira, A.R.; Pintado, M.M. Management of Fruit Industrial By-ProductsA Case Study on Circular Economy Approach. Molecules 2020, 25, 320. [CrossRef]

6. Schmid, V.; Steck, J.; Mayer-Miebach, E.; Behsnilian, D.; Briviba, K.; Bunzel, M.; Karbstein, H.P.; Emin, M.A. Impact of defined thermomechanical treatment on the structure and content of dietary fiber and the stability and bioaccessibility of polyphenols of chokeberry (Aronia melanocarpa) pomace. Food Res. Int. 2020, 134, 109232. [CrossRef] [PubMed]

7. Reißner, A.-M.; Alhamimi, S.; Quiles, A.; Schmidt, C.; Struck, S.; Hernando, I.; Turner, C.; Rohm, H. Composition and physicochemical properties of dried berry pomace. J. Sci. Food Agric. 2019, 99, 1284-1293. [CrossRef] [PubMed]

8. McDougall, N.; Beames, R. Composition of raspberry pomace and its nutritive value for monogastric animals. Anim. Feed Sci. Technol. 1994, 45, 139-148. [CrossRef]

9. Górnaś, P.; Juhneviča-Radenkova, K.; Radenkovs, V.; Mišina, I.; Pugajeva, I.; Soliven, A.; Seglina, D. The impact of different baking conditions on the stability of the extractable polyphenols in muffins enriched by strawberry, sour cherry, raspberry or black currant pomace. LWT Food Sci. Technol. 2016, 65, 946-953. [CrossRef]

10. Han, X.; Shen, T.; Lou, H. Dietary Polyphenols and Their Biological Significance. Int. J. Mol. Sci. 2007, 8, 950-988. [CrossRef]

11. Williamson, G. The role of polyphenols in modern nutrition. Nutr. Bull. 2017, 42, 226-235. [CrossRef]

12. Somerville, V.; Bringans, C.; Braakhuis, A. Polyphenols and Performance: A Systematic Review and Meta-Analysis. Sports Med. 2017, 47, 1589-1599. [CrossRef]

13. Ma, G.; Chen, Y. Polyphenol supplementation benefits human health via gut microbiota: A systematic review via meta-analysis. J. Funct. Foods 2020, 66, 103829. [CrossRef]

14. Liu, F.; Li, D.; Wang, X.; Cui, Y.; Li, X. Polyphenols intervention is an effective strategy to ameliorate inflammatory bowel disease: A systematic review and meta-analysis. Int. J. Food Sci. Nutr. 2020, 72, 14-25. [CrossRef] [PubMed]

15. Ammar, A.; Trabelsi, K.; Boukhris, O.; Bouaziz, B.; Müller, P.; Glenn, J.M.; Bott, N.T.; Müller, N.; Chtourou, H.; Driss, T.; et al. Effects of Polyphenol-Rich Interventions on Cognition and Brain Health in Healthy Young and Middle-Aged Adults: Systematic Review and Meta-Analysis. J. Clin. Med. 2020, 9, 1598. [CrossRef] [PubMed]

16. Shah, K.; Shah, P. Effect of Anthocyanin Supplementations on Lipid Profile and Inflammatory Markers: A Systematic Review and Meta-Analysis of Randomized Controlled Trials. Cholest 2018, 2018, 8450793. [CrossRef] [PubMed]

17. Godos, J.; Vitale, M.; Micek, A.; Ray, S.; Martini, D.; Del Rio, D.; Riccardi, G.; Galvano, F.; Grosso, G. Dietary Polyphenol Intake, Blood Pressure, and Hypertension: A Systematic Review and Meta-Analysis of Observational Studies. Antioxidants $2019,8,152$. [CrossRef] [PubMed] 
18. Fantini, M.; Benvenuto, M.; Masuelli, L.; Frajese, G.V.; Tresoldi, I.; Modesti, A.; Bei, R. In Vitro and in Vivo Antitumoral Effects of Combinations of Polyphenols, or Polyphenols and Anticancer Drugs: Perspectives on Cancer Treatment. Int. J. Mol. Sci. 2015, 16, 9236-9282. [CrossRef] [PubMed]

19. Cao, H.; Saroglu, O.; Karadag, A.; Diaconeasa, Z.; Zoccatelli, G.; Conte-Junior, C.A.; Gonzalez-Aguilar, G.A.; Ou, J.; Bai, W.; Zamarioli, C.M.; et al. Available technologies on improving the stability of polyphenols in food processing. Food Front. 2021, 2, 109-139. [CrossRef]

20. Zeng, L.; Ma, M.; Li, C.; Luo, L. Stability of tea polyphenols solution with different $\mathrm{pH}$ at different temperatures. Int. J. Food Prop. 2017, 20, 1-18. [CrossRef]

21. Chethan, S.; Malleshi, N. Finger millet polyphenols: Optimization of extraction and the effect of pH on their stability. Food Chem. 2007, 105, 862-870. [CrossRef]

22. Teleszko, M.; Nowicka, P.; Wojdyło, A. Effect of cultivar and storage temperature on identification and stability of polyphenols in strawberry cloudy juices. J. Food Compos. Anal. 2016, 54, 10-19. [CrossRef]

23. Chen, J.; Sun, H.; Wang, Y.; Wang, S.; Tao, X.; Sun, A. Stability of Apple Polyphenols as a Function of Temperature and pH. Int. J. Food Prop. 2014, 17, 1742-1749. [CrossRef]

24. Tolić, M.-T.; Jurčević, I.L.; Krbavčić, I.P.; Marković, K.; Vahčić, N. Phenolic Content, Antioxidant Capacity and Quality of Chokeberry (Aronia melanocarpa) Products. Food Technol. Biotechnol. 2015, 53, 171-179. [CrossRef]

25. Rodríguez-Werner, M.; Winterhalter, P.; Esatbeyoglu, T. Phenolic Composition, Radical Scavenging Activity and an Approach for Authentication of Aronia melanocarpa Berries, Juice, and Pomace. J. Food Sci. 2019, 84, 1791-1798. [CrossRef] [PubMed]

26. Sójka, M.; Król, B. Composition of industrial seedless black currant pomace. Eur. Food Res. Technol. 2009, 228, 597-605. [CrossRef]

27. Jara-Palacios, M.J.; Santisteban, A.; Gordillo, B.; Hernanz, D.; Heredia, F.J.; Escudero-Gilete, M.L. Comparative study of red berry pomaces (blueberry, red raspberry, red currant and blackberry) as source of antioxidants and pigments. Eur. Food Res. Technol. 2019, 245, 1-9. [CrossRef]

28. Jazić, M.; Kukrić, Z.; Vulić, J.; Četojević-Simin, D. Polyphenolic composition, antioxidant and antiproliferative effects of wild and cultivated blackberries(Rubus fruticosus L.)pomace. Int. J. Food Sci. Technol. 2019, 54, 194-201. [CrossRef]

29. Mildner-Szkudlarz, S.; Bajerska, J.; Górnaś, P.; Seglina, D.; Pilarska, A.; Jesionowski, T. Physical and Bioactive Properties of Muffins Enriched with Raspberry and Cranberry Pomace Powder: A Promising Application of Fruit By-Products Rich in Biocompounds. Plant Foods Hum. Nutr. 2016, 71, 165-173. [CrossRef]

30. Žlabur, J. Šic; Dobričević, N.; Pliestić, S.; Galić, A.; Bilić, D.P.; Voća, S. Antioxidant Potential of Fruit Juice with Added Chokeberry Powder (Aronia melanocarpa). Molecules 2017, 22, 2158. [CrossRef] [PubMed]

31. Četojević-Simin, D.D.; Velićanski, A.S.; Cvetković, D.D.; Markov, S.L.; Ćetković, G.S.; Šaponjac, V.T.T.; Vulić, J.J.; ČanadanovićBrunet, J.M.; Djilas, S.M. Bioactivity of Meeker and Willamette raspberry (Rubus idaeus L.) pomace extracts. Food Chem. 2015, 166, 407-413. [CrossRef]

32. Halász, K.; Csóka, L. Black chokeberry (Aronia melanocarpa) pomace extract immobilized in chitosan for colorimetric $\mathrm{pH}$ indicator film application. Food Packag. Shelf Life 2018, 16, 185-193. [CrossRef]

33. Raczyk, M.; Bryś, J.; Brzezińska, R.; Ostrowska-Ligęza, E.; Wirkowska-Wojdyła, M.; Górska, A. Quality assessment of cold-pressed strawberry, raspberry and blackberry seed oils intended for cosmetic purposes. Acta Sci. Pol. Technol. Aliment. 2021, 20, 127-133. [CrossRef]

34. Yang, H.Y.; Dong, S.S.; Zhang, C.H.; Wu, W.L.; Lyu, L.F.; Li, W.L. Investigation of Tocopherol Biosynthesis in Blackberry Seeds (Rubus spp.). Russ. J. Plant Physiol. 2020, 67, 76-84. [CrossRef]

35. Ying, Q.; Wojciechowska, P.; Siger, A.; Kaczmarek, A.; Rudzińska, M. Phytochemical Content, Oxidative Stability, and Nutritional Properties of Unconventional Cold-pressed Edible Oils. J. Food Nutr. Res. 2018, 6, 476-485. [CrossRef]

36. Šavikin, K.P.; Đorđević, B.S.; Ristić, M.S.; Krivokuća-Đokić, D.; Pljevljakušić, D.S.; Vulić, T. Variation in the Fatty-Acid Content in Seeds of Various Black, Red, and White Currant Varieties. Chem. Biodivers. 2013, 10, 157-165. [CrossRef]

37. Van Hoed, V.; De Clercq, N.; Echim, C.; Andjelkovic, M.; Leber, E.; Dewettinck, K.; Verhe, R. Berry Seeds: A Source of Specialty Oils with High Content of Bioactives and Nutritional Value. J. Food Lipids 2009, 16, 33-49. [CrossRef]

38. Zlatanov, M.D. Lipid composition of Bulgarian chokeberry, black currant and rose hip seed oils. J. Sci. Food Agric. 1999, 79, 1620-1624. [CrossRef]

39. Gao, F.; Birch, J. Oxidative stability, thermal decomposition, and oxidation onset prediction of carrot, flax, hemp, and canola seed oils in relation to oil composition and positional distribution of fatty acids. Eur. J. Lipid Sci. Technol. 2015, 118, 1042-1052. [CrossRef]

40. Li, H.; Fan, Y.-W.; Li, J.; Tang, L.; Hu, J.-N.; Deng, Z.-Y. Evaluating and Predicting the Oxidative Stability of Vegetable Oils with Different Fatty Acid Compositions. J. Food Sci. 2013, 78, H633-H641. [CrossRef]

41. Kochhar, S.P.; Henry, C.J.K. Oxidative stability and shelf-life evaluation of selected culinary oils. Int. J. Food Sci. Nutr. 2009, 60, 289-296. [CrossRef]

42. Nosratpour, M.; Farhoosh, R.; Sharif, A. Quantitative Indices of the Oxidizability of Fatty Acid Compositions. Eur. J. Lipid Sci. Technol. 2017, 119, 1700203. [CrossRef]

43. FAO/WHO Food and Agriculture Organization of the United Nations. Fats and Fatty Acids in Human Nutrition; Food and Agriculture Organization of The United Nations: Rome, Italy, 2010. 
44. Dyall, S.C. Long-chain omega-3 fatty acids and the brain: A review of the independent and shared effects of EPA, DPA and DHA. Front. Aging Neurosci. 2015, 7, 52. [CrossRef]

45. Mozaffarian, D.; Wu, J.H.Y. Omega-3 Fatty Acids and Cardiovascular Disease: Effects on risk factors, molecular pathways, and clinical events. J. Am. Coll. Cardiol. 2011, 58, 2047-2067. [CrossRef]

46. Costantini, L.; Molinari, R.; Farinon, B.; Merendino, N. Impact of Omega-3 Fatty Acids on the Gut Microbiota. Int. J. Mol. Sci. 2017, 18, 2645. [CrossRef] [PubMed]

47. Hu, Y.; Hu, F.B.; Manson, J.E. Marine Omega-3 Supplementation and Cardiovascular Disease: An Updated Meta-Analysis of 13 Randomized Controlled Trials Involving 127477 Participants. J. Am. Heart Assoc. 2019, 8, e013543. [CrossRef]

48. Roche, H.M. Unsaturated Fatty Acids. Proc. Nutr. Soc. 1999, 58, 397-401.

49. Mazidi, M.; Mikhailidis, D.P.; Sattar, N.; Toth, P.P.; Judd, S.; Blaha, M.J.; Hernandez, A.V.; Penson, P.E.; Banach, M. Association of types of dietary fats and all-cause and cause-specific mortality: A prospective cohort study and meta-analysis of prospective studies with 1,164,029 participants. Clin. Nutr. 2020, 39, 3677-3686. [CrossRef]

50. Jang, H.; Park, K. Omega-3 and omega-6 polyunsaturated fatty acids and metabolic syndrome: A systematic review and meta-analysis. Clin. Nutr. 2020, 39, 765-773. [CrossRef] [PubMed]

51. Wanders, A.J.; Blom, W.; Zock, P.; Geleijnse, J.M.; A Brouwer, I.; Alssema, M. Plant-derived polyunsaturated fatty acids and markers of glucose metabolism and insulin resistance: A meta-analysis of randomized controlled feeding trials. BMJ Open Diabetes Res. Care 2019, 7, e000585. [CrossRef]

52. Das, U.N. Essential fatty acids: Biochemistry, physiology and pathology. Biotechnol. J. 2006, 1, 420-439. [CrossRef] [PubMed]

53. Tocher, D.R.; Glencross, B.D. Lipids and Fatty Acids. In Dietary Nutrients, Additives and Fish Health; Wiley: Hoboken, NJ, USA, 2015; pp. 47-94. ISBN 9781119005568.

54. Schwab, U.; Lauritzen, L.; Tholstrup, T.; Halldorsson, T.; Riserus, U.; Uusitupa, M.; Becker, W. Effect of the amount and type of dietary fat on cardiometabolic risk factors and risk of developing type 2 diabetes, cardiovascular diseases, and cancer: A systematic review. Food Nutr. Res. 2014, 58, 1-26. [CrossRef] [PubMed]

55. Bozzetto, L.; Prinster, A.; Annuzzi, G.; Costagliola, L.; Mangione, A.; Vitelli, A.; Mazzarella, R.; Longobardo, M.; Mancini, M.; Vigorito, C.; et al. Liver Fat Is Reduced by an Isoenergetic MUFA Diet in a Controlled Randomized Study in Type 2 Diabetic Patients. Diabetes Care 2012, 35, 1429-1435. [CrossRef]

56. Qian, F.; Korat, A.A.; Malik, V.; Hu, F.B. Metabolic Effects of Monounsaturated Fatty Acid-Enriched Diets Compared With Carbohydrate or Polyunsaturated Fatty Acid-Enriched Diets in Patients With Type 2 Diabetes: A Systematic Review and Meta-analysis of Randomized Controlled Trials. Diabetes Care 2016, 39, 1448-1457. [CrossRef]

57. Dulf, F.V.; Andrei, S.; Bunea, A.; Socaciu, C. Fatty acid and phytosterol contents of some Romanian wild and cultivated berry pomaces. Chem. Pap. 2012, 66, 925-934. [CrossRef]

58. Piasecka, I.; Górska, A.; Ostrowska-Ligęza, E.; Kalisz, S. The Study of Thermal Properties of Blackberry, Chokeberry and Raspberry Seeds and Oils. Appl. Sci. 2021, 11, 7704. [CrossRef]

59. Bada, J.; León-Camacho, M.; Copovi, P.; Alonso, L. Characterization of Berry and Currant Seed Oils from Asturias, Spain. Int. J. Food Prop. 2013, 17, 77-85. [CrossRef]

60. Oomah, B.; Ladet, S.; Godfrey, D.V.; Liang, J.; Girard, B. Characteristics of raspberry (Rubus idaeus L.) seed oil. Food Chem. 2000, 69, 187-193. [CrossRef]

61. Knothe, G. Fuel properties of methyl esters of borage and black currant oils containing methyl $\gamma$-linolenate. Eur. J. Lipid Sci. Technol. 2013, 115, 901-908. [CrossRef]

62. Dobson, G.; Shrestha, M.; Hilz, H.; Karjalainen, R.; McDougall, G.; Stewart, D. Lipophilic components in black currant seed and pomace extracts. Eur. J. Lipid Sci. Technol. 2011, 114, 575-582. [CrossRef]

63. Johansson, A.; Laine, T.; Linna, M.-M.; Kallio, H. Variability in oil content and fatty acid composition in wild northern currants. Eur. Food Res. Technol. 2000, 211, 277-283. [CrossRef]

64. Piskernik, S.; Vidrih, R.; Demšar, L.; Koron, D.; Rogelj, M.; Žontar, T.P. Fatty acid profiles of seeds from different Ribes species. LWT Food Sci. Technol. 2018, 98, 424-427. [CrossRef]

65. Shahidi, F.; De Camargo, A.C. Tocopherols and Tocotrienols in Common and Emerging Dietary Sources: Occurrence, Applications, and Health Benefits. Int. J. Mol. Sci. 2016, 17, 1745. [CrossRef]

66. Kamal-Eldin, A. Effect of fatty acids and tocopherols on the oxidative stability of vegetable oils. Eur. J. Lipid Sci. Technol. 2006, 108, 1051-1061. [CrossRef]

67. Wagner, K.-H.; Elmadfa, I. Effects of tocopherols and their mixtures on the oxidative stability of olive oil and linseed oil under heating. Eur. J. Lipid Sci. Technol. 2000, 102, 624-629. [CrossRef]

68. Player, M.; Kim, H.; Lee, H.; Min, D. Stability of $\alpha_{--}, \gamma-$, or $\delta$-Tocopherol during Soybean Oil Oxidation. J. Food Sci. 2006, 71, C456-C460. [CrossRef]

69. Ayyildiz, H.F.; Topkafa, M.; Kara, H.; Sherazi, S.T.H. Evaluation of Fatty Acid Composition, Tocols Profile, and Oxidative Stability of Some Fully Refined Edible Oils. Int. J. Food Prop. 2015, 18, 2064-2076. [CrossRef]

70. Górnaś, P.; Soliven, A.; Seglina, D. Seed oils recovered from industrial fruit by-products are a rich source of tocopherols and tocotrienols: Rapid separation of $\alpha / \beta / \gamma / \delta$ homologues by RP-HPLC/FLD. Eur. J. Lipid Sci. Technol. 2014, 117, 773-777. [CrossRef]

71. Schneider, C. Chemistry and biology of vitamin E. Mol. Nutr. Food Res. 2004, 49, 7-30. [CrossRef] 
72. Piironen, V.; Lindsay, D.G.; Miettinen, T.A.; Toivo, J.; Lampi, A.-M. Plant Sterols: Biosynthesis, Biological Function and Their Importance to Human Nutrition. J. Sci. Food Agric. 2000, 80, 939-966. [CrossRef]

73. Guillaume, C.; Ravetti, L.; Ray, D.L.; Johnson, J. Technological Factors Affecting Sterols in Australian Olive Oils. J. Am. Oil Chem. Soc. 2011, 89, 29-39. [CrossRef]

74. Verleyen, T.; Sosinska, U.; Ioannidou, S.; Verhe, R.; Dewettinck, K.; Huyghebaert, A.; De Greyt, W. Influence of the vegetable oil refining process on free and esterified sterols. J. Am. Oil Chem. Soc. 2002, 79, 947-953. [CrossRef]

75. Hu, Y.; Xu, J.; Huang, W.; Zhao, Y.; Li, M.; Wang, M.; Zheng, L.; Lu, B. Structure-activity relationships between sterols and their thermal stability in oil matrix. Food Chem. 2018, 258, 387-392. [CrossRef] [PubMed]

76. Deepam, L.S.A.; Sundaresan, A.; Arumughan, C. Stability of Rice Bran Oil in Terms of Oryzanol, Tocopherols, Tocotrienols and Sterols. J. Am. Oil Chem. Soc. 2010, 88, 1001-1009. [CrossRef]

77. Wang, T.; Hicks, K.B.; Moreau, R. Antioxidant activity of phytosterols, oryzanol, and other phytosterol conjugates. J. Am. Oil Chem. Soc. 2002, 79, 1201-1206. [CrossRef]

78. Chang, M.; Xu, Y.; Li, X.; Shi, F.; Liu, R.; Jin, Q.; Wang, X. Effects of stigmasterol on the thermal stability of soybean oil during heating. Eur. Food Res. Technol. 2020, 246, 1755-1763. [CrossRef]

79. Qianchun, D.; Jie, S.; Mingming, Z.; Jiqu, X.; Chuyun, W.; Qingde, H.; Qi, Z.; Pingmei, G.; Fenghong, H.; Lan, W.; et al. Thermal Stability of Rapeseed Oil Fortified with Unsaturated Fatty Acid Sterol Esters. J. Am. Oil Chem. Soc. 2014, 91, 1793-1803. [CrossRef]

80. Lin, Y.; Knol, D.; Menéndez-Carreño, M.; Baris, R.; Janssen, H.-G.; Trautwein, E.A. Oxidation of sitosterol and campesterol in foods upon cooking with liquid margarines without and with added plant sterol esters. Food Chem. 2018, 241, 387-396. [CrossRef] [PubMed]

81. AbuMweis, S.S.; Barake, R.; Jones, P.J. Plant sterols/stanols as cholesterol lowering agents: A meta-analysis of randomized controlled trials. Food Nutr. Res. 2008, 52, 1-17. [CrossRef]

82. de Jong, A.; Plat, J.; Mensink, R.P. Metabolic effects of plant sterols and stanols (Review). J. Nutr. Biochem. 2003, 14, 362-369. [CrossRef]

83. Cacace, J.; Mazza, G. Mass transfer process during extraction of phenolic compounds from milled berries. J. Food Eng. 2003, 59, 379-389. [CrossRef]

84. Brglez Mojzer, E.; Knez Hrnčič, M.; Škerget, M.; Knez, Ž.; Bren, U. Polyphenols: Extraction Methods, Antioxidative Action, Bioavailability and Anticarcinogenic Effects. Molecules 2016, 21, 901. [CrossRef]

85. Martakos, I.; Kostakis, M.; Dasenaki, M.; Pentogennis, M.; Thomaidis, N. Simultaneous Determination of Pigments, Tocopherols, and Squalene in Greek Olive Oils: A Study of the Influence of Cultivation and Oil-Production Parameters. Foods $2019,9,31$. [CrossRef] [PubMed]

86. Tir, R.; Dutta, P.C.; Badjah-Hadj-Ahmed, A.Y. Effect of the extraction solvent polarity on the sesame seeds oil composition. Eur. J. Lipid Sci. Technol. 2012, 114, 1427-1438. [CrossRef]

87. Sicaire, A.-G.; Vian, M.; Fine, F.; Joffre, F.; Carré, P.; Tostain, S.; Chemat, F. Alternative Bio-Based Solvents for Extraction of Fat and Oils: Solubility Prediction, Global Yield, Extraction Kinetics, Chemical Composition and Cost of Manufacturing. Int. J. Mol. Sci. 2015, 16, 8430-8453. [CrossRef]

88. Wajs-Bonikowska, A.; Stobiecka, A.; Bonikowski, R.; Krajewska, A.; Sikora, M.; Kula, J. A comparative study on composition and antioxidant activities of supercritical carbon dioxide, hexane and ethanol extracts from blackberry (Rubus fruticosus) growing in Poland. J. Sci. Food Agric. 2017, 97, 3576-3583. [CrossRef]

89. Dimic, E.; Vujasinovic, V.; Radocaj, O.; Pastor, O. Characteristics of blackberry and raspberry seeds and oils. Acta Period. Technol. 2012, 43, 1-9. [CrossRef]

90. Šućurović, A.; Vukelić, N.; Ignjatović, L.; Brčeski, I.; Jovanović, D. Physical-chemical characteristics and oxidative stability of oil obtained from lyophilized raspberry seed. Eur. J. Lipid Sci. Technol. 2009, 111, 1133-1141. [CrossRef]

91. Radočaj, O.; Vujasinović, V.; Dimić, E.; Basić, Z. Blackberry (Rubus fruticosus L.) and raspberry (Rubus idaeus L.) seed oils extracted from dried press pomace after longterm frozen storage of berries can be used as functional food ingredients. Eur. J. Lipid Sci. Technol. 2014, 116, 1015-1024. [CrossRef]

92. Pieszka, M.; Gogol, P.; Pietras, M.; Pieszka, M. Valuable Components of Dried Pomaces of Chokeberry, Black Currant, Strawberry, Apple and Carrot as a Source of Natural Antioxidants and Nutraceuticals in the Animal Diet. Ann. Anim. Sci. 2015, 15, 475-491. [CrossRef]

93. Puganen, A.; Kallio, H.P.; Schaich, K.M.; Suomela, J.-P.; Yang, B. Red/Green Currant and Sea Buckthorn Berry Press Residues as Potential Sources of Antioxidants for Food Use. J. Agric. Food Chem. 2018, 66, 3426-3434. [CrossRef] [PubMed]

94. Ross, K.A.; Ehret, D.; Godfrey, D.; Fukumoto, L.; Diarra, M. Characterization of Pilot Scale Processed Canadian Organic Cranberry (Vaccinium macrocarpon) and Blueberry (Vaccinium angustifolium) Juice Pressing Residues and Phenolic-Enriched Extractives. Int. J. Fruit Sci. 2016, 17, 202-232. [CrossRef]

95. Vulić, J.J.; Tumbas, V.T.; Savatović, S.M.; Djilas, S.; Ćetković, G.S.; Čanadanović-Brunet, J.M. Polyphenolic content and antioxidant activity of the four berry fruits pomace extracts. Acta Period. Technol. 2011, 42, 271-279. [CrossRef]

96. Čanadanović-Brunet, J.; Vulić, J.; Ćebović, T.; Ćetković, G.; Čanadanović, V.; Djilas, S.; Šaponjac, V.T. Phenolic Profile, Antiradical and Antitumour Evaluation of Raspberries Pomace Extract from Serbia. Iran. J. Pharm. Res. 2017, 16, 142-152.

97. Laroze, L.E.; Díaz-Reinoso, B.; Moure, A.; Zúñiga, M.E.; Domínguez, H. Extraction of antioxidants from several berries pressing wastes using conventional and supercritical solvents. Eur. Food Res. Technol. 2010, 231, 669-677. [CrossRef] 
98. Kosmala, M.; Zduńczyk, Z.; Kołodziejczyk, K.; Klimczak, E.; Juśkiewicz, J.; Zdunczyk, P. Chemical composition of polyphenols extracted from strawberry pomace and their effect on physiological properties of diets supplemented with different types of dietary fibre in rats. Eur. J. Nutr. 2013, 53, 521-532. [CrossRef] [PubMed]

99. Machado, A.P.D.F.; Pereira, A.L.D.; Barbero, G.F.; Martínez, J. Recovery of anthocyanins from residues of Rubus fruticosus, Vaccinium myrtillus and Eugenia brasiliensis by ultrasound assisted extraction, pressurized liquid extraction and their combination. Food Chem. 2017, 231, 1-10. [CrossRef]

100. Medina-Torres, N.; Ayora-Talavera, T.; Espinosa-Andrews, H.; Sánchez-Contreras, A.; Pacheco, N. Ultrasound Assisted Extraction for the Recovery of Phenolic Compounds from Vegetable Sources. Agronomy 2017, 7, 47. [CrossRef]

101. D'Alessandro, L.G.; Kriaa, K.; Nikov, I.; Dimitrov, K. Ultrasound assisted extraction of polyphenols from black chokeberry. Sep. Purif. Technol. 2012, 93, 42-47. [CrossRef]

102. Herrera, M.C.; de Castro, M.D.L. Ultrasound-assisted extraction for the analysis of phenolic compounds in strawberries. Anal. Bioanal. Chem. 2004, 379, 1106-1112. [CrossRef] [PubMed]

103. Wang, W.; Jung, J.; Tomasino, E.; Zhao, Y. Optimization of solvent and ultrasound-assisted extraction for different anthocyanin rich fruit and their effects on anthocyanin compositions. LWT Food Sci. Technol. 2016, 72, 229-238. [CrossRef]

104. Gayas, B.; Kaur, G.; Gul, K. Ultrasound-Assisted Extraction of Apricot Kernel Oil: Effects on Functional and Rheological Properties. J. Food Process Eng. 2017, 40, e1243. [CrossRef]

105. Samaram, S.; Mirhosseini, H.; Tan, C.P.; Ghazali, H.; Bordbar, S.; Serjouie, A. Optimisation of ultrasound-assisted extraction of oil from papaya seed by response surface methodology: Oil recovery, radical scavenging antioxidant activity, and oxidation stability. Food Chem. 2015, 172, 7-17. [CrossRef]

106. Samaram, S.; Mirhosseini, H.; Tan, C.P.; Ghazali, H.M. Ultrasound-Assisted Extraction (UAE) and Solvent Extraction of Papaya Seed Oil: Yield, Fatty Acid Composition and Triacylglycerol Profile. Molecules 2013, 18, 12474-12487. [CrossRef]

107. Krivokapić, S.; Vlaović, M.; Vratnica, B.D.; Perović, A.; Perović, S. Biowaste as a Potential Source of Bioactive Compounds-A Case Study of Raspberry Fruit Pomace. Foods 2021, 10, 706. [CrossRef]

108. Bamba, B.S.B.; Shi, J.; Tranchant, C.C.; Xue, S.J.; Forney, C.F.; Lim, L.-T. Influence of Extraction Conditions on Ultrasound-Assisted Recovery of Bioactive Phenolics from Blueberry Pomace and Their Antioxidant Activity. Molecules 2018, 23, 1685. [CrossRef]

109. Zafra-Rojas, Q.Y.; Cruz-Cansino, N.S.; Lira, A.Q.; Gómez-Aldapa, C.A.; Alanís-García, E.; Cervantes-Elizarrarás, A.; Güemes-Vera, N.; Ramírez-Moreno, E. Application of Ultrasound in a Closed System: Optimum Condition for Antioxidants Extraction of Blackberry (Rubus fructicosus) Residues. Molecules 2016, 21, 950. [CrossRef] [PubMed]

110. Xue, H.; Tan, J.; Li, Q.; Tang, J.; Cai, X. Ultrasound-Assisted Enzymatic Extraction of Anthocyanins from Raspberry Wine Residues: Process Optimization, Isolation, Purification, and Bioactivity Determination. Food Anal. Methods 2021, 14, 1369-1386. [CrossRef]

111. Ramić, M.; Vidović, S.; Zeković, Z.; Vladić, J.; Cvejin, A.; Pavlić, B. Modeling and optimization of ultrasound-assisted extraction of polyphenolic compounds from Aronia melanocarpa by-products from filter-tea factory. Ultrason. Sonochem. 2015, 23, 360-368. [CrossRef]

112. He, B.; Zhang, L.-L.; Yue, X.-Y.; Liang, J.; Jiang, J.; Gao, X.-L.; Yue, P.-X. Optimization of Ultrasound-Assisted Extraction of phenolic compounds and anthocyanins from blueberry (Vaccinium ashei) wine pomace. Food Chem. 2016, 204, 70-76. [CrossRef] [PubMed]

113. Lončarić, A.; Celeiro, M.; Jozinović, A.; Jelinić, J.; Kovač, T.; Jokić, S.; Babić, J.; Moslavac, T.; Zavadlav, S.; Lores, M. Green Extraction Methods for Extraction of Polyphenolic Compounds from Blueberry Pomace. Foods 2020, 9, 1521. [CrossRef]

114. Gođevac, D.; Tešević, V.; Vajs, V.; Milosavljević, S.; Stanković, M. Blackberry Seed Extracts and Isolated Polyphenolic Compounds Showing Protective Effect on Human Lymphocytes DNA. J. Food Sci. 2011, 76, C1039-C1043. [CrossRef]

115. Teng, H.; Chen, L.; Huang, Q.; Wang, J.; Lin, Q.; Liu, M.; Lee, W.Y.; Song, H. Ultrasonic-Assisted Extraction of Raspberry Seed Oil and Evaluation of Its Physicochemical Properties, Fatty Acid Compositions and Antioxidant Activities. PLoS ONE 2016, 11, e0153457. [CrossRef] [PubMed]

116. Sady, S.; Matuszak, L.; Błaszczyk, A. Optimisation of ultrasonic-assisted extraction of bioactive compounds from chokeberry pomace using response surface methodology. Acta Sci. Pol. Technol. Aliment. 2015, 18, 249-256. [CrossRef]

117. Cvetanović, A.; Švarc-Gajić, J.; Zeković, Z.; Mašković, P.; Đurović, S.; Zengin, G.; Delerue-Matos, C.; Lozano-Sánchez, J.; Jakšić, A. Chemical and biological insights on aronia stems extracts obtained by different extraction techniques: From wastes to functional products. J. Supercrit. Fluids 2017, 128, 173-181. [CrossRef]

118. Oszmiański, J.; Lachowicz, S. Effect of the Production of Dried Fruits and Juice from Chokeberry (Aronia melanocarpa L.) on the Content and Antioxidative Activity of Bioactive Compounds. Molecules 2016, 21, 1098. [CrossRef] [PubMed]

119. Kumari, B.; Tiwari, B.K.; Hossain, M.B.; Brunton, N.P.; Rai, D.K. Recent Advances on Application of Ultrasound and Pulsed Electric Field Technologies in the Extraction of Bioactives from Agro-Industrial By-products. Food Bioprocess Technol. 2018, 11, 223-241. [CrossRef]

120. Pataro, G.; Bobinaite, R.; Bobinas, Česlovas; Satkauskas, S.; Raudonis, R.; Visockis, M.; Ferrari, G.; Viskelis, P. Improving the Extraction of Juice and Anthocyanins from Blueberry Fruits and Their By-products by Application of Pulsed Electric Fields. Food Bioprocess Technol. 2017, 10, 1595-1605. [CrossRef]

121. Bobinaitè, R.; Pataro, G.; Lamanauskas, N.; Šatkauskas, S.; Viskelis, P.; Ferrari, G. Application of pulsed electric field in the production of juice and extraction of bioactive compounds from blueberry fruits and their by-products. J. Food Sci. Technol. 2015, 52, 5898-5905. [CrossRef] [PubMed] 
122. Zhou, Y.; Zhao, X.; Huang, H. Effects of Pulsed Electric Fields on Anthocyanin Extraction Yield of Blueberry Processing By-Products. J. Food Process. Preserv. 2015, 39, 1898-1904. [CrossRef]

123. Gagneten, M.; Leiva, G.; Salvatori, D.; Schebor, C.; Olaiz, N. Optimization of Pulsed Electric Field Treatment for the Extraction of Bioactive Compounds from Blackcurrant. Food Bioprocess Technol. 2019, 12, 1102-1109. [CrossRef]

124. Veggi, P.C.; Martinez, J.; Meireles, M.A.A. Fundamentals of Microwave Extraction. In Microwave-Assisted Extraction for Bioactive 15 Compounds: Theory and Practice; Food Engineering Series; Springer Science and Business Media LLC: Boston, MA, USA, 2012; pp. $15-52$.

125. Pap, N.; Beszédes, S.; Pongrácz, E.; Myllykoski, L.; Gábor, M.; Gyimes, E.; Hodúr, C.; Keiski, R.L. Microwave-Assisted Extraction of Anthocyanins from Black Currant Marc. Food Bioprocess Technol. 2013, 6, 2666-2674. [CrossRef]

126. Davis, E.J.; Andreani, E.S.; Karboune, S. Production of Extracts Composed of Pectic Oligo/Polysaccharides and Polyphenolic Compounds from Cranberry Pomace by Microwave-Assisted Extraction Process. Food Bioprocess Technol. 2021, 14, 634-649. [CrossRef]

127. Raghavan, S.; Richards, M. Comparison of solvent and microwave extracts of cranberry press cake on the inhibition of lipid oxidation in mechanically separated turkey. Food Chem. 2007, 102, 818-826. [CrossRef]

128. Klavins, L.; Kviesis, J.; Klavins, M. Comparison of Methods of Extraction of Phenolic Compounds from American Cranberry (Vaccinium macrocarpon L.) Press Residues. Agron. Res. 2017, 15, 1316-1329.

129. Wrona, O.; Rafińska, K.; Możeński, C.; Buszewski, B. Supercritical Fluid Extraction of Bioactive Compounds from Plant Materials. J. AOAC Int. 2017, 100, 1624-1635. [CrossRef] [PubMed]

130. Campalani, C.; Amadio, E.; Zanini, S.; Dall'Acqua, S.; Panozzo, M.; Ferrari, S.; De Nadai, G.; Francescato, S.; Selva, M.; Perosa, A. Supercritical CO2 as a green solvent for the circular economy: Extraction of fatty acids from fruit pomace. J. CO2 Util. 2020, 41, 101259. [CrossRef]

131. Marić, B.; Abramović, B.; Ilić, N.; Krulj, J.; Kojić, J.; Perović, J.; Bodroža-Solarov, M.; Teslić, N. Valorization of red raspberry (Rubus idaeus L.) seeds as a source of health beneficial compounds: Extraction by different methods. J. Food Process. Preserv. 2020, 44, e14744. [CrossRef]

132. Milala, J.; Grzelak-Błaszczyk, K.; Sójka, M.; Kosmala, M.; Dobrzyńska-Inger, A.; Rój, E. Changes of bioactive components in berry seed oils during supercritical CO2 extraction. J. Food Process. Preserv. 2017, 42, e13368. [CrossRef]

133. Correa, M.D.S.; Fetzer, D.L.; Hamerski, F.; Corazza, M.L.; Scheer, A.D.P.; Ribani, R.H. Pressurized extraction of high-quality blackberry (Rubus spp. Xavante cultivar) seed oils. J. Supercrit. Fluids 2021, 169, 105101. [CrossRef]

134. Pavlić, B.; Pezo, L.; Marić, B.; Tukuljac, L.P.; Zeković, Z.; Solarov, M.B.; Teslić, N. Supercritical fluid extraction of raspberry seed oil: Experiments and modelling. J. Supercrit. Fluids 2020, 157, 104687. [CrossRef]

135. Basegmez, H.I.O.; Povilaitis, D.; Kitrytė, V.; Kraujalienè, V.; Šulniūtè, V.; Alasalvar, C.; Venskutonis, P.R. Biorefining of blackcurrant pomace into high value functional ingredients using supercritical $\mathrm{CO}_{2}$, pressurized liquid and enzyme assisted extractions. J. Supercrit. Fluids 2017, 124, 10-19. [CrossRef]

136. Sapkale, G.N.; Patil, S.M.; Surwase, U.S.; Bhatbhage, P.K. Supercritical Fluid Extraction. Int. J. Chem. Sci. $2010,8,729-743$.

137. Mustafa, A.; Turner, C. Pressurized liquid extraction as a green approach in food and herbal plants extraction: A review. Anal. Chim. Acta 2011, 703, 8-18. [CrossRef] [PubMed]

138. Brazdauskas, T.; Montero, L.; Venskutonis, P.; Ibañez, E.; Herrero, M. Downstream valorization and comprehensive twodimensional liquid chromatography-based chemical characterization of bioactives from black chokeberries (Aronia melanocarpa) pomace. J. Chromatogr. A 2016, 1468, 126-135. [CrossRef] [PubMed]

139. Grunovaite, L.; Pukalskienè, M.; Pukalskas, A.; Venskutonis, P.R. Fractionation of black chokeberry pomace into functional ingredients using high pressure extraction methods and evaluation of their antioxidant capacity and chemical composition. $J$. Funct. Foods 2016, 24, 85-96. [CrossRef]

140. Kryževičūtè, N.; Jaime, I.; Diez, A.M.; Rovira, J.; Venskutonis, P.R. Effect of raspberry pomace extracts isolated by high pressure extraction on the quality and shelf-life of beef burgers. Int. J. Food Sci. Technol. 2017, 52, 1852-1861. [CrossRef]

141. Puri, M.; Sharma, D.; Barrow, C.J. Enzyme-assisted extraction of bioactives from plants. Trends Biotechnol. 2012, 30, 37-44. [CrossRef]

142. Syrpas, M.; Valanciene, E.; Augustiniene, E.; Malys, N. Valorization of Bilberry (Vaccinium myrtillus L.) Pomace by EnzymeAssisted Extraction: Process Optimization and Comparison with Conventional Solid-Liquid Extraction. Antioxidants 2021, 10, 773. [CrossRef]

143. Kitrytè, V.; Kraujalienè, V.; Šulniūtè, V.; Pukalskas, A.; Venskutonis, P.R. Chokeberry pomace valorization into food ingredients by enzyme-assisted extraction: Process optimization and product characterization. Food Bioprod. Process. 2017, 105, 36-50. [CrossRef]

144. Saad, N.; Louvet, F.; Tarrade, S.; Meudec, E.; Grenier, K.; Landolt, C.; Ouk, T.; Bressollier, P. Enzyme-Assisted Extraction of Bioactive Compounds from Raspberry (Rubus idaeus L.) Pomace. J. Food Sci. 2019, 84, 1371-1381. [CrossRef] 